\title{
Selected Hydrologic Data for and Location of MX Wells in East-Central and Southern Nevada, January 1980 Through May 1996
}

By Mary L. Tumbusch and Donald H. Schaefer

U.S. GEOLOGICAL SURVEY

Open-File Report 96-469

Prepared in cooperation with the LAS VEGAS VALLEY WATER DISTRICT

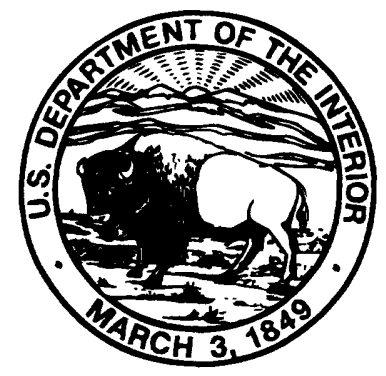

Carson City, Nevada 1996 


\section{CONTRIBUTING U.S. GEOLOGICAL SURVEY STAFF}

TECHNICAL SUPPORT

David E. Prudic, Hydrologist

\section{DATA SUPPORT}

James R. Swartwood, Hydrologic Technician Sonya L. Vasquez, Hydrologic Technician
COLLEAGUE REVIEWERS

David L. Berger, Hydrologist Michael D. Dettinger, Hydrologist

\section{GIS SUPPORT}

Rose L. Medina, Geographer

\section{PUBLICATIONS SUPPORT}

Robin L. Sweet, Technical Editor Shannon C. Demeo, Editorial Assistant

J. Christopher Stone, Scientific Illustrator 


\title{
U.S. DEPARTMENT OF THE INTERIOR BRUCE BABBITT, Secretary
}

\author{
U.S. GEOLOGICAL SURVEY \\ GORDON P. EATON, Director
}

Any use of trade names in this publication is for descriptive purposes

only and does not constitute endorsement by the U.S. Government

For additional information write to:

\section{District Chief}

U.S. Geological Survey

333 West Nye Lane, Room 203

Carson City, NV 89706-0866
Copies of this report can be purchased from:

U.S. Geological Survey

Branch of Information Services

Box 25286

Denver, CO 80225-0046 


\section{CONTENTS}

Abstract

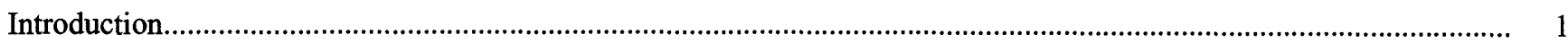

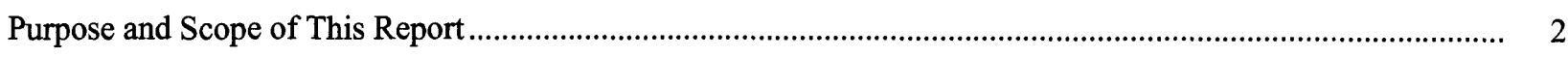

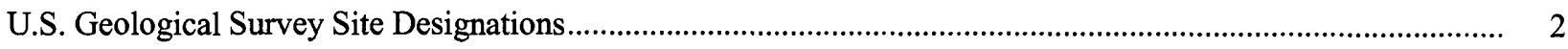

Local Well Identification ............................................................................................................... 2

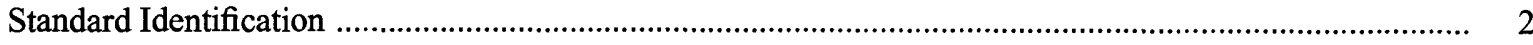

Ground-Water Data

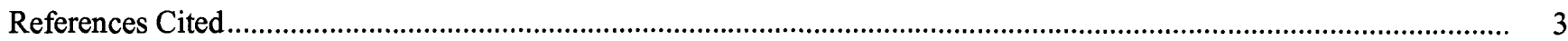

\section{PLATE}

Map showing location of MX wells and carbonate-rock province of east-central and southern Nevada

\section{TABLES}

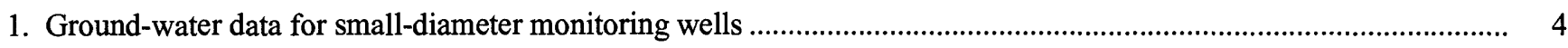

2. Ground-water data for large-diameter production and observation wells ............................................................ 23

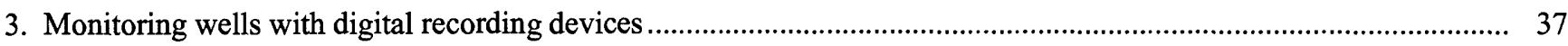

\section{CONVERSION FACTORS AND VERTICAL DATUM}

\begin{tabular}{ccl}
\hline Multiply & By & To obtain \\
\hline foot (ft) & 0.3048 & meter \\
inch (in.) & 25.4 & millimeter \\
mile (mi) & 1.609 & kilometer \\
\hline
\end{tabular}

Sea level: In this report, "sea level" refers to the National Geodetic Vertical Datum of 1929 (NGVD of 1929, formerly called "Sea-Level Datum of 1929"), which is derived from a general adjustment of the first-order leveling networks of the United States and Canada. 


\section{Selected Hydrologic Data for and Location of MX Wells in East-Central and Southern Nevada, January 1980 Through May 1996}

\author{
By Mary L. Tumbusch and Donald H. Schaefer
}

\section{ABSTRACT}

Ground-water data collected by the U.S. Geological Survey from 254 wells in east-central and southern Nevada are presented. The wells were drilled for the U.S. Air Force as part of the MX missile-siting investigation. The data have been compiled in tables and the location of wells shown on a map as part of a study by the U.S. Geological Survey, in cooperation with the Las Vegas Valley Water District.

Water level, well depth, and site status are reported from January 1980 through May 1996. Digital recording devices were installed in several of the MX wells to monitor long-term changes in local ground-water levels. Location, site information, and status of the recording devices also are included in this report.

\section{INTRODUCTION}

In 1979, the U.S. Department of the Air Force selected the Great Basin area of western Utah and eastern and southern Nevada as a suitable location for the proposed MX missile system. Extensive studies of more than 40 valleys in the Great Basin area were initiated to evaluate the availability of water resources and to determine the potential effects of the MX system on existing water uses. The principal consultant for the U.S. Air Force (Fugro National, Inc., later known as Ertec Western, Inc., Long Beach, Calif.) drilled largediameter (6- to 20-in.) production wells with nearby small-diameter (2- to 2.5-in.) observation wells at 21 sites and more than 200 small-diameter (2-in.) monitoring wells in Nevada.

On October 2, 1981, the President cancelled the MX program. A Memorandum of Agreement transferred ownership of the wells to the U.S. Geological
Survey (USGS) on June 1, 1983. Hydrologic data compiled for the MX missile-siting investigation were listed by Bunch and Harrill (1984). More recent data from the MX wells had not been widely distributed, and a map showing all the MX wells had not been published.

In 1985, the Nevada Legislature funded a program with the USGS and several other agencies to study and test the carbonate-rock aquifers of eastern and southern Nevada (U.S. Department of the Interior, 1985). One part of the study established a monitoring network to collect measurements of spring discharge, streamflow, precipitation, and ground-water levels; many of the ground-water levels were from MX wells. When the study was terminated in 1988 , an abbreviated version of the monitoring program was maintained.

In 1989, the USGS reinstated the monitoring program, in cooperation with the Las Vegas Valley Water District (LVVWD). The monitoring program was expanded to measure water levels in wells over a larger area of the carbonate-rock province in east-central and southern Nevada. Digital recording devices were installed in selected wells to monitor detailed changes in ground-water levels. Current information about the monitoring network is described in recent annual data reports published by the USGS (for example, Bauer and others, 1996, p. 573-577).

By 1990, several of the small-diameter monitoring wells originally cased with plastic pipe had been damaged by humans and animals and by exposure to the elements. Rehabilitation of the damaged wells was part of the LVVWD cooperative program. The rehabilitation included locating each damaged well, replacing the upper $3 \mathrm{ft}$ of plastic casing with galvanized steel pipe, and then recording changes in the measuring point datum with respect to land surface. 


\section{Purpose and Scope of This Report}

One purpose of this report, prepared in cooperation with the LVVWD, is to document historical ground-water levels in the MX wells. These wells represent a valuable resource for the study of groundwater levels in the carbonate-rock province. Another purpose is to locate, on a single map, all wells drilled for the U.S. Air Force MX missile-siting investigation in Nevada. The data are available from the U.S. Geological Survey data base and are now compiled in one publication.

The scope of this report includes selected hydrologic data of all wells drilled in Nevada for the U.S. Air Force during the MX missile-siting investigation.

These consist of the large-diameter production wells, with companion observation wells, and the smalldiameter monitoring wells. The coverage includes all the valleys originally investigated by the U.S. Air Force in Nevada.

\section{U.S. Geological Survey Site Designations}

Each data-collection site is assigned a unique identification on the basis of geographic location. Wells are identified by both a local (Nevada) system and by a standard "latitude-longitude" system. For convenience, a sequential number from 1 to 254 is assigned to each well symbol on plate 1 and is cross-referenced to the U.S. Geological Survey site designations in tables 1, 2, and 3 .

\section{Local Well Identification}

A local site designation is used in Nevada to identify a site by hydrographic area ${ }^{1}$ and by the official rectangular subdivision of the public lands referenced to the Mount Diablo base line and meridian. Each site designation consists of four units. The first unit is the hydrographic area number. The second unit is the township, preceded by an $\mathrm{N}$ or $\mathrm{S}$ to indicate location

\footnotetext{
${ }^{1}$ Formal hydrographic areas in Nevada were delineated systematically by the U.S. Geological Survey and Nevada Division of Water Resources in the late 1960's for scientific and administrative purposes (Rush, 1968; Cardinalli and others, 1968). The official hydrographic area names, numbers, and geographic boundaries continue to be used in U.S. Geological Survey scientific reports and Division of Water Resources administrative activities. Data are presented for 32 hydrographic areas (tables 1 and 2, pl. 1.)
}

north or south of the base line. The third unit is the range, preceded by an $\mathrm{E}$ to indicate location east of the meridian. An $\mathrm{H}$ following either the township or range number indicates a half or partial township or range. The fourth unit consists of the section number and letters designating the quarter section, quarter-quarter section, and so on (A, B, C, and D indicate the northeast, northwest, southwest, and southeast quarters, respectively), followed by a number indicating the sequence in which the site was recorded. For example, site 137A N03 E41 07CC1 is in the Tonopah Flat hydrographic area (137A). It is the first site recorded in the southwest quarter of the southwest quarter of section 7, Township 3 North, Range 41 East, Mount Diablo base line and meridian.

\section{Standard Identification}

The standard site identification is based on the grid system of latitude and longitude. The number consists of 15 digits. The first six digits denote the degrees, minutes, and seconds of latitude; the next seven digits denote the degrees, minutes, and seconds of longitude; and the last two digits (assigned sequentially) identify the sites within a 1-second grid. For example, site 390500114444901 refers to $39^{\circ} 05^{\prime} 00^{\prime \prime}$ latitude and $114^{\circ} 44^{\prime} 49^{\prime \prime}$ longitude, and it is the first site recorded in that 1 -second grid. The assigned number is retained as a permanent identifier even if a more precise latitude and longitude are later determined.

\section{GROUND-WATER DATA}

Data for the small-diameter (2-in.) monitoring wells in east-central and southern Nevada are listed in table 1 (map numbers 1-208). The table lists location and depth of well, water-level measurements, site status, and method of measurement.

Data for the large-diameter (6- to 20-in.) production wells are listed in table 2 (map numbers 209-254). Only one of the production wells listed in table 2 (well 254 ) is presently pumped for supply. Small-diameter (2- to 2.5-in.) observation wells were constructed near many of these large-diameter wells for use during aquifer tests. These observation wells are grouped with the associated large-diameter production well in the table. Location and depth of well, water-level measurements, site status, and method of measurement are listed in the table. 
The wells containing digital recording devices as part of the ongoing LVVWD monitoring program are described in table 3 . Thirteen of the 16 digital recording devices are installed in the large-diameter production wells; 2 are installed in small-diameter observation wells near a production well; and 1 is installed in a small-diameter monitoring well. Data in table 3 include location, depth of well, type of recorder, and installation date.

The locations of all MX wells in east-central and southern Nevada are shown on plate 1, with sequential numbers assigned to each well symbol. These numbers are cross-referenced to the U.S. Geological Survey site designation listed in tables 1, 2, and 3. Data listed in these tables are current as of May 1996.

\section{REFERENCES CITED}

Bauer, D.J., Foster, B.J., Joyner, J.D., and Swanson, R.A., 1996, Water resources data, Nevada, water year 1995: U.S. Geological Survey Water-Data Report NV-95-1, $734 \mathrm{p}$.
Bunch, R.L., and Harrill, J.R., 1984, Compilation of selected hydrologic data from the MX missile-siting investigation, east-central Nevada and western Utah: U.S. Geological Survey Open-File Report 84-702, 123 p.

Cardinalli, J.L., Roach, L.M., Rush, F.E., and Vasey, B.J., 1968, State of Nevada hydrographic areas: Nevada Division of Water Resources map, scale 1:500,000.

Rush, F.E., 1968, Index of hydrographic areas: Nevada Division of Water Resources, Information Report 6, $38 \mathrm{p}$.

Thomas, J.M., Mason, J.L., and Crabtree, J.D., 1986, Ground-water levels in the Great Basin region of Nevada, Utah, and adjacent states: U.S. Geological Survey Hydrologic Investigations Atlas HA-694-B, 2 sheets.

U.S. Department of the Interior, 1985, A proposed program to study the water resources of the carbonate-rock system of eastern and southern Nevada: U.S. Department of the Interior report, $14 \mathrm{p}$. 
Table 1. Ground-water data for small-diameter monitoring wells

[Site status: D, site was dry; O, obstructed; W, well destroyed; Z, other condition that would affect measured water level; --, status unknown.

Water-level method: $C$, calibrated airline; $R$, reported; S, steel tape; $T$, electric tape; --, measurement method unknown]

\begin{tabular}{|c|c|c|c|c|c|c|c|c|c|c|}
\hline \multirow[b]{2}{*}{$\begin{array}{c}\text { Map } \\
\text { no. } \\
\text { (pl. 1) }\end{array}$} & \multicolumn{2}{|c|}{ U.S. Geological Survey site designations } & \multirow[b]{2}{*}{ Latitude } & \multirow[b]{2}{*}{ Longitude } & \multirow[b]{2}{*}{$\begin{array}{c}\text { Well } \\
\text { depth } \\
\text { (feet) }\end{array}$} & \multirow{2}{*}{$\begin{array}{c}\text { Land- } \\
\text { surface } \\
\text { altitude } \\
\text { (feet above } \\
\text { sea level) }\end{array}$} & \multicolumn{4}{|c|}{ Water level } \\
\hline & Local well number & $\begin{array}{c}\text { Standard } \\
\text { identification }\end{array}$ & & & & & Date $^{1}$ & $\begin{array}{c}\text { Feet } \\
\text { below } \\
\text { land } \\
\text { surface }^{2}\end{array}$ & $\begin{array}{l}\text { Site } \\
\text { status }\end{array}$ & Method \\
\hline \multirow[t]{2}{*}{1} & \multirow[t]{2}{*}{ 137A N03 E41 07CC1 } & \multirow[t]{2}{*}{380723117252001} & \multirow[t]{2}{*}{380727} & \multirow[t]{2}{*}{1172527} & \multirow[t]{2}{*}{196} & \multirow[t]{2}{*}{4,922} & $01 / 01 / 81$ & 131 & -- & $\mathrm{R}$ \\
\hline & & & & & & & $11 / 16 / 91$ & 130.51 & $\mathrm{Z}$ & $\mathrm{S}$ \\
\hline 2 & 137A N03 E41 33AB1 & 380433117223501 & 380433 & 1172235 & 200 & 4,920 & $01 / 01 / 81$ & -- & $\mathrm{D}$ & -- \\
\hline 3 & 137A N03 E42 18CB1 & 380646117184401 & 380646 & 1171844 & 150 & 5,360 & $01 / 01 / 81$ & 140 & -- & $\mathrm{R}$ \\
\hline 4 & 137A N04 E41 04CB1 & 381345117230501 & 381345 & 1172305 & 150 & 4,881 & $01 / 01 / 81$ & 78 & -- & $\mathrm{R}$ \\
\hline 5 & 137A N05 E40 01DC1 & 381846117255001 & 381846 & 1172550 & 161 & 4,982 & $01 / 01 / 81$ & -- & $\mathrm{D}$ & -- \\
\hline 6 & 137A N07 E41 26CC1 & 382545117205201 & 382545 & 1172052 & 200 & 5,242 & $01 / 01 / 81$ & 47 & -- & $\mathrm{R}$ \\
\hline 7 & 137A N07 E42 05DC1 & 382914117170201 & 382914 & 1171702 & 150 & 5,482 & $01 / 01 / 81$ & 42 & -- & $\mathrm{R}$ \\
\hline 8 & 137A N08 E43 17BB1 & 383323117110301 & 383323 & 1171057 & 160 & 5,860 & $01 / 01 / 81$ & -- & $\mathrm{D}$ & -- \\
\hline \multirow[t]{3}{*}{9} & \multirow[t]{3}{*}{ 137A N09 E43 30AB1 } & \multirow[t]{3}{*}{383700117112401} & \multirow[t]{3}{*}{383702} & \multirow[t]{3}{*}{1171132} & \multirow[t]{3}{*}{84} & 5,742 & $01 / 01 / 81$ & 67 & -- & $\mathrm{R}$ \\
\hline & & & & & & & $05 / 15 / 84$ & 66.69 & -- & $\mathrm{S}$ \\
\hline & & & & & & & $11 / 17 / 91$ & 67.88 & -- & $\mathrm{S}$ \\
\hline 10 & 137B N11 E44 19BA1 & 384816117045201 & 384816 & 1170452 & 199 & 5,753 & $01 / 01 / 81$ & 137 & -- & $\mathrm{R}$ \\
\hline 11 & 137B N12 E43 19BA1 & 385336117112901 & 385336 & 1171129 & 200 & 5,742 & $01 / 01 / 81$ & 59 & -- & $\mathrm{R}$ \\
\hline 12 & 137B N12 E44 18AD1 & 385411117042101 & 385402 & 1170412 & 157 & 5,660 & $01 / 01 / 81$ & 113 & -- & $\mathrm{R}$ \\
\hline & & & & & & & $11 / 17 / 91$ & 60.66 & -- & $\mathrm{S}$ \\
\hline 13 & 139 N18 E50 05DA1 & 392754116213201 & 392754 & 1162132 & 167 & 6,332 & $11 / 01 / 80$ & 16 & -- & $\mathrm{R}$ \\
\hline & & & & & & & $10 / 31 / 91$ & 149.54 & -- & $\mathrm{S}$ \\
\hline 14 & 139 N18 E47 01BD1 & 392703116380401 & 392730 & 1163731 & 176 & 6,300 & $01 / 01 / 81$ & 85 & -- & $\mathrm{R}$ \\
\hline & & & & & & & $11 / 02 / 91$ & 84.30 & -- & $\mathrm{S}$ \\
\hline 15 & 139 N19 E47 28DB1 & 392849116405701 & 392849 & 1164057 & 150 & 6,275 & $02 / 01 / 81$ & 64 & -- & $\mathrm{R}$ \\
\hline 16 & 139 N19 E49 23AA1 & 393058116244501 & 393052 & 1162456 & 124 & 6,234 & $01 / 01 / 81$ & 106 & - & $\mathrm{R}$ \\
\hline & & & & & & & $10 / 31 / 91$ & 104.68 & -- & $\mathrm{S}$ \\
\hline 17 & 139 N19 E50 24AA1 & 393058116171201 & 393058 & 1161712 & 201 & 6,084 & $11 / 01 / 80$ & 35 & - & $\mathrm{R}$ \\
\hline & & & & & & & $01 / 01 / 81$ & 35 & -- & $\mathrm{R}$ \\
\hline 18 & 139 N20 E49 30BA1 & 393506116300001 & 393506 & 1163000 & 150 & 6,200 & $01 / 01 / 81$ & 86 & -- & $\mathrm{R}$ \\
\hline 19 & 139 N20 E51 13CB1 & 393654116103801 & 393630 & 1161043 & 200 & 6,365 & $02 / 01 / 81$ & 41 & -- & $\mathrm{R}$ \\
\hline 20 & 139 N21 E50 03CC1 & 394234116203701 & 394234 & 1162036 & 201 & 6,316 & $01 / 01 / 81$ & 147 & -- & $\mathrm{R}$ \\
\hline 21 & 139 N21 E50 23AA1 & 394036116183401 & 394038 & 1161842 & 139 & 6,191 & $01 / 01 / 81$ & 40 & - & $\mathrm{R}$ \\
\hline & & & & & & & $11 / 01 / 91$ & 34.00 & -- & $\mathrm{S}$ \\
\hline 22 & 139 N21 E51 24DD1 & 393957116103001 & 393956 & 1161042 & 201 & 6,316 & $01 / 01 / 81$ & 83 & -- & $\mathrm{R}$ \\
\hline 23 & 139 N22 E49 32CC1 & 394327116293901 & 394327 & 1162939 & 201 & 6,349 & $01 / 01 / 81$ & 142 & -- & $\mathrm{R}$ \\
\hline & & & & & & & $11 / 01 / 91$ & -- & $\mathrm{D}$ & -- \\
\hline 24 & 140A N14 E47 03DB1 & 390438116394301 & 390438 & 1163943 & 169 & 6,890 & $01 / 01 / 81$ & 78 & -- & $\mathrm{R}$ \\
\hline & & & & & & & $11 / 03 / 91$ & 80.06 & -- & $\mathrm{S}$ \\
\hline 25 & 140A N14 E47 21DD1 & 390150116403801 & 390154 & 1164046 & 192 & 6,956 & $01 / 01 / 81$ & 81 & -- & $\mathrm{R}$ \\
\hline & & & & & & & $11 / 03 / 91$ & 76.95 & -- & $\mathrm{S}$ \\
\hline 26 & 140A N15 E47 24CA1 & 390719116374801 & 390757 & 1163756 & 161 & 6,628 & $01 / 01 / 81$ & 60 & -- & $\mathrm{R}$ \\
\hline & & & & & & & $11 / 03 / 91$ & -- & $\mathrm{O}$ & -- \\
\hline 27 & $140 \mathrm{~A}$ N16 E47 35CB1 & 391058116385501 & 391017 & 1163900 & 182 & 6,562 & $01 / 01 / 81$ & 119 & -- & $\mathrm{R}$ \\
\hline & & & & & & & $11 / 02 / 91$ & 116.39 & -- & $\mathrm{S}$ \\
\hline 28 & $140 \mathrm{~A}$ N17 E47 27CD1 & 391809116402401 & 391621 & 1163935 & 200 & 6,464 & $01 / 01 / 81$ & 98 & -- & $\mathrm{R}$ \\
\hline 29 & 140B N09 E46 27BB1 & 380555116474901 & 383549 & 1164742 & 200 & 7,021 & $01 / 01 / 81$ & 72 & -- & $\mathrm{R}$ \\
\hline & & & & & & & $07 / 19 / 84$ & 70.12 & -- & $S$ \\
\hline & & & & & & & $11 / 04 / 91$ & -- & $\mathrm{O}$ & -- \\
\hline
\end{tabular}


Table 1. Ground-water data for small-diameter monitoring wells -Continued

\begin{tabular}{|c|c|c|c|c|c|c|c|c|c|c|}
\hline \multirow[b]{2}{*}{$\begin{array}{c}\text { Map } \\
\text { no. } \\
\text { (pl. 1) }\end{array}$} & \multicolumn{2}{|c|}{ U.S. Geological Survey site designations } & \multirow[b]{2}{*}{ Latitude } & \multirow[b]{2}{*}{ Longitude } & \multirow[b]{2}{*}{$\begin{array}{l}\text { Well } \\
\text { depth } \\
\text { (feet) }\end{array}$} & \multirow{2}{*}{$\begin{array}{c}\text { Land- } \\
\text { surface } \\
\text { altitude } \\
\text { (feet above } \\
\text { sea level) }\end{array}$} & \multicolumn{4}{|c|}{ Water level } \\
\hline & Local well number & $\begin{array}{c}\text { Standard } \\
\text { identification }\end{array}$ & & & & & Date $^{1}$ & $\begin{array}{c}\text { Feet } \\
\text { below } \\
\text { land } \\
\text { surface }^{2}\end{array}$ & $\begin{array}{l}\text { Site } \\
\text { status }\end{array}$ & Method \\
\hline 30 & 140B N09 E46 34AA1 & 383457116465301 & 383457 & 1164653 & 154 & 7,054 & $01 / 01 / 81$ & 59 & -- & $\mathbf{R}$ \\
\hline 31 & 140B N10 E46 34AA1 & 384005116480101 & 384004 & 1164656 & 136 & 7,021 & $\begin{array}{l}01 / 01 / 81 \\
07 / 19 / 84 \\
11 / 04 / 91\end{array}$ & $\begin{array}{c}101 \\
96.98 \\
100.85\end{array}$ & $\begin{array}{l}-- \\
-- \\
--\end{array}$ & $\begin{array}{l}\mathrm{R} \\
\mathrm{S} \\
\mathrm{S}\end{array}$ \\
\hline 32 & 140B N11 E46 16BD1 & 384736116481801 & 384739 & 1164822 & 154 & 6,923 & $\begin{array}{l}01 / 01 / 81 \\
11 / 03 / 91\end{array}$ & $\begin{array}{l}50 \\
53.54\end{array}$ & -- & $\begin{array}{l}\mathrm{R} \\
\mathrm{S}\end{array}$ \\
\hline 33 & 140B N12 E46 15AD1 & 385819116462301 & 385246 & 1164643 & 142 & 6,939 & $\begin{array}{l}01 / 01 / 81 \\
07 / 19 / 84 \\
11 / 03 / 91\end{array}$ & $\begin{array}{l}138 \\
138.26 \\
138.64\end{array}$ & $\begin{array}{l}-- \\
-- \\
--\end{array}$ & $\begin{array}{l}\mathrm{R} \\
\mathrm{S} \\
\mathrm{S}\end{array}$ \\
\hline 34 & 140B N12 E47 09DCl & 385306116412001 & 385306 & 1164120 & 144 & 6,890 & $\begin{array}{l}01 / 01 / 81 \\
11 / 03 / 91\end{array}$ & $\begin{array}{l}94 \\
93.49\end{array}$ & $\begin{array}{l}-- \\
--\end{array}$ & $\begin{array}{l}\mathrm{R} \\
\mathrm{S}\end{array}$ \\
\hline 35 & 140B N13 E47 07CA1 & 385836116432701 & 385836 & 1164327 & 201 & 6,990 & $01 / 01 / 81$ & -- & $\mathrm{D}$ & -- \\
\hline 36 & 141 N03 E44 06BAB1 & 380858117050701 & 380858 & 1170507 & 186 & 5,579 & $\begin{array}{l}01 / 01 / 81 \\
05 / 17 / 84\end{array}$ & $\begin{array}{l}-- \\
--\end{array}$ & $\begin{array}{l}\mathrm{D} \\
\mathrm{D}\end{array}$ & $\begin{array}{l}-- \\
--\end{array}$ \\
\hline 37 & 141 N04 E44 15CB1 & 381201117020401 & 381201 & 1170204 & 141 & 5,940 & $03 / 01 / 81$ & -- & $\mathrm{D}$ & -- \\
\hline 38 & 141 N05 E44 16BCl & 381722117030701 & 381722 & 1170307 & 143 & 6,070 & $\begin{array}{l}09 / 01 / 80 \\
01 / 01 / 81 \\
03 / 01 / 81 \\
05 / 15 / 84 \\
11 / 16 / 91\end{array}$ & $\begin{array}{l}84 \\
80 \\
80 \\
80.30 \\
79.76\end{array}$ & $\begin{array}{l}-- \\
-- \\
-- \\
-- \\
--\end{array}$ & $\begin{array}{l}\mathrm{R} \\
\mathrm{R} \\
\mathrm{R} \\
\mathrm{S} \\
\mathrm{S}\end{array}$ \\
\hline 39 & 141 N06 E44 16CCB1 & 382219117032401 & 382219 & 1170324 & 188 & 6,070 & $\begin{array}{l}01 / 01 / 81 \\
05 / 15 / 84\end{array}$ & $\begin{array}{r}185 \\
--\end{array}$ & $\overline{\mathrm{D}}$ & $\begin{array}{l}\mathbf{R} \\
--\end{array}$ \\
\hline 40 & 141 N06 E44 35DCD1 & 381935117002901 & 381935 & 1170029 & 200 & 6,005 & $\begin{array}{l}01 / 01 / 81 \\
05 / 15 / 84\end{array}$ & $\begin{array}{l}191 \\
190.49\end{array}$ & $\begin{array}{l}-- \\
--\end{array}$ & $\begin{array}{l}\mathbf{R} \\
\mathbf{S}\end{array}$ \\
\hline 41 & 142 N01 E42 18AD1 & 375626117175901 & 375626 & 1171759 & 200 & 5,020 & $01 / 01 / 81$ & -- & $\mathrm{D}$ & -- \\
\hline 42 & $142 \mathrm{~S} 01 \mathrm{E} 41$ 05CB1 & 375244117235801 & 375224 & 1172412 & 201 & 4,922 & $\begin{array}{l}01 / 01 / 81 \\
04 / 05 / 84 \\
11 / 19 / 91\end{array}$ & $\begin{array}{l}83 \\
81.87 \\
81.90\end{array}$ & $\begin{array}{l}-- \\
-- \\
--\end{array}$ & $\begin{array}{l}\mathrm{R} \\
\mathrm{S} \\
\mathrm{S}\end{array}$ \\
\hline 43 & $142 \mathrm{~S} 01 \mathrm{E} 41$ 22ABA1 & 375041117212401 & 375041 & 1172124 & 150 & 4,870 & $\begin{array}{l}01 / 01 / 81 \\
04 / 02 / 84 \\
11 / 19 / 91\end{array}$ & $\begin{array}{l}85 \\
85.85 \\
131.60\end{array}$ & $\begin{array}{l}-- \\
-- \\
--\end{array}$ & $\begin{array}{l}\mathrm{R} \\
\mathrm{S} \\
\mathrm{S}\end{array}$ \\
\hline 44 & $142 \mathrm{~S} 01 \mathrm{E} 41$ 31ACl & 374818117250701 & 374818 & 1172507 & 200 & 4,960 & $01 / 01 / 81$ & -- & $\mathrm{D}$ & -- \\
\hline 45 & 149 N01 E46 04AD1 & 375808116491801 & 375808 & 1164918 & 201 & 5,400 & $03 / 01 / 81$ & 147 & -- & $\mathbf{R}$ \\
\hline 46 & 149 N02 E47 13DB1 & 380113116394501 & 380113 & 1163945 & 189 & 5,578 & $\begin{array}{l}01 / 01 / 81 \\
02 / 01 / 81 \\
11 / 05 / 91\end{array}$ & $\begin{array}{l}86 \\
86 \\
84.37\end{array}$ & $\begin{array}{l}-- \\
-- \\
--\end{array}$ & $\begin{array}{l}\mathrm{R} \\
\mathrm{R} \\
\mathrm{S}\end{array}$ \\
\hline 47 & 149 N04 E47 12BB1 & 381310116401701 & 381302 & 1164014 & 194 & 5,906 & $\begin{array}{l}09 / 01 / 80 \\
01 / 01 / 81 \\
11 / 05 / 91\end{array}$ & $\begin{array}{l}108 \\
92 \\
93.24\end{array}$ & $\begin{array}{l}-- \\
-- \\
--\end{array}$ & $\begin{array}{l}\mathrm{R} \\
\mathrm{R} \\
\mathrm{S}\end{array}$ \\
\hline 48 & 149 N04 E48 11CD1 & 381232116344501 & 381224 & 1163433 & 154 & 5,709 & $\begin{array}{l}01 / 01 / 81 \\
11 / 05 / 91\end{array}$ & $\begin{array}{l}151 \\
150.62\end{array}$ & -- & $\begin{array}{l}\mathrm{R} \\
\mathrm{S}\end{array}$ \\
\hline 49 & 149 N05 E48 08CC1 & 381754116380601 & 381747 & 1163807 & 200 & 5,906 & $\begin{array}{l}09 / 01 / 80 \\
01 / 01 / 81 \\
03 / 01 / 81 \\
11 / 05 / 91\end{array}$ & $\begin{array}{r}73 \\
66 \\
66 \\
--\end{array}$ & $\begin{array}{c}-- \\
-- \\
-- \\
\text { W }\end{array}$ & $\begin{array}{l}\mathrm{R} \\
\mathrm{R} \\
\mathrm{R} \\
--\end{array}$ \\
\hline
\end{tabular}


Table 1. Ground-water data for small-diameter monitoring wells - Continued

\begin{tabular}{|c|c|c|c|c|c|c|c|c|c|c|c|}
\hline \multirow[b]{2}{*}{$\begin{array}{c}\text { Map } \\
\text { no. } \\
\text { (pl. 1) }\end{array}$} & \multicolumn{3}{|c|}{ U.S. Geological Survey site designations } & \multirow[b]{2}{*}{ Latitude } & \multirow[b]{2}{*}{ Longitude } & \multirow[b]{2}{*}{$\begin{array}{c}\text { Well } \\
\text { depth } \\
\text { (feet) }\end{array}$} & \multirow{2}{*}{$\begin{array}{l}\text { Land- } \\
\text { surface } \\
\text { altitude } \\
\text { (feet above } \\
\text { sea level) }\end{array}$} & \multicolumn{4}{|c|}{ Water level } \\
\hline & & Local well number & $\begin{array}{c}\text { Standard } \\
\text { identification }\end{array}$ & & & & & Date $^{1}$ & $\begin{array}{c}\text { Feet } \\
\text { below } \\
\text { land } \\
\text { surface }^{2}\end{array}$ & $\begin{array}{l}\text { Site } \\
\text { status }\end{array}$ & Method \\
\hline 50 & 151 & $\mathrm{~N} 15 \mathrm{E} 5002 \mathrm{CC} 1$ & 391114116185101 & 391144 & 1161851 & 174 & 6,464 & $\begin{array}{l}11 / 01 / 80 \\
01 / 01 / 81 \\
03 / 01 / 81 \\
10 / 30 / 91\end{array}$ & $\begin{array}{l}123 \\
123 \\
124 \\
121.28\end{array}$ & $\begin{array}{l}-- \\
-- \\
-- \\
--\end{array}$ & $\begin{array}{l}\mathrm{R} \\
\mathrm{R} \\
\mathrm{R} \\
\mathrm{S}\end{array}$ \\
\hline 51 & 151 & N16 E50 27CA1 & 391342116194401 & 391336 & 1161941 & 190 & 6,424 & $\begin{array}{l}11 / 01 / 80 \\
01 / 01 / 81 \\
03 / 01 / 81 \\
10 / 30 / 91\end{array}$ & $\begin{array}{l}108 \\
109 \\
114 \\
107.12\end{array}$ & $\begin{array}{l}-- \\
-- \\
-- \\
--\end{array}$ & $\begin{array}{l}\mathrm{R} \\
\mathrm{R} \\
\mathrm{R} \\
\mathrm{S}\end{array}$ \\
\hline 52 & 151 & N17 E50 26BC1 & 391855116191501 & 391902 & 1161903 & 160 & 6,382 & $\begin{array}{l}01 / 01 / 81 \\
10 / 30 / 91\end{array}$ & $\begin{array}{l}104 \\
125.77\end{array}$ & -- & $\begin{array}{l}\mathrm{R} \\
\mathrm{S}\end{array}$ \\
\hline 53 & 151 & N17 E51 20DD1 & 391935116144901 & 391934 & 1161440 & 186 & 6,332 & $\begin{array}{l}11 / 01 / 80 \\
01 / 01 / 81 \\
03 / 01 / 81 \\
11 / 01 / 91\end{array}$ & $\begin{array}{l}96 \\
95 \\
95 \\
94.65\end{array}$ & $\begin{array}{l}-- \\
-- \\
-- \\
--\end{array}$ & $\begin{array}{l}\mathrm{R} \\
\mathrm{R} \\
\mathrm{R} \\
\mathrm{S}\end{array}$ \\
\hline 54 & 151 & N18 E50 27BD1 & 392433116195001 & 392433 & 1161950 & 160 & 6,267 & $\begin{array}{l}11 / 01 / 80 \\
01 / 01 / 81 \\
03 / 01 / 81\end{array}$ & $\begin{array}{l}58 \\
58 \\
58\end{array}$ & $\begin{array}{l}-- \\
-- \\
--\end{array}$ & $\begin{array}{l}\mathrm{R} \\
\mathrm{R} \\
\mathrm{R}\end{array}$ \\
\hline 55 & 151 & N19 E51 33CB1 & 392847116143901 & 392847 & 1161439 & 160 & 6,234 & $\begin{array}{l}11 / 01 / 80 \\
01 / 01 / 81 \\
03 / 01 / 81 \\
10 / 31 / 91\end{array}$ & $\begin{array}{l}82 \\
83 \\
82 \\
94.32\end{array}$ & $\begin{array}{l}-- \\
-- \\
-- \\
-\end{array}$ & $\begin{array}{l}\mathrm{R} \\
\mathrm{R} \\
\mathrm{R} \\
\mathrm{S}\end{array}$ \\
\hline $\begin{array}{l}56 \\
57\end{array}$ & $\begin{array}{l}154 \\
154\end{array}$ & $\begin{array}{l}\text { N17 E55 09CC1 } \\
\text { N18 E55 16BB1 }\end{array}$ & $\begin{array}{l}392107115472701 \\
392610115474201\end{array}$ & $\begin{array}{l}392107 \\
392606\end{array}$ & $\begin{array}{l}1154727 \\
1154730\end{array}$ & $\begin{array}{l}150 \\
143\end{array}$ & $\begin{array}{l}6,050 \\
5,939\end{array}$ & $\begin{array}{l}01 / 01 / 81 \\
01 / 01 / 81 \\
03 / 01 / 81 \\
04 / 20 / 83 \\
11 / 06 / 84 \\
10 / 11 / 91\end{array}$ & $\begin{array}{l}128 \\
41 \\
41 \\
41.32 \\
40.14 \\
43.09\end{array}$ & $\begin{array}{l}-- \\
-- \\
-- \\
-- \\
-- \\
--\end{array}$ & $\begin{array}{l}\text { R } \\
R \\
\text { R } \\
\text { S } \\
\text { S } \\
\text { S }\end{array}$ \\
\hline 58 & 154 & N18 E55 33DA1 & 392301115464801 & 392301 & 1154648 & 191 & 5,971 & $\begin{array}{l}01 / 01 / 81 \\
04 / 19 / 83 \\
10 / 14 / 91\end{array}$ & $\begin{array}{l}73 \\
72.39 \\
72.57\end{array}$ & $\begin{array}{l}-- \\
-- \\
-\end{array}$ & $\begin{array}{l}\mathrm{R} \\
\mathrm{S} \\
\mathrm{S}\end{array}$ \\
\hline $\begin{array}{l}59 \\
60\end{array}$ & $\begin{array}{l}154 \\
154\end{array}$ & $\begin{array}{l}\text { N19 E55 35BB1 } \\
\text { N19 E56 13BC1 }\end{array}$ & $\begin{array}{l}392840115451001 \\
393100115372001\end{array}$ & $\begin{array}{l}392840 \\
393103\end{array}$ & $\begin{array}{l}1154510 \\
1153731\end{array}$ & $\begin{array}{l}150 \\
137\end{array}$ & $\begin{array}{l}5,890 \\
5,988\end{array}$ & $\begin{array}{l}01 / 01 / 81 \\
01 / 01 / 81 \\
10 / 11 / 91\end{array}$ & $\begin{array}{l}23 \\
51 \\
49.39\end{array}$ & $\begin{array}{l}-- \\
-- \\
--\end{array}$ & $\begin{array}{l}\mathrm{R} \\
\mathrm{R} \\
\mathrm{S}\end{array}$ \\
\hline 61 & 154 & N19 E56 25DA1 & 392903115362901 & 392903 & 1153629 & 200 & 6,040 & $01 / 01 / 81$ & 148 & -- & $\mathbf{R}$ \\
\hline $\begin{array}{l}62 \\
63\end{array}$ & $\begin{array}{l}154 \\
154\end{array}$ & $\begin{array}{l}\text { N20 E57 28CB1 } \\
\text { N21 E56 20AB1 }\end{array}$ & $\begin{array}{l}393416115340001 \\
394053115411701\end{array}$ & $\begin{array}{l}393416 \\
394053\end{array}$ & $\begin{array}{l}1153400 \\
1154117\end{array}$ & $\begin{array}{l}150 \\
135\end{array}$ & $\begin{array}{l}6,120 \\
5,906\end{array}$ & $\begin{array}{l}01 / 01 / 81 \\
01 / 01 / 81 \\
10 / 13 / 91\end{array}$ & $\begin{array}{l}100 \\
31 \\
32.96\end{array}$ & $\begin{array}{l}-- \\
-- \\
--\end{array}$ & $\begin{array}{l}\mathrm{R} \\
\mathrm{R} \\
\mathrm{S}\end{array}$ \\
\hline 64 & 154 & N22 E55 15ADCB1 & 394645115454101 & 394645 & 1154541 & 200 & 5,880 & $\begin{array}{l}01 / 01 / 81 \\
04 / 21 / 83\end{array}$ & $\begin{array}{l}23 \\
21.82\end{array}$ & - & $\begin{array}{l}\mathrm{R} \\
\mathrm{S}\end{array}$ \\
\hline 65 & 154 & N22 E56 10CA1 & 394727115391801 & 394727 & 1153918 & 139 & 5,906 & $\begin{array}{l}01 / 01 / 81 \\
03 / 01 / 81 \\
10 / 13 / 91\end{array}$ & $\begin{array}{l}25 \\
25 \\
25.65\end{array}$ & $\begin{array}{l}-- \\
-- \\
--\end{array}$ & $\begin{array}{l}\mathrm{R} \\
\mathrm{R} \\
\mathrm{S}\end{array}$ \\
\hline 66 & 154 & N23 E56 16DB1 & 395151115401001 & 395151 & 1154004 & 184 & 5,972 & $\begin{array}{l}01 / 01 / 81 \\
04 / 21 / 83 \\
10 / 14 / 91\end{array}$ & $\begin{array}{l}96 \\
96.8 \\
96.56\end{array}$ & $\begin{array}{l}-- \\
-- \\
--\end{array}$ & $\begin{array}{l}\mathrm{R} \\
\mathrm{T} \\
\mathrm{S}\end{array}$ \\
\hline
\end{tabular}


Table 1. Ground-water data for small-diameter monitoring wells - Continued

\begin{tabular}{|c|c|c|c|c|c|c|c|c|c|c|}
\hline \multirow[b]{2}{*}{$\begin{array}{l}\text { Map } \\
\text { no. } \\
\text { (pl. 1) }\end{array}$} & \multicolumn{2}{|c|}{ U.S. Geological Survey site designations } & \multirow[b]{2}{*}{ Latitude } & \multirow[b]{2}{*}{ Longitude } & \multirow[b]{2}{*}{$\begin{array}{l}\text { Well } \\
\text { depth } \\
\text { (feet) }\end{array}$} & \multirow{2}{*}{$\begin{array}{l}\text { Land- } \\
\text { surface } \\
\text { altitude } \\
\text { (feet above } \\
\text { sea level) }\end{array}$} & \multicolumn{4}{|c|}{ Water level } \\
\hline & Local well number & $\begin{array}{c}\text { Standard } \\
\text { identification }\end{array}$ & & & & & Date $^{1}$ & $\begin{array}{c}\text { Feet } \\
\text { below } \\
\text { land } \\
\text { surface }^{2}\end{array}$ & $\begin{array}{l}\text { Site } \\
\text { status }\end{array}$ & Method \\
\hline 67 & 155A N15 E54 18BD1 & 391028115560601 & 391028 & 1155606 & 160 & 6,160 & $01 / 01 / 81$ & -- & D & -- \\
\hline \multirow[t]{3}{*}{68} & 155A N16 E53 32CC1 & 384048114341001 & 391239 & 1160205 & 166 & 6,168 & $01 / 01 / 81$ & 136 & -- & $\mathrm{R}$ \\
\hline & & & & & & & $03 / 01 / 81$ & 136 & -- & $\mathrm{R}$ \\
\hline & & & & & & & $10 / 15 / 91$ & 135.70 & -- & $\mathrm{S}$ \\
\hline \multirow[t]{3}{*}{69} & 155A N17 E54 31BD1 & 391817115560701 & 391815 & 1155624 & 126 & 6,070 & $01 / 01 / 81$ & 90 & -- & $\mathbf{R}$ \\
\hline & & & & & & & $03 / 01 / 81$ & 91 & -- & $\mathrm{R}$ \\
\hline & & & & & & & $10 / 14 / 91$ & 120.56 & -- & $\mathrm{S}$ \\
\hline 70 & 155C N10 E53 28DD1 & 384122116002901 & 384122 & 1160029 & 200 & 6,280 & $01 / 01 / 81$ & -- & $\mathrm{D}$ & -- \\
\hline 71 & 156 N04 E50 09BD1 & 381321116210201 & 381321 & 1162102 & 199 & 5,460 & $03 / 01 / 81$ & -- & $\mathrm{D}$ & -- \\
\hline \multirow[t]{2}{*}{72} & 156 N04 E50 22BC1 & 381136116201101 & 381136 & 1162011 & 201 & 5,290 & $09 / 01 / 80$ & 138 & -- & $\mathbf{R}$ \\
\hline & & & & & & & $01 / 01 / 81$ & 134 & -- & $\mathbf{R}$ \\
\hline \multirow[t]{10}{*}{73} & 156 N04 E51 16CA1 & 381216116142401 & 381216 & 1161424 & 200 & 5,200 & $09 / 01 / 80$ & 73 & -- & $\mathbf{R}$ \\
\hline & & & & & & & $01 / 01 / 81$ & 55 & - & $\mathbf{R}$ \\
\hline & & & & & & & $03 / 01 / 81$ & 55 & -- & $\mathrm{R}$ \\
\hline & & & & & & & $09 / 04 / 91$ & 54.30 & -- & $\mathrm{S}$ \\
\hline & & & & & & & $04 / 06 / 93$ & 54.38 & - & $\mathrm{S}$ \\
\hline & & & & & & & $09 / 13 / 93$ & 54.36 & -- & $S$ \\
\hline & & & & & & & $01 / 05 / 94$ & 54.37 & -- & $\mathrm{S}$ \\
\hline & & & & & & & $03 / 25 / 94$ & 54.37 & - & $\mathrm{S}$ \\
\hline & & & & & & & $07 / 06 / 94$ & 54.28 & -- & $\mathrm{S}$ \\
\hline & & & & & & & $09 / 20 / 94$ & 54.41 & -- & $\mathrm{S}$ \\
\hline \multirow[t]{2}{*}{74} & 156 N04 E52 06CC1 & 381413116101401 & 381413 & 1161014 & 200 & 5,240 & $09 / 01 / 80$ & 103 & -- & $\mathrm{R}$ \\
\hline & & & & & & & $01 / 01 / 81$ & 101 & -- & $\mathrm{R}$ \\
\hline \multirow[t]{4}{*}{75} & 156 N05 E50 01BD1 & 381923116174501 & 381925 & 1161733 & 200 & 5,266 & $09 / 01 / 80$ & 113 & -- & $\mathbf{R}$ \\
\hline & & & & & & & $01 / 01 / 81$ & 115 & -- & $\mathbf{R}$ \\
\hline & & & & & & & $03 / 01 / 81$ & 114 & - & $\mathbf{R}$ \\
\hline & & & & & & & $10 / 16 / 91$ & 114.17 & -- & $\mathrm{S}$ \\
\hline \multirow[t]{16}{*}{76} & 156 N06 E51 17BD1 & 382255116153801 & 382255 & 1161538 & 188 & 5,315 & $09 / 01 / 80$ & 102 & -- & $\mathrm{R}$ \\
\hline & & & & & & & $01 / 01 / 81$ & 79 & -- & $\mathrm{R}$ \\
\hline & & & & & & & $03 / 01 / 81$ & 79 & -- & $\mathrm{R}$ \\
\hline & & & & & & & $10 / 16 / 91$ & 78.73 & -- & $\mathrm{S}$ \\
\hline & & & & & & & $10 / 21 / 91$ & 78.73 & -- & $\mathrm{S}$ \\
\hline & & & & & & & $03 / 27 / 92$ & 78.77 & -- & $\mathrm{S}$ \\
\hline & & & & & & & $06 / 25 / 92$ & 78.81 & -- & $\mathrm{S}$ \\
\hline & & & & & & & $09 / 18 / 92$ & 79.00 & -- & $\mathrm{S}$ \\
\hline & & & & & & & $12 / 09 / 92$ & 79.02 & -- & $\mathrm{S}$ \\
\hline & & & & & & & $04 / 06 / 93$ & 78.98 & - & $\mathrm{S}$ \\
\hline & & & & & & & $07 / 01 / 93$ & 78.81 & -- & $\mathrm{S}$ \\
\hline & & & & & & & $09 / 13 / 93$ & 78.87 & - & $\mathrm{S}$ \\
\hline & & & & & & & $12 / 20 / 93$ & 78.90 & - & $\mathrm{S}$ \\
\hline & & & & & & & $03 / 26 / 94$ & 78.83 & -- & $\mathrm{S}$ \\
\hline & & & & & & & 07/06/94 & 78.79 & -- & $\mathrm{S}$ \\
\hline & & & & & & & $09 / 20 / 94$ & 78.85 & -- & $\mathrm{S}$ \\
\hline \multirow[t]{2}{*}{77} & 156 N07 E51 04DC1 & 382926116141501 & 382926 & 1161415 & 200 & 5,490 & $09 / 01 / 80$ & 132 & - & $\mathbf{R}$ \\
\hline & & & & & & & $03 / 01 / 81$ & 129 & - & $\mathbf{R}$ \\
\hline 78 & 170 S01 E56 18DD1 & 375633115425201 & 375633 & 1154252 & 200 & 4,900 & $12 / 01 / 80$ & -- & $\mathrm{D}$ & -- \\
\hline 79 & 170 S01 E56 28BD1 & 375513115411301 & 375513 & 1154113 & 200 & 5,000 & $12 / 01 / 80$ & -- & $\mathrm{D}$ & -- \\
\hline
\end{tabular}


Table 1. Ground-water data for small-diameter monitoring wells - Continued

\begin{tabular}{|c|c|c|c|c|c|c|c|c|c|c|c|}
\hline \multirow[b]{2}{*}{$\begin{array}{c}\text { Map } \\
\text { no. } \\
\text { (pl. 1) }\end{array}$} & \multicolumn{3}{|c|}{ U.S. Geological Survey site designations } & \multirow[b]{2}{*}{ Latitude } & \multirow[b]{2}{*}{ Longitude } & \multirow[b]{2}{*}{$\begin{array}{c}\text { Well } \\
\text { depth } \\
\text { (feet) }\end{array}$} & \multirow{2}{*}{$\begin{array}{c}\text { Land- } \\
\text { surface } \\
\text { altitude } \\
\text { (feet above } \\
\text { sea level) }\end{array}$} & \multicolumn{4}{|c|}{ Water level } \\
\hline & & Local well number & $\begin{array}{c}\text { Standard } \\
\text { identification }\end{array}$ & & & & & Date $^{1}$ & $\begin{array}{c}\text { Feet } \\
\text { below } \\
\text { land } \\
\text { surface }^{2}\end{array}$ & $\begin{array}{c}\text { Site } \\
\text { status }\end{array}$ & Method \\
\hline \multirow[t]{12}{*}{80} & 170 & S02 E55 09AD1 & 374729115483301 & 374718 & 1154723 & 192 & 4,921 & $01 / 01 / 81$ & 171 & -- & $\mathbf{R}$ \\
\hline & & & & & & & & $03 / 01 / 81$ & 170 & -- & $\mathbf{R}$ \\
\hline & & & & & & & & $03 / 11 / 85$ & 182.03 & -- & $S$ \\
\hline & & & & & & & & $11 / 20 / 91$ & 181.92 & -- & $\mathrm{S}$ \\
\hline & & & & & & & & $03 / 25 / 92$ & 181.96 & -- & $\mathrm{S}$ \\
\hline & & & & & & & & $06 / 18 / 92$ & 181.98 & -- & $\mathrm{S}$ \\
\hline & & & & & & & & $09 / 18 / 92$ & 182.08 & -- & $\mathrm{S}$ \\
\hline & & & & & & & & $12 / 09 / 92$ & 182.11 & -- & $\mathrm{S}$ \\
\hline & & & & & & & & $01 / 05 / 94$ & 182.13 & -- & $\mathrm{S}$ \\
\hline & & & & & & & & $03 / 22 / 94$ & 178.99 & -- & $\mathrm{S}$ \\
\hline & & & & & & & & $07 / 06 / 94$ & -- & $\mathrm{O}$ & -- \\
\hline & & & & & & & & $09 / 20 / 94$ & - & $\mathrm{O}$ & -- \\
\hline \multirow[t]{10}{*}{81} & 170 & S02 E55 24CB1 & 374523115440901 & 374523 & 1154445 & 153 & 4,800 & $11 / 01 / 80$ & 55 & -- & $\mathrm{R}$ \\
\hline & & & & & & & & $01 / 01 / 81$ & 55 & -- & $\mathbf{R}$ \\
\hline & & & & & & & & $03 / 05 / 81$ & 55.31 & -- & $\mathrm{S}$ \\
\hline & & & & & & & & $03 / 11 / 85$ & 54.70 & -- & $\mathrm{S}$ \\
\hline & & & & & & & & $04 / 10 / 90$ & 54.40 & -- & $\mathrm{C}$ \\
\hline & & & & & & & & $11 / 20 / 91$ & 54.53 & - & $\mathrm{S}$ \\
\hline & & & & & & & & $04 / 12 / 93$ & 54.52 & -- & $\mathrm{S}$ \\
\hline & & & & & & & & $12 / 15 / 93$ & 54.47 & -- & $\mathrm{S}$ \\
\hline & & & & & & & & $03 / 31 / 94$ & 54.51 & -- & $\mathbf{S}$ \\
\hline & & & & & & & & $09 / 20 / 94$ & 54.54 & -- & $\mathrm{S}$ \\
\hline \multirow[t]{6}{*}{82} & 170 & S02 E56 05AD1 & 380338115422001 & 374813 & 1154149 & 193 & 4,922 & $11 / 01 / 80$ & 121 & -- & $\mathbf{R}$ \\
\hline & & & & & & & & $11 / 20 / 91$ & 120.12 & $Z$ & $S$ \\
\hline & & & & & & & & $04 / 12 / 93$ & 120.12 & -- & $\mathrm{S}$ \\
\hline & & & & & & & & $12 / 15 / 93$ & 120.11 & -- & $\mathrm{S}$ \\
\hline & & & & & & & & $03 / 31 / 94$ & 120.08 & -- & $\mathrm{S}$ \\
\hline & & & & & & & & $09 / 20 / 94$ & 120.08 & -- & $\mathrm{S}$ \\
\hline \multirow[t]{14}{*}{83} & 170 & S02 E56 32DAAA1 & 374345115414701 & 374345 & 1154147 & 189 & 4,921 & $11 / 01 / 80$ & 124 & -- & $\mathbf{R}$ \\
\hline & & & & & & & & $01 / 01 / 81$ & 124 & -- & $\mathbf{R}$ \\
\hline & & & & & & & & $03 / 01 / 81$ & 129 & - & $\mathbf{R}$ \\
\hline & & & & & & & & $03 / 05 / 81$ & 119.93 & -- & $S$ \\
\hline & & & & & & & & $03 / 11 / 85$ & 124.10 & -- & $\mathrm{S}$ \\
\hline & & & & & & & & $11 / 20 / 91$ & 113.54 & -- & $\mathrm{S}$ \\
\hline & & & & & & & & $03 / 25 / 92$ & 123.48 & - & $\mathrm{S}$ \\
\hline & & & & & & & & $06 / 17 / 92$ & 123.52 & -- & $\mathrm{S}$ \\
\hline & & & & & & & & $09 / 18 / 92$ & 123.57 & -- & $\mathrm{S}$ \\
\hline & & & & & & & & $12 / 09 / 92$ & 123.58 & -- & $\mathrm{S}$ \\
\hline & & & & & & & & $01 / 05 / 93$ & 123.55 & -- & $\mathrm{S}$ \\
\hline & & & & & & & & $03 / 22 / 94$ & 123.58 & -- & $\mathrm{S}$ \\
\hline & & & & & & & & $07 / 07 / 94$ & 123.60 & -- & $\mathrm{S}$ \\
\hline & & & & & & & & $09 / 20 / 94$ & 123.58 & -- & $\mathrm{S}$ \\
\hline 84 & 170 & S03 E54 12BCBC1 & 380829115513401 & 374212 & 1155137 & 200 & 4,975 & $01 / 01 / 81$ & -- & $\mathrm{D}$ & -- \\
\hline \multirow[t]{4}{*}{85} & 170 & S03 E55 07CC1 & 380738115502901 & 374139 & 1155028 & 148 & 4,922 & $11 / 01 / 80$ & 103 & -- & $\mathbf{R}$ \\
\hline & & & & & & & & $01 / 01 / 81$ & 104 & -- & $\mathbf{R}$ \\
\hline & & & & & & & & $03 / 11 / 85$ & 106.44 & -- & $\mathrm{S}$ \\
\hline & & & & & & & & $04 / 10 / 90$ & 111 & -- & $\mathrm{C}$ \\
\hline
\end{tabular}


Table 1. Ground-water data for small-diameter monitoring wells - Continued

\begin{tabular}{|c|c|c|c|c|c|c|c|c|c|c|c|}
\hline \multirow[b]{2}{*}{$\begin{array}{c}\text { Map } \\
\text { no. } \\
\text { (pl. 1) }\end{array}$} & \multicolumn{3}{|c|}{ U.S. Geological Survey site designations } & \multirow[b]{2}{*}{ Latitude } & \multirow[b]{2}{*}{ Longitude } & \multirow[b]{2}{*}{$\begin{array}{c}\text { Well } \\
\text { depth } \\
\text { (feet) }\end{array}$} & \multirow{2}{*}{$\begin{array}{c}\text { Land- } \\
\text { surface } \\
\text { altitude } \\
\text { (feet above } \\
\text { sea level) }\end{array}$} & \multicolumn{4}{|c|}{ Water level } \\
\hline & & Local well number & $\begin{array}{c}\text { Standard } \\
\text { identification }\end{array}$ & & & & & Date $^{1}$ & $\begin{array}{c}\text { Feet } \\
\text { below } \\
\text { land } \\
\text { surface }^{2}\end{array}$ & $\begin{array}{l}\text { Site } \\
\text { status }\end{array}$ & Method \\
\hline \multirow[t]{4}{*}{85} & 170 & S03 E55 07CC1 & 380738115502901 & 374139 & 1155028 & 148 & 4,922 & $11 / 20 / 91$ & 112.09 & -- & $\mathrm{S}$ \\
\hline & & & & & & & & $04 / 12 / 93$ & 113.50 & -- & $\mathrm{S}$ \\
\hline & & & & & & & & $12 / 15 / 93$ & -- & $\mathrm{O}$ & -- \\
\hline & & & & & & & & $03 / 31 / 94$ & -- & $\mathrm{O}$ & -- \\
\hline \multirow[t]{9}{*}{86} & 170 & S03 E55 36ADDCl & 373833115441101 & 373833 & 1154411 & 195 & 4,921 & $01 / 01 / 81$ & 140 & -- & $\mathbf{R}$ \\
\hline & & & & & & & & $03 / 05 / 81$ & 138.98 & -- & $\mathrm{S}$ \\
\hline & & & & & & & & $11 / 20 / 91$ & 144.70 & -- & $\mathrm{S}$ \\
\hline & & & & & & & & $09 / 18 / 92$ & 144.90 & -- & $S$ \\
\hline & & & & & & & & $12 / 09 / 92$ & 144.98 & -- & $\mathrm{S}$ \\
\hline & & & & & & & & $01 / 05 / 94$ & 145.25 & -- & $\mathrm{S}$ \\
\hline & & & & & & & & $03 / 22 / 94$ & 145.30 & -- & $\mathrm{S}$ \\
\hline & & & & & & & & $07 / 07 / 94$ & 145.42 & - & $\mathrm{S}$ \\
\hline & & & & & & & & $09 / 20 / 94$ & 145.48 & -- & $\mathrm{S}$ \\
\hline 87 & 171 & N01 E60 33CC1 & 375349115151501 & 375349 & 1151515 & 200 & 4,990 & $01 / 01 / 80$ & -- & $\mathrm{D}$ & -- \\
\hline 88 & 171 & N03 E59 12AA1 & 380823115173901 & 380823 & 1151739 & 200 & 5,080 & $11 / 01 / 80$ & -- & $\mathrm{D}$ & -- \\
\hline \multirow[t]{5}{*}{89} & 171 & N03 E59 12AA2 & 380823115173902 & 380823 & 1151739 & 354 & 5,087 & $08 / 27 / 93$ & 247.2 & - & $\mathrm{T}$ \\
\hline & & & & & & & & $09 / 14 / 93$ & 247.8 & -- & $\mathrm{T}$ \\
\hline & & & & & & & & $03 / 23 / 94$ & -- & $\mathrm{D}$ & -- \\
\hline & & & & & & & & $07 / 06 / 94$ & -- & $\mathrm{D}$ & -- \\
\hline & & & & & & & & $09 / 21 / 94$ & -- & $\mathrm{D}$ & -- \\
\hline 90 & 171 & S01 E59 27CA1 & 374947115203801 & 374947 & 1152038 & 200 & 5,020 & $01 / 01 / 80$ & -- & $\mathrm{D}$ & -- \\
\hline \multirow[t]{13}{*}{91} & 172 & N02 E58 03AA1 & 380348115265001 & 380420 & 1152618 & 200 & 5,200 & $10 / 01 / 80$ & 145 & -- & $\mathbf{R}$ \\
\hline & & & & & & & & $01 / 01 / 81$ & 139 & -- & $\mathbf{R}$ \\
\hline & & & & & & & & $03 / 01 / 81$ & 140 & -- & $\mathrm{R}$ \\
\hline & & & & & & & & $03 / 12 / 85$ & 138.27 & - & $\mathrm{S}$ \\
\hline & & & & & & & & $03 / 20 / 90$ & 136.7 & -- & $\mathrm{T}$ \\
\hline & & & & & & & & $09 / 04 / 91$ & -- & $\mathrm{O}$ & -- \\
\hline & & & & & & & & $03 / 26 / 92$ & -- & $\mathrm{O}$ & -- \\
\hline & & & & & & & & $06 / 18 / 92$ & -- & $\mathrm{O}$ & -- \\
\hline & & & & & & & & $09 / 10 / 92$ & -- & $\mathrm{O}$ & -- \\
\hline & & & & & & & & $12 / 23 / 92$ & - & $\mathrm{O}$ & -- \\
\hline & & & & & & & & $04 / 01 / 93$ & -- & $\mathrm{O}$ & -- \\
\hline & & & & & & & & $06 / 08 / 93$ & -- & $\mathrm{O}$ & -- \\
\hline & & & & & & & & $03 / 23 / 94$ & -- & $\mathrm{O}$ & -- \\
\hline \multirow[t]{10}{*}{92} & 172 & N03 E58 01DA1 & 380835115242601 & 380911 & 1152404 & 100 & 5,200 & $10 / 01 / 80$ & 85 & -- & $\mathrm{R}$ \\
\hline & & & & & & & & $01 / 01 / 81$ & 87 & -- & $\mathbf{R}$ \\
\hline & & & & & & & & $03 / 01 / 81$ & 88 & -- & $\mathbf{R}$ \\
\hline & & & & & & & & $03 / 12 / 85$ & 86.69 & -- & $\mathrm{S}$ \\
\hline & & & & & & & & $03 / 20 / 90$ & 84.6 & -- & $\mathbf{T}$ \\
\hline & & & & & & & & $09 / 04 / 91$ & 85.1 & -- & $\mathrm{T}$ \\
\hline & & & & & & & & $03 / 26 / 92$ & 84.74 & -- & $\mathrm{S}$ \\
\hline & & & & & & & & $06 / 18 / 92$ & 84.65 & -- & $\mathrm{S}$ \\
\hline & & & & & & & & $09 / 10 / 92$ & 84.56 & -- & $\mathrm{S}$ \\
\hline & & & & & & & & $10 / 07 / 92$ & 84.55 & -- & $\mathrm{S}$ \\
\hline
\end{tabular}


Table 1. Ground-water data for small-diameter monitoring wells - Continued

\begin{tabular}{|c|c|c|c|c|c|c|c|c|c|c|c|}
\hline \multirow[b]{2}{*}{$\begin{array}{c}\text { Map } \\
\text { no. } \\
\text { (pl. 1) }\end{array}$} & \multicolumn{3}{|c|}{ U.S. Geological Survey site designations } & \multirow[b]{2}{*}{ Latitude } & \multirow[b]{2}{*}{ Longitude } & \multirow[b]{2}{*}{$\begin{array}{l}\text { Well } \\
\text { depth } \\
\text { (feet) }\end{array}$} & \multirow{2}{*}{$\begin{array}{l}\text { Land- } \\
\text { surface } \\
\text { altitude } \\
\text { (feet above } \\
\text { sea level) }\end{array}$} & \multicolumn{4}{|c|}{ Water level } \\
\hline & & Local well number & $\begin{array}{c}\text { Standard } \\
\text { identification }\end{array}$ & & & & & Date $^{1}$ & $\begin{array}{c}\text { Feet } \\
\text { below } \\
\text { land } \\
\text { surface }^{2}\end{array}$ & $\begin{array}{c}\text { Site } \\
\text { status }\end{array}$ & Method \\
\hline \multirow[t]{10}{*}{92} & 172 & N03 E58 01DA1 & 380835115242601 & 380911 & 1152404 & 100 & 5,200 & $12 / 23 / 92$ & 84.62 & -- & $\mathrm{S}$ \\
\hline & & & & & & & & $04 / 01 / 93$ & 84.39 & -- & $\mathbf{S}$ \\
\hline & & & & & & & & $06 / 08 / 93$ & 84.40 & -- & $\mathbf{S}$ \\
\hline & & & & & & & & $09 / 08 / 93$ & 84.30 & -- & $\mathrm{S}$ \\
\hline & & & & & & & & $12 / 16 / 93$ & 84.23 & -- & $\mathrm{S}$ \\
\hline & & & & & & & & $03 / 14 / 94$ & 37.3 & -- & $\mathrm{T}$ \\
\hline & & & & & & & & $03 / 23 / 94$ & 84.07 & -- & $\mathrm{S}$ \\
\hline & & & & & & & & $03 / 28 / 94$ & 84.10 & -- & $\mathrm{S}$ \\
\hline & & & & & & & & $07 / 06 / 94$ & 84.14 & -- & $\mathrm{S}$ \\
\hline & & & & & & & & $09 / 21 / 94$ & 84.03 & -- & $\mathrm{S}$ \\
\hline \multirow[t]{6}{*}{93} & 172 & N03 E59 18BB1 & 380646115235001 & 380733 & 1152355 & 200 & 5,200 & $10 / 01 / 80$ & 154 & -- & $\mathrm{R}$ \\
\hline & & & & & & & & $01 / 01 / 81$ & 152 & -- & $\mathrm{R}$ \\
\hline & & & & & & & & $03 / 01 / 81$ & 153 & -- & $\mathrm{R}$ \\
\hline & & & & & & & & $03 / 12 / 85$ & 151.47 & -- & $\mathrm{S}$ \\
\hline & & & & & & & & $03 / 20 / 85$ & 149.9 & -- & $\mathrm{T}$ \\
\hline & & & & & & & & $09 / 04 / 91$ & 150.14 & -- & $\mathrm{S}$ \\
\hline 94 & 172 & N04 E58 22DB1 & 381127115263501 & 381127 & 1152635 & 100 & 5,520 & $01 / 01 / 81$ & -- & $\mathrm{D}$ & -- \\
\hline \multirow[t]{5}{*}{95} & 172 & N04 E58 22DB2 & 381127115263502 & 381127 & 1152635 & 398 & 5,520 & $09 / 14 / 93$ & 184.7 & -- & $\mathrm{T}$ \\
\hline & & & & & & & & $01 / 20 / 94$ & 184.2 & -- & $\mathrm{T}$ \\
\hline & & & & & & & & $03 / 23 / 94$ & 184.0 & -- & $\mathrm{T}$ \\
\hline & & & & & & & & $07 / 06 / 94$ & 183.80 & -- & $\mathrm{S}$ \\
\hline & & & & & & & & $09 / 21 / 94$ & 183.75 & -- & $\mathrm{S}$ \\
\hline 96 & 172 & N04 E58 33DB1 & 380942115274201 & 380942 & 1152742 & 200 & 5,600 & $11 / 01 / 80$ & -- & $\mathrm{D}$ & -- \\
\hline \multirow[t]{5}{*}{97} & 172 & N04 E58 33DB2 & 380942115274202 & 380942 & 1152742 & 400 & 5,520 & $09 / 14 / 93$ & 392.7 & -- & $\mathrm{T}$ \\
\hline & & & & & & & & $01 / 20 / 94$ & 392.7 & -- & $\mathbf{T}$ \\
\hline & & & & & & & & $03 / 23 / 94$ & 392.4 & -- & $\mathrm{T}$ \\
\hline & & & & & & & & $07 / 06 / 94$ & 394.1 & -- & $\mathrm{T}$ \\
\hline & & & & & & & & $09 / 21 / 94$ & 393.23 & -- & $\mathrm{S}$ \\
\hline \multirow[t]{3}{*}{98} & 172 & N04 E59 30CD1 & 380937115233401 & 381039 & 1152334 & 100 & 5,240 & $01 / 01 / 81$ & 64 & -- & $\mathrm{R}$ \\
\hline & & & & & & & & $03 / 01 / 81$ & 65 & -- & $\mathrm{R}$ \\
\hline & & & & & & & & $03 / 12 / 85$ & 62.98 & -- & $\mathrm{S}$ \\
\hline \multirow[t]{16}{*}{99} & 172 & N05 E59 31CAA1 & 381457115232901 & 381516 & 1152332 & 200 & 5,479 & $10 / 01 / 80$ & 115 & -- & $\mathrm{R}$ \\
\hline & & & & & & & & $11 / 01 / 80$ & 111 & -- & $\mathrm{R}$ \\
\hline & & & & & & & & $01 / 01 / 81$ & 113 & -- & $\mathrm{R}$ \\
\hline & & & & & & & & $03 / 20 / 90$ & 110.7 & -- & $\mathrm{T}$ \\
\hline & & & & & & & & $09 / 04 / 91$ & 113.0 & -- & $\mathrm{T}$ \\
\hline & & & & & & & & $03 / 26 / 92$ & 111.10 & -- & $\mathrm{S}$ \\
\hline & & & & & & & & $06 / 18 / 92$ & 111.43 & -- & $\mathrm{S}$ \\
\hline & & & & & & & & $09 / 10 / 92$ & 111.31 & -- & $\mathrm{S}$ \\
\hline & & & & & & & & $12 / 23 / 92$ & 111.42 & -- & $\mathrm{S}$ \\
\hline & & & & & & & & $04 / 01 / 93$ & 111.80 & -- & $\mathrm{S}$ \\
\hline & & & & & & & & $06 / 08 / 93$ & 111.75 & -- & $\mathrm{S}$ \\
\hline & & & & & & & & $09 / 08 / 93$ & 111.91 & -- & $\mathrm{S}$ \\
\hline & & & & & & & & $12 / 16 / 93$ & 111.35 & -- & $\mathrm{S}$ \\
\hline & & & & & & & & $03 / 28 / 94$ & 111.44 & -- & $\mathrm{S}$ \\
\hline & & & & & & & & $07 / 06 / 94$ & 112.34 & -- & $\mathrm{S}$ \\
\hline & & & & & & & & $09 / 21 / 94$ & 111.20 & -- & $\mathrm{S}$ \\
\hline
\end{tabular}


Table 1. Ground-water data for small-diameter monitoring wells - Continued

\begin{tabular}{|c|c|c|c|c|c|c|c|c|c|c|}
\hline \multirow[b]{2}{*}{$\begin{array}{c}\text { Map } \\
\text { no. } \\
\text { (pl. 1) }\end{array}$} & \multicolumn{2}{|c|}{ U.S. Geological Survey site designations } & \multirow[b]{2}{*}{ Latitude } & \multirow[b]{2}{*}{ Longitude } & \multirow[b]{2}{*}{$\begin{array}{c}\text { Well } \\
\text { depth } \\
\text { (feet) }\end{array}$} & \multirow{2}{*}{$\begin{array}{l}\text { Land- } \\
\text { surface } \\
\text { altitude } \\
\text { (feet above } \\
\text { sea level) }\end{array}$} & \multicolumn{4}{|c|}{ Water level } \\
\hline & Local well number & $\begin{array}{c}\text { Standard } \\
\text { identification }\end{array}$ & & & & & Date $^{1}$ & $\begin{array}{c}\text { Feet } \\
\text { below } \\
\text { land } \\
\text { surface }^{2}\end{array}$ & $\begin{array}{l}\text { Site } \\
\text { status }\end{array}$ & Method \\
\hline \multirow[t]{4}{*}{100} & 173A N02 E52 24DC1 & 375619116035301 & 380103 & 1160414 & 200 & 4,880 & $09 / 01 / 80$ & 122 & -- & $\mathrm{R}$ \\
\hline & & & & & & & $01 / 01 / 81$ & 119 & -- & $\mathrm{R}$ \\
\hline & & & & & & & $03 / 22 / 93$ & 77.0 & -- & $\mathrm{T}$ \\
\hline & & & & & & & $03 / 26 / 93$ & 76.90 & -- & $\mathrm{S}$ \\
\hline 101 & 173A N02 E52 35AC1 & 375850116051801 & 375850 & 1160518 & 200 & 4,970 & $03 / 01 / 81$ & -- & $\mathrm{D}$ & -- \\
\hline \multirow[t]{3}{*}{102} & 173A N02 E53 09BC1 & 380313116012601 & 380313 & 1160126 & 200 & 4,856 & $09 / 01 / 80$ & 167 & -- & $\mathrm{R}$ \\
\hline & & & & & & & $05 / 15 / 84$ & 158.20 & -- & $\mathrm{S}$ \\
\hline & & & & & & & $09 / 04 / 91$ & 158.01 & -- & $\mathrm{S}$ \\
\hline \multirow[t]{3}{*}{103} & 173A N02 E53 27DA1 & 380018115592901 & 380018 & 1155929 & 150 & 4,865 & $09 / 01 / 80$ & 88 & -- & $\mathrm{R}$ \\
\hline & & & & & & & $05 / 15 / 84$ & 84.56 & -- & $\mathrm{S}$ \\
\hline & & & & & & & $03 / 15 / 90$ & 87.33 & -- & $\mathrm{S}$ \\
\hline \multirow[t]{2}{*}{104} & 173A N02 E53 35AA1 & 375951115582201 & 375951 & 1155822 & 200 & 4,990 & $09 / 01 / 80$ & 187 & -- & $\mathrm{R}$ \\
\hline & & & & & & & $01 / 01 / 81$ & 192 & -- & $\mathrm{R}$ \\
\hline \multirow[t]{2}{*}{105} & 173A N03 E53 18BCl & 380740116034101 & 380740 & 1160341 & 200 & 4,990 & $03 / 01 / 81$ & -- & $\mathrm{D}$ & -- \\
\hline & & & & & & & $03 / 15 / 90$ & 17.0 & - & $\mathrm{T}$ \\
\hline \multirow[t]{2}{*}{106} & 173B N04 E54 24ABDC1 & 381146115442401 & 381209 & 1155051 & 200 & 4,950 & $09 / 01 / 80$ & 177 & - & $\mathrm{R}$ \\
\hline & & & & & & & $03 / 01 / 81$ & 166 & - & $\mathrm{R}$ \\
\hline \multirow[t]{10}{*}{107} & 173B N05 E54 26DCBA1 & 381603115520901 & 381550 & 1155202 & 200 & 4,835 & $09 / 01 / 80$ & 72 & - & $\mathrm{R}$ \\
\hline & & & & & & & $01 / 01 / 81$ & 71 & -- & $\mathrm{R}$ \\
\hline & & & & & & & $05 / 15 / 84$ & 70.35 & -- & $\mathrm{S}$ \\
\hline & & & & & & & $03 / 08 / 90$ & 69.40 & -- & $\mathrm{S}$ \\
\hline & & & & & & & $09 / 04 / 91$ & 69.33 & -- & $\mathrm{S}$ \\
\hline & & & & & & & $04 / 06 / 93$ & 69.76 & -- & $\mathrm{S}$ \\
\hline & & & & & & & $09 / 17 / 93$ & 69.92 & -- & $\mathrm{S}$ \\
\hline & & & & & & & $01 / 05 / 94$ & 69.86 & -- & $\mathrm{S}$ \\
\hline & & & & & & & $03 / 28 / 94$ & 69.78 & -- & $\mathrm{S}$ \\
\hline & & & & & & & $09 / 21 / 94$ & 69.94 & - & $\mathrm{S}$ \\
\hline \multirow[t]{5}{*}{108} & 173B N05 E56 21ABCC2 & 381651115405901 & 381651 & 1154059 & 200 & 4,940 & $09 / 01 / 80$ & 153 & -- & $\mathrm{R}$ \\
\hline & & & & & & & $01 / 01 / 81$ & 194 & -- & $\mathrm{R}$ \\
\hline & & & & & & & $04 / 07 / 93$ & 196.81 & -- & $\mathrm{S}$ \\
\hline & & & & & & & $09 / 14 / 93$ & 196.94 & -- & $\mathrm{S}$ \\
\hline & & & & & & & $12 / 22 / 93$ & 196.87 & -- & $\mathrm{S}$ \\
\hline \multirow[t]{15}{*}{109} & 173B N06 E54 23BD1 & 382144115542601 & 382148 & 1155212 & 200 & 4,790 & $01 / 01 / 81$ & 30 & -- & $\mathrm{R}$ \\
\hline & & & & & & & $03 / 01 / 81$ & 29 & -- & $\mathrm{R}$ \\
\hline & & & & & & & $05 / 15 / 84$ & 29.32 & -- & $\mathrm{S}$ \\
\hline & & & & & & & $03 / 08 / 90$ & 29.06 & -- & $\mathrm{S}$ \\
\hline & & & & & & & $09 / 04 / 91$ & 29.21 & - & $\mathrm{S}$ \\
\hline & & & & & & & $03 / 26 / 92$ & 29.17 & -- & $\mathrm{S}$ \\
\hline & & & & & & & $06 / 18 / 92$ & 29.19 & -- & $\mathrm{S}$ \\
\hline & & & & & & & $08 / 18 / 92$ & 29.22 & -- & $\mathrm{S}$ \\
\hline & & & & & & & $09 / 17 / 92$ & 29.23 & -- & $\mathrm{S}$ \\
\hline & & & & & & & $12 / 10 / 92$ & 29.10 & -- & $\mathrm{S}$ \\
\hline & & & & & & & $03 / 23 / 93$ & 29.7 & -- & $\mathrm{T}$ \\
\hline & & & & & & & $06 / 17 / 93$ & -- & W & -- \\
\hline & & & & & & & $01 / 05 / 94$ & 29.30 & - & $\mathrm{S}$ \\
\hline & & & & & & & $03 / 28 / 94$ & 29.27 & -- & $\mathrm{S}$ \\
\hline & & & & & & & $09 / 21 / 94$ & 29.17 & - & $\mathrm{S}$ \\
\hline
\end{tabular}


Table 1. Ground-water data for small-diameter monitoring wells -Continued

\begin{tabular}{|c|c|c|c|c|c|c|c|c|c|c|}
\hline \multirow[b]{2}{*}{$\begin{array}{c}\text { Map } \\
\text { no. } \\
\text { (pl. 1) }\end{array}$} & \multicolumn{2}{|c|}{ U.S. Geological Survey site designations } & \multirow[b]{2}{*}{ Latitude } & \multirow[b]{2}{*}{ Longitude } & \multirow[b]{2}{*}{$\begin{array}{l}\text { Well } \\
\text { depth } \\
\text { (feet) }\end{array}$} & \multirow{2}{*}{$\begin{array}{c}\text { Land- } \\
\text { surface } \\
\text { altitude } \\
\text { (feet above } \\
\text { sea level) }\end{array}$} & \multicolumn{4}{|c|}{ Water level } \\
\hline & Local well number & $\begin{array}{c}\text { Standard } \\
\text { identification }\end{array}$ & & & & & Date $^{1}$ & $\begin{array}{c}\text { Feet } \\
\text { below } \\
\text { land } \\
\text { surface }^{2}\end{array}$ & $\begin{array}{l}\text { Site } \\
\text { status }\end{array}$ & Method \\
\hline \multirow[t]{2}{*}{110} & 173B N06 E56 36CA1 & 382009115374201 & 382009 & 1153742 & 150 & 5,100 & $09 / 01 / 80$ & 148 & -- & $\mathrm{R}$ \\
\hline & & & & & & & $01 / 01 / 81$ & -- & $\mathrm{D}$ & -- \\
\hline 111 & 173B N07 E55 08AC1 & 382844115484301 & 382844 & 1154843 & -- & 4,863 & $03 / 01 / 81$ & 93 & & $\mathrm{R}$ \\
\hline \multirow[t]{4}{*}{112} & 173B N07 E57 11BCDD1 & 382855115321801 & 382855 & 1153218 & 350 & 4,840 & $01 / 01 / 81$ & -- & $\mathrm{D}$ & -- \\
\hline & & & & & & & $03 / 01 / 81$ & -- & D & -- \\
\hline & & & & & & & $09 / 16 / 93$ & 120.4 & -- & $\mathrm{T}$ \\
\hline & & & & & & & $12 / 22 / 93$ & 124.85 & -- & $\mathrm{S}$ \\
\hline 113 & 173B N07 E57 11DB1 & 382850115315401 & 382850 & 1153154 & 118 & 4,940 & $03 / 01 / 81$ & -- & $\mathrm{D}$ & -- \\
\hline \multirow[t]{17}{*}{114} & 173B N09 E56 20CD1 & 383712115420301 & 383712 & 1154203 & 198 & 4,905 & $01 / 01 / 81$ & 110 & -- & $\mathrm{R}$ \\
\hline & & & & & & & $03 / 01 / 81$ & 110 & -- & $\mathrm{R}$ \\
\hline & & & & & & & $06 / 05 / 84$ & 109.71 & -- & $\mathrm{S}$ \\
\hline & & & & & & & $03 / 02 / 90$ & 109.68 & -- & $\mathrm{S}$ \\
\hline & & & & & & & $09 / 03 / 91$ & 109.54 & -- & $\mathrm{S}$ \\
\hline & & & & & & & $03 / 26 / 92$ & 108.57 & -- & $\mathrm{S}$ \\
\hline & & & & & & & $06 / 24 / 92$ & 109.48 & -- & $\mathrm{S}$ \\
\hline & & & & & & & $08 / 18 / 92$ & 109.54 & -- & $\mathrm{S}$ \\
\hline & & & & & & & $09 / 17 / 92$ & 110.28 & -- & $\mathrm{S}$ \\
\hline & & & & & & & $12 / 09 / 92$ & 110.38 & -- & $\mathrm{S}$ \\
\hline & & & & & & & $04 / 08 / 93$ & 110.35 & - & $S$ \\
\hline & & & & & & & $07 / 01 / 93$ & 110.20 & -- & $\mathrm{S}$ \\
\hline & & & & & & & $09 / 17 / 93$ & 110.32 & -- & $\mathrm{S}$ \\
\hline & & & & & & & $12 / 21 / 93$ & 110.33 & - & $\mathrm{S}$ \\
\hline & & & & & & & $03 / 28 / 94$ & 110.08 & -- & $\mathrm{S}$ \\
\hline & & & & & & & $07 / 06 / 94$ & 110.21 & -- & $S$ \\
\hline & & & & & & & $09 / 20 / 94$ & 110.30 & -- & $\mathrm{S}$ \\
\hline 115 & 173B N10 E56 03AA1 & 384537115391201 & 384537 & 1153912 & 200 & 5,180 & $01 / 01 / 81$ & -- & $\mathrm{D}$ & -- \\
\hline \multirow[t]{5}{*}{116} & 173B N10 E56 34CC1 & 384042115400301 & 384043 & 1154008 & 199 & 4,987 & $01 / 01 / 81$ & 153 & -- & $\mathbf{R}$ \\
\hline & & & & & & & $03 / 01 / 81$ & 153 & -- & $\mathrm{R}$ \\
\hline & & & & & & & $06 / 05 / 84$ & 151.93 & -- & $\mathrm{S}$ \\
\hline & & & & & & & $03 / 06 / 90$ & 150.5 & -- & $\mathrm{T}$ \\
\hline & & & & & & & 09/03/91 & 149.75 & -- & $\mathrm{S}$ \\
\hline 117 & 173B N11 E57 26BD1 & 384705115314501 & 384705 & 1153145 & 199 & 5,060 & $01 / 01 / 81$ & -- & $\mathrm{D}$ & -- \\
\hline 118 & 173B N13 E55H18BAl & 385811115494801 & 385811 & 1154404 & 200 & 5,630 & $01 / 01 / 81$ & 123 & -- & $\mathrm{R}$ \\
\hline \multirow[t]{4}{*}{119} & 173B N15 E56 25BB1 & 390829115373801 & 390831 & 1153740 & 158 & 5,950 & $01 / 01 / 80$ & 128 & -- & $\mathrm{R}$ \\
\hline & & & & & & & $01 / 01 / 81$ & 128 & -- & $\mathrm{R}$ \\
\hline & & & & & & & $03 / 01 / 81$ & 127 & -- & $\mathrm{R}$ \\
\hline & & & & & & & $09 / 03 / 91$ & 125.09 & -- & $\mathrm{S}$ \\
\hline 120 & 174 N16 E59 12DB1 & 391556115163901 & 391556 & 1151639 & 101 & 6,300 & $01 / 01 / 81$ & -- & $\mathrm{D}$ & -- \\
\hline 121 & 174 N16 E60 33CD1 & 391209115133301 & 391209 & 1151333 & 161 & 6,171 & $01 / 01 / 81$ & -- & $\mathrm{D}$ & -- \\
\hline 122 & 175 N21 E58 17CA1 & 394117115275801 & 394117 & 1152758 & 200 & 6,151 & $01 / 01 / 81$ & 103 & -- & $\mathrm{R}$ \\
\hline \multirow[t]{2}{*}{123} & 175 N21 E58 21DB1 & 394024115263401 & 394024 & 1152634 & 150 & 6,234 & $01 / 01 / 81$ & 55 & -- & $\mathrm{R}$ \\
\hline & & & & & & & $10 / 01 / 91$ & 54.26 & -- & $\mathrm{S}$ \\
\hline 124 & 175 N21 E58 25BA1 & 393956115232701 & 393956 & 1152327 & 150 & 6,105 & $01 / 01 / 81$ & 77 & -- & $\mathrm{R}$ \\
\hline \multirow[t]{3}{*}{125} & 175 N22 E58 34DC1 & 394340115252501 & 394351 & 1152522 & 150 & 6,234 & $01 / 01 / 81$ & 49 & -- & $\mathrm{R}$ \\
\hline & & & & & & & $03 / 01 / 81$ & 50 & -- & $\mathrm{R}$ \\
\hline & & & & & & & $09 / 30 / 91$ & 48.14 & -- & $\mathrm{S}$ \\
\hline
\end{tabular}


Table 1. Ground-water data for small-diameter monitoring wells - Continued

\begin{tabular}{|c|c|c|c|c|c|c|c|c|c|c|}
\hline \multirow[b]{2}{*}{$\begin{array}{c}\text { Map } \\
\text { no. } \\
\text { (pl. 1) }\end{array}$} & \multicolumn{2}{|c|}{ U.S. Geological Survey site designations } & \multirow[b]{2}{*}{ Latitude } & \multirow[b]{2}{*}{ Longitude } & \multirow[b]{2}{*}{$\begin{array}{c}\text { Well } \\
\text { depth } \\
\text { (feet) }\end{array}$} & \multirow{2}{*}{$\begin{array}{c}\text { Land- } \\
\text { surface } \\
\text { altitude } \\
\text { (feet above } \\
\text { sea level) }\end{array}$} & \multicolumn{4}{|c|}{ Water level } \\
\hline & Local well number & $\begin{array}{c}\text { Standard } \\
\text { identification }\end{array}$ & & & & & Date $^{1}$ & $\begin{array}{c}\text { Feet } \\
\text { below } \\
\text { land } \\
\text { surface }^{2}\end{array}$ & $\begin{array}{l}\text { Site } \\
\text { status }\end{array}$ & Method \\
\hline 126 & 175 N22 E59 15DCl & 394617115183401 & 394617 & 1151834 & 200 & 6,234 & $\begin{array}{l}01 / 01 / 81 \\
10 / 02 / 91\end{array}$ & $\begin{array}{l}45 \\
44.66\end{array}$ & -- & $\begin{array}{l}\mathrm{R} \\
\mathrm{S}\end{array}$ \\
\hline 127 & 175 N22 E59 32CCCB1 & 394343115213301 & 394343 & 1152133 & 203 & 6,097 & $\begin{array}{l}01 / 01 / 81 \\
04 / 21 / 83 \\
04 / 24 / 84\end{array}$ & $\begin{array}{l}70 \\
70.25 \\
70.25\end{array}$ & $\begin{array}{l}-- \\
-- \\
--\end{array}$ & $\begin{array}{l}\mathrm{R} \\
\mathrm{S} \\
\mathrm{S}\end{array}$ \\
\hline 128 & 175 N23 E58 27CA1 & 395013115252801 & 395012 & 1152538 & 150 & 6,234 & $\begin{array}{l}01 / 01 / 81 \\
03 / 01 / 81 \\
04 / 20 / 83 \\
04 / 24 / 84 \\
10 / 01 / 91\end{array}$ & $\begin{array}{l}107 \\
107 \\
106.04 \\
104.52 \\
105.10\end{array}$ & $\begin{array}{l}-- \\
-- \\
-- \\
-- \\
--\end{array}$ & $\begin{array}{l}\mathrm{R} \\
\mathrm{R} \\
\mathrm{S} \\
\mathrm{S} \\
\mathrm{S}\end{array}$ \\
\hline 129 & 175 N23 E59 06CD1 & 395327115213101 & 395323 & 1152221 & 150 & 6,234 & $\begin{array}{l}01 / 01 / 81 \\
03 / 01 / 81 \\
04 / 20 / 83 \\
10 / 01 / 91\end{array}$ & $\begin{array}{l}73 \\
72 \\
72.59 \\
71.70\end{array}$ & $\begin{array}{l}-- \\
-- \\
-- \\
--\end{array}$ & $\begin{array}{l}\mathrm{R} \\
\mathrm{R} \\
\mathrm{S} \\
\mathrm{S}\end{array}$ \\
\hline 130 & 175 N23 E59 16CD1 & 395154115201701 & 395133 & 1151956 & 150 & 6,234 & $\begin{array}{l}01 / 01 / 81 \\
03 / 01 / 81 \\
04 / 21 / 83 \\
10 / 02 / 91\end{array}$ & $\begin{array}{l}62 \\
62 \\
61.60 \\
61.08\end{array}$ & $\begin{array}{l}-- \\
-- \\
-- \\
--\end{array}$ & $\begin{array}{l}\mathrm{R} \\
\mathrm{R} \\
\mathrm{S} \\
\mathrm{S}\end{array}$ \\
\hline 131 & 175 N24 E59 01DC1 & 395845115161901 & 395846 & 1151613 & 150 & 6,235 & $\begin{array}{l}01 / 01 / 81 \\
03 / 01 / 81 \\
04 / 20 / 83 \\
10 / 01 / 91\end{array}$ & $\begin{array}{l}104 \\
103 \\
103.25 \\
102.50\end{array}$ & $\begin{array}{l}-- \\
-- \\
-- \\
--\end{array}$ & $\begin{array}{l}\mathrm{R} \\
\mathrm{R} \\
\mathrm{S} \\
\mathrm{S}\end{array}$ \\
\hline 132 & 175 N24 E59 27AB1 & 395535115184601 & 395549 & 1151847 & 151 & 6,234 & $\begin{array}{l}01 / 01 / 81 \\
04 / 20 / 83 \\
10 / 01 / 91\end{array}$ & $\begin{array}{l}57 \\
58.01 \\
55.47\end{array}$ & $\begin{array}{l}-- \\
-- \\
-\end{array}$ & $\begin{array}{l}\mathrm{R} \\
\mathrm{S} \\
\mathrm{S}\end{array}$ \\
\hline $\begin{array}{l}133 \\
134\end{array}$ & $\begin{array}{l}175 \text { N25 E60 17AA1 } \\
178 \mathrm{~B} \text { N20 E62 32BCAA1 }\end{array}$ & $\begin{array}{l}400303115134501 \\
393338115013001\end{array}$ & $\begin{array}{l}400303 \\
393338\end{array}$ & $\begin{array}{l}1151345 \\
1150130\end{array}$ & $\begin{array}{l}201 \\
200\end{array}$ & $\begin{array}{l}6,800 \\
6,350\end{array}$ & $\begin{array}{l}01 / 01 / 81 \\
01 / 01 / 81 \\
03 / 01 / 81 \\
04 / 20 / 83\end{array}$ & $\begin{array}{l}-- \\
141 \\
142 \\
141.29\end{array}$ & $\begin{array}{l}\text { D } \\
-- \\
-- \\
--\end{array}$ & $\begin{array}{l}-- \\
\mathrm{R} \\
\mathrm{R} \\
\mathrm{S}\end{array}$ \\
\hline 135 & 178B N21 E60 12AB1 & 394232115092701 & 394242 & 1150928 & 197 & 6,398 & $\begin{array}{l}01 / 01 / 81 \\
10 / 09 / 91\end{array}$ & $\begin{array}{l}89 \\
88.86\end{array}$ & $\begin{array}{l}-- \\
--\end{array}$ & $\begin{array}{l}\mathrm{R} \\
\mathrm{S}\end{array}$ \\
\hline 136 & 178B N21 E61 07AC1 & 394232115081101 & 394226 & 1150713 & 139 & 6,398 & $\begin{array}{l}01 / 01 / 81 \\
10 / 09 / 91\end{array}$ & $\begin{array}{l}80 \\
79.19\end{array}$ & -- & $\begin{array}{l}\mathrm{R} \\
\mathrm{S}\end{array}$ \\
\hline 137 & 178B N21 E61 22AA1 & 394020115043001 & 394048 & 1150331 & 175 & 6,398 & $\begin{array}{l}01 / 01 / 81 \\
04 / 20 / 83 \\
10 / 10 / 91\end{array}$ & $\begin{array}{l}57 \\
56.77 \\
54.08\end{array}$ & $\begin{array}{l}-- \\
-- \\
--\end{array}$ & $\begin{array}{l}\mathrm{R} \\
\mathrm{S} \\
\mathrm{S}\end{array}$ \\
\hline 138 & 178B N21 E61 30BD1 & 393926115085201 & 393926 & 1150832 & 186 & 6,398 & $\begin{array}{l}01 / 01 / 81 \\
10 / 10 / 91\end{array}$ & $\begin{array}{l}137 \\
136.12\end{array}$ & -- & $\begin{array}{l}\mathrm{R} \\
\mathrm{S}\end{array}$ \\
\hline 139 & 178B N21 E61 33DC1 & 393807115080301 & 393825 & 1150645 & 193 & 6,275 & $\begin{array}{l}01 / 01 / 81 \\
04 / 20 / 83 \\
10 / 09 / 91\end{array}$ & $\begin{array}{l}77 \\
77.84 \\
76.93\end{array}$ & $\begin{array}{l}-- \\
-- \\
--\end{array}$ & $\begin{array}{l}\mathrm{R} \\
\mathrm{S} \\
\mathrm{S}\end{array}$ \\
\hline 140 & 178B N22 E60 09DB1 & 394709115124501 & 394746 & 1151251 & 160 & 6,234 & $\begin{array}{l}01 / 01 / 81 \\
04 / 20 / 83 \\
10 / 02 / 91\end{array}$ & $\begin{array}{l}48 \\
50.18 \\
47.82\end{array}$ & -- & $\begin{array}{l}\mathrm{R} \\
\mathrm{S} \\
\mathrm{S}\end{array}$ \\
\hline 141 & 178B N23 E61 08DDCA1 & 395211115054301 & 395211 & 1150543 & 145 & 6,338 & $\begin{array}{l}01 / 01 / 81 \\
04 / 18 / 83\end{array}$ & $\begin{array}{l}104 \\
105.08\end{array}$ & $\begin{array}{l}-- \\
--\end{array}$ & $\begin{array}{l}\mathrm{R} \\
\mathrm{S}\end{array}$ \\
\hline
\end{tabular}


Table 1. Ground-water data for small-diameter monitoring wells - Continued

\begin{tabular}{|c|c|c|c|c|c|c|c|c|c|c|}
\hline \multirow[b]{2}{*}{$\begin{array}{c}\text { Map } \\
\text { no. } \\
\text { (pl. 1) }\end{array}$} & \multicolumn{2}{|c|}{ U.S. Geological Survey site designations } & \multirow[b]{2}{*}{ Latitude } & \multirow[b]{2}{*}{ Longitude } & \multirow[b]{2}{*}{$\begin{array}{r}\text { Well } \\
\text { depth } \\
\text { (feet) }\end{array}$} & \multirow{2}{*}{$\begin{array}{l}\text { Land- } \\
\text { surface } \\
\text { altitude } \\
\text { (feet above } \\
\text { sea level) }\end{array}$} & \multicolumn{4}{|c|}{ Water level } \\
\hline & Local well number & $\begin{array}{c}\text { Standard } \\
\text { identification }\end{array}$ & & & & & Date $^{1}$ & $\begin{array}{c}\text { Feet } \\
\text { below } \\
\text { land } \\
\text { surface }^{2}\end{array}$ & $\begin{array}{l}\text { Site } \\
\text { status }\end{array}$ & Method \\
\hline 142 & 178B N24 E61 04DD1 & 395827115050801 & 395831 & 1150425 & 201 & 6,398 & $\begin{array}{l}01 / 01 / 81 \\
10 / 08 / 91\end{array}$ & $\begin{array}{c}14 \\
--\end{array}$ & $\overline{\mathrm{O}}$ & $\begin{array}{l}\mathrm{R} \\
--\end{array}$ \\
\hline 143 & 178B N24 E61 06DA1 & 395853115075101 & 395848 & 1150636 & 177 & 6,398 & $\begin{array}{l}01 / 01 / 81 \\
04 / 18 / 83 \\
10 / 08 / 91\end{array}$ & $\begin{array}{l}77 \\
77.82 \\
77.57\end{array}$ & $\begin{array}{l}-- \\
-- \\
--\end{array}$ & $\begin{array}{l}\mathrm{R} \\
\mathrm{S} \\
\mathrm{S}\end{array}$ \\
\hline 144 & 178B N26 E61 28CA1 & 400557115055701 & 400601 & 1150610 & 148 & 6,398 & $\begin{array}{l}01 / 01 / 81 \\
04 / 18 / 83 \\
10 / 12 / 91\end{array}$ & $\begin{array}{l}138 \\
136.64 \\
136.01\end{array}$ & $\begin{array}{l}-- \\
-- \\
--\end{array}$ & $\begin{array}{l}\mathrm{R} \\
\mathrm{S} \\
\mathrm{S}\end{array}$ \\
\hline 145 & 178B N26 E62 19BBD1 & 400708115015501 & 400708 & 1150155 & 201 & 6,290 & $\begin{array}{l}01 / 01 / 81 \\
04 / 18 / 83\end{array}$ & $\begin{array}{l}33 \\
33.86\end{array}$ & -- & $\begin{array}{l}\mathrm{R} \\
\mathrm{S}\end{array}$ \\
\hline 146 & $179 \mathrm{~N} 13 \mathrm{E} 6401 \mathrm{CCl}$ & 390048114442901 & 390048 & 1144429 & 200 & 6,880 & $\begin{array}{l}07 / 29 / 80 \\
03 / 01 / 81\end{array}$ & -- & $\begin{array}{l}\mathrm{D} \\
\mathrm{D}\end{array}$ & - \\
\hline 147 & 179 N13 E64 06BA1 & 390128114495401 & 390125 & 1144939 & 200 & 6,815 & $\begin{array}{l}11 / 01 / 80 \\
01 / 01 / 81 \\
03 / 01 / 81 \\
03 / 03 / 81 \\
05 / 09 / 83 \\
08 / 21 / 84 \\
04 / 21 / 85 \\
09 / 27 / 91 \\
03 / 15 / 94\end{array}$ & $\begin{array}{l}18 \\
26 \\
27 \\
26.39 \\
32.4 \\
\\
34.44 \\
35.57 \\
44.55 \\
37.7\end{array}$ & $\begin{array}{l}-- \\
-- \\
-- \\
-- \\
-- \\
-- \\
-- \\
-- \\
--\end{array}$ & $\begin{array}{l}\mathrm{R} \\
\mathrm{R} \\
\mathrm{R} \\
\mathrm{S} \\
\mathrm{T} \\
\mathrm{S} \\
\mathrm{S} \\
\mathrm{S} \\
\mathrm{T}\end{array}$ \\
\hline 148 & 179 N14 E64 06AA1 & 390649114491201 & 390645 & 1144917 & 200 & 6,693 & $\begin{array}{l}11 / 01 / 80 \\
01 / 01 / 81 \\
03 / 01 / 81 \\
03 / 03 / 81 \\
05 / 09 / 83 \\
10 / 04 / 84 \\
09 / 27 / 91\end{array}$ & $\begin{array}{l}132 \\
136 \\
135 \\
134.89 \\
135.1 \\
133.56 \\
131.77\end{array}$ & $\begin{array}{l}-- \\
-- \\
-- \\
-- \\
-- \\
-- \\
--\end{array}$ & $\begin{array}{l}\mathrm{R} \\
\mathrm{R} \\
\mathrm{R} \\
\mathrm{S} \\
\mathrm{T} \\
\mathrm{S} \\
\mathrm{S}\end{array}$ \\
\hline 149 & 179 N14 E64 14AA1 & 390500114444901 & 390500 & 1144449 & 167 & 6,759 & $\begin{array}{l}01 / 01 / 81 \\
03 / 01 / 81 \\
03 / 03 / 81 \\
09 / 27 / 91\end{array}$ & $\begin{array}{l}159 \\
159 \\
158.81 \\
155.03\end{array}$ & $\begin{array}{l}-- \\
-- \\
-- \\
--\end{array}$ & $\begin{array}{l}\mathrm{R} \\
\mathrm{R} \\
\mathrm{S} \\
\mathrm{S}\end{array}$ \\
\hline 150 & 179 N14 E64 15BD1 & 390442114462601 & 390446 & 1144624 & 150 & 6,644 & $\begin{array}{l}11 / 01 / 80 \\
01 / 01 / 81 \\
03 / 01 / 81 \\
03 / 03 / 81 \\
05 / 09 / 83 \\
08 / 22 / 83 \\
10 / 04 / 84 \\
09 / 27 / 91\end{array}$ & $\begin{array}{l}68.00 \\
51 \\
51 \\
50 \\
50.0 \\
49.78 \\
48.79 \\
--\end{array}$ & $\begin{array}{l}-- \\
-- \\
-- \\
-- \\
-- \\
-- \\
-- \\
0\end{array}$ & $\begin{array}{l}\mathrm{S} \\
\mathrm{R} \\
\mathrm{R} \\
\mathrm{R} \\
\mathrm{T} \\
\mathrm{S} \\
\mathrm{S} \\
--\end{array}$ \\
\hline 151 & 179 N14 E64 19DADA1 & 390337114491201 & 390337 & 1144905 & 198 & 6,720 & $\begin{array}{l}01 / 01 / 81 \\
03 / 03 / 81\end{array}$ & $\begin{array}{l}86 \\
86.99\end{array}$ & -- & $\begin{array}{l}\mathrm{R} \\
\mathrm{S}\end{array}$ \\
\hline 152 & 179 N15 E64 18BA1 & 391012114495801 & 391012 & 1144950 & 190 & 6,644 & $\begin{array}{c}01 / 01 / 81 \\
03 / 03 / 81 \\
05 / 05 / 83 \\
10 / 04 / 84 \\
09 / 25 / 91 \\
03 / 15 / 94\end{array}$ & $\begin{array}{l}64 \\
64 \\
68.4 \\
68.94 \\
70.78 \\
70.3\end{array}$ & $\begin{array}{l}-- \\
-- \\
-- \\
-- \\
-- \\
--\end{array}$ & $\begin{array}{l}\mathrm{R} \\
\mathrm{R} \\
\mathrm{T} \\
\mathrm{S} \\
\mathrm{S} \\
\mathrm{T}\end{array}$ \\
\hline
\end{tabular}


Table 1. Ground-water data for small-diameter monitoring wells - Continued

\begin{tabular}{|c|c|c|c|c|c|c|c|c|c|c|c|}
\hline \multirow[b]{2}{*}{$\begin{array}{c}\text { Map } \\
\text { no. } \\
\text { (pl. 1) }\end{array}$} & \multicolumn{3}{|c|}{ U.S. Geological Survey site designations } & \multirow[b]{2}{*}{ Latitude } & \multirow[b]{2}{*}{ Longitude } & \multirow[b]{2}{*}{$\begin{array}{l}\text { Well } \\
\text { depth } \\
\text { (feet) }\end{array}$} & \multirow{2}{*}{$\begin{array}{c}\text { Land- } \\
\text { surface } \\
\text { altitude } \\
\text { (feet above } \\
\text { sea level) }\end{array}$} & \multicolumn{4}{|c|}{ Water level } \\
\hline & & Local well number & $\begin{array}{c}\text { Standard } \\
\text { identification }\end{array}$ & & & & & Date $^{1}$ & $\begin{array}{c}\text { Feet } \\
\text { below } \\
\text { land } \\
\text { surface }^{2}\end{array}$ & $\begin{array}{c}\text { Site } \\
\text { status }\end{array}$ & Method \\
\hline \multirow[t]{6}{*}{153} & 179 & N15 E64 34DA1 & 390714114455201 & 390710 & 1144556 & 150 & 6,644 & $11 / 01 / 80$ & 78.00 & -- & $\mathrm{S}$ \\
\hline & & & & & & & & $01 / 01 / 81$ & 75 & -- & $\mathbf{R}$ \\
\hline & & & & & & & & $03 / 01 / 81$ & 76 & - & $\mathbf{R}$ \\
\hline & & & & & & & & $05 / 02 / 83$ & 74.90 & -- & $\mathrm{S}$ \\
\hline & & & & & & & & $10 / 04 / 84$ & 72.57 & -- & $\mathrm{S}$ \\
\hline & & & & & & & & $09 / 28 / 91$ & 76.09 & -- & $\mathrm{S}$ \\
\hline \multirow[t]{6}{*}{154} & 179 & N15 E64 35AC1 & 390712114451701 & 390724 & 1144503 & 200 & 6,759 & $01 / 01 / 80$ & 161 & -- & $\mathbf{R}$ \\
\hline & & & & & & & & $01 / 01 / 81$ & 161 & -- & $\mathbf{R}$ \\
\hline & & & & & & & & $03 / 01 / 81$ & 158 & -- & $\mathbf{R}$ \\
\hline & & & & & & & & $05 / 02 / 83$ & 157.8 & -- & $\mathrm{T}$ \\
\hline & & & & & & & & $10 / 04 / 84$ & 157.49 & -- & $\mathbf{S}$ \\
\hline & & & & & & & & $09 / 27 / 91$ & 153.94 & -- & $\mathrm{S}$ \\
\hline 155 & 180 & N09 E64 18AA1 & 383852114492601 & 383852 & 1144926 & 101 & 6,440 & $12 / 01 / 80$ & -- & $\mathrm{D}$ & -- \\
\hline 156 & 180 & N09 E64 20AD1 & 383746114482101 & 383746 & 1144821 & 200 & 6,100 & $08 / 19 / 93$ & -- & $\mathrm{Z}$ & -- \\
\hline 157 & 183 & N06 E65 25AA1 & 382123114371501 & 382123 & 1143715 & 200 & 6,040 & $03 / 01 / 81$ & -- & $\mathrm{D}$ & -- \\
\hline \multirow[t]{24}{*}{158} & 183 & N07 E66 16AC1 & 382753114341301 & 382810 & 1143418 & 97 & 5,915 & $07 / 01 / 80$ & 17 & -- & $\mathrm{R}$ \\
\hline & & & & & & & & $01 / 01 / 81$ & 17 & - & $\mathbf{R}$ \\
\hline & & & & & & & & $03 / 01 / 81$ & 17 & -- & $\mathbf{R}$ \\
\hline & & & & & & & & $11 / 14 / 91$ & 19.75 & -- & $\mathrm{S}$ \\
\hline & & & & & & & & $03 / 24 / 92$ & 19.72 & -- & $\mathrm{S}$ \\
\hline & & & & & & & & $06 / 22 / 92$ & 20.08 & -- & $\mathrm{S}$ \\
\hline & & & & & & & & $08 / 19 / 92$ & 19.53 & -- & $\mathrm{S}$ \\
\hline & & & & & & & & $09 / 15 / 92$ & 20.29 & -- & $\mathrm{S}$ \\
\hline & & & & & & & & $12 / 07 / 92$ & 20.29 & -- & $\mathrm{S}$ \\
\hline & & & & & & & & $04 / 06 / 93$ & 19.78 & -- & $\mathrm{S}$ \\
\hline & & & & & & & & $06 / 09 / 93$ & 19.83 & -- & $\mathrm{S}$ \\
\hline & & & & & & & & $06 / 16 / 93$ & 20.02 & -- & $\mathrm{S}$ \\
\hline & & & & & & & & $09 / 09 / 93$ & 20.09 & -- & $\mathrm{S}$ \\
\hline & & & & & & & & $12 / 09 / 93$ & 19.65 & -- & $S$ \\
\hline & & & & & & & & $03 / 30 / 94$ & 19.35 & -- & $\mathrm{S}$ \\
\hline & & & & & & & & $06 / 22 / 94$ & 19.56 & -- & $\mathrm{S}$ \\
\hline & & & & & & & & $09 / 22 / 94$ & 19.90 & -- & $\mathrm{S}$ \\
\hline & & & & & & & & $12 / 14 / 94$ & 20.03 & -- & $\mathrm{S}$ \\
\hline & & & & & & & & $03 / 29 / 95$ & 19.39 & -- & $\mathrm{S}$ \\
\hline & & & & & & & & $07 / 07 / 95$ & 19.02 & -- & $\mathrm{S}$ \\
\hline & & & & & & & & $10 / 17 / 95$ & 18.89 & -- & $\mathrm{S}$ \\
\hline & & & & & & & & $01 / 16 / 96$ & 19.10 & -- & $\mathrm{S}$ \\
\hline & & & & & & & & $03 / 19 / 96$ & 18.62 & -- & $\mathrm{S}$ \\
\hline & & & & & & & & $05 / 21 / 96$ & 18.72 & -- & $\mathrm{S}$ \\
\hline 159 & 183 & N08 E66 11AD1 & 383423114314601 & 383423 & 1143146 & 200 & 6,080 & 03/01/81 & -- & D & -- \\
\hline 160 & 183 & N08 E66 11BC1 & 383423114323601 & 383423 & 1143236 & 101 & 6,040 & $03 / 01 / 81$ & - & D & -- \\
\hline 161 & 183 & N08 E66 36CB1 & 383041114313101 & 383041 & 1143131 & 101 & 5,935 & $07 / 01 / 80$ & 85 & -- & $\mathbf{R}$ \\
\hline & & & & & & & & $01 / 01 / 81$ & 56 & -- & $\mathbf{R}$ \\
\hline 162 & 183 & N10 E66 20CA1 & 384245114360801 & 384245 & 1143608 & 160 & 5,990 & $01 / 01 / 81$ & -- & D & -- \\
\hline 163 & 183 & N10 E66 34CD1 & 38404811433500 & 384048 & 11433500 & 101 & 5,960 & $03 / 01 / 81$ & -- & $\mathrm{D}$ & -- \\
\hline 164 & 183 & N10 E66 34DC1 & 384048114333101 & 384048 & 1143331 & 101 & 6,100 & $01 / 01 / 81$ & - & D & -- \\
\hline 165 & 184 & N09 E68 21DC1 & 383720114205701 & 383720 & 1142057 & 96 & 5,930 & $03 / 01 / 81$ & -- & $\mathrm{D}$ & -- \\
\hline
\end{tabular}


Table 1. Ground-water data for small-diameter monitoring wells - Continued

\begin{tabular}{|c|c|c|c|c|c|c|c|c|c|c|c|}
\hline \multirow[b]{2}{*}{$\begin{array}{l}\text { Map } \\
\text { no. } \\
\text { (pl. 1) }\end{array}$} & \multicolumn{3}{|c|}{ U.S. Geological Survey site designations } & \multirow[b]{2}{*}{ Latitude } & \multirow[b]{2}{*}{ Longitude } & \multirow[b]{2}{*}{$\begin{array}{c}\text { Well } \\
\text { depth } \\
\text { (feet) }\end{array}$} & \multirow{2}{*}{$\begin{array}{c}\text { Land- } \\
\text { surface } \\
\text { altitude } \\
\text { (feet above } \\
\text { sea level) }\end{array}$} & \multicolumn{4}{|c|}{ Water level } \\
\hline & & Local well number & $\begin{array}{c}\text { Standard } \\
\text { identification }\end{array}$ & & & & & Date $^{1}$ & $\begin{array}{c}\text { Feet } \\
\text { below } \\
\text { land } \\
\text { surface }^{2}\end{array}$ & $\begin{array}{l}\text { Site } \\
\text { status }\end{array}$ & Method \\
\hline \multirow[t]{3}{*}{166} & 184 & N10 E67 07BA1 & 384448114300901 & 384448 & 1143009 & 200 & 5,880 & $07 / 01 / 80$ & 85 & -- & $\mathrm{R}$ \\
\hline & & & & & & & & 03/01/81 & 84 & -- & $\mathbf{R}$ \\
\hline & & & & & & & & $04 / 20 / 83$ & 85.50 & -- & $\mathrm{S}$ \\
\hline \multirow[t]{19}{*}{167} & 184 & N10 E67 22AA1 & 384310114261401 & 384310 & 1142614 & 100 & 5,889 & $07 / 01 / 80$ & 67 & -- & $\mathbf{R}$ \\
\hline & & & & & & & & $01 / 01 / 81$ & 70 & -- & $\mathbf{R}$ \\
\hline & & & & & & & & $03 / 01 / 81$ & 67 & -- & $\mathbf{R}$ \\
\hline & & & & & & & & $09 / 29 / 91$ & 65.58 & -- & $\mathrm{S}$ \\
\hline & & & & & & & & $03 / 24 / 92$ & 65.77 & -- & $\mathrm{S}$ \\
\hline & & & & & & & & $06 / 22 / 92$ & 65.75 & -- & $\mathrm{S}$ \\
\hline & & & & & & & & $09 / 15 / 92$ & 65.82 & -- & $\mathrm{S}$ \\
\hline & & & & & & & & $12 / 07 / 92$ & 65.78 & -- & $S$ \\
\hline & & & & & & & & $05 / 05 / 93$ & 66.02 & -- & $\mathrm{S}$ \\
\hline & & & & & & & & $03 / 30 / 94$ & 65.69 & - & $\mathrm{S}$ \\
\hline & & & & & & & & $06 / 15 / 94$ & 65.68 & -- & $\mathrm{S}$ \\
\hline & & & & & & & & $09 / 22 / 94$ & 65.73 & -- & $\mathrm{S}$ \\
\hline & & & & & & & & $12 / 14 / 94$ & 65.78 & -- & $\mathrm{S}$ \\
\hline & & & & & & & & $03 / 29 / 95$ & 65.84 & -- & $\mathrm{S}$ \\
\hline & & & & & & & & $07 / 06 / 95$ & 65.78 & -- & $\mathrm{S}$ \\
\hline & & & & & & & & $10 / 18 / 95$ & 65.30 & -- & $\mathrm{S}$ \\
\hline & & & & & & & & $01 / 07 / 96$ & 65.17 & -- & $\mathrm{S}$ \\
\hline & & & & & & & & $03 / 19 / 96$ & 65.00 & -- & $S$ \\
\hline & & & & & & & & $05 / 21 / 96$ & 64.94 & -- & $\mathrm{S}$ \\
\hline \multirow[t]{6}{*}{168} & 184 & N10 E67 26BB1 & 384216114260001 & 384216 & 1142600 & 200 & 5,944 & $07 / 01 / 80$ & 12 & - & $\mathrm{R}$ \\
\hline & & & & & & & & $01 / 01 / 81$ & 19 & -- & $\mathrm{R}$ \\
\hline & & & & & & & & $03 / 01 / 81$ & 17 & -- & $\mathrm{R}$ \\
\hline & & & & & & & & $04 / 20 / 83$ & 47.70 & -- & $\mathrm{S}$ \\
\hline & & & & & & & & $03 / 08 / 90$ & 87.2 & -- & $\mathrm{T}$ \\
\hline & & & & & & & & $09 / 29 / 91$ & 95.49 & -- & $S$ \\
\hline \multirow[t]{13}{*}{169} & 184 & N10 E68 31CD1 & 384039114232701 & 384039 & 1142327 & 150 & 5,906 & $07 / 01 / 80$ & 121 & -- & $\mathrm{R}$ \\
\hline & & & & & & & & $01 / 01 / 81$ & 121 & - & $\mathbf{R}$ \\
\hline & & & & & & & & $03 / 01 / 81$ & 120 & -- & $\mathbf{R}$ \\
\hline & & & & & & & & $04 / 20 / 83$ & 120.71 & -- & $\mathrm{S}$ \\
\hline & & & & & & & & $09 / 28 / 91$ & 119.59 & -- & $S$ \\
\hline & & & & & & & & $03 / 24 / 92$ & 119.56 & -- & $\mathrm{S}$ \\
\hline & & & & & & & & $06 / 22 / 92$ & 119.49 & -- & $\mathrm{S}$ \\
\hline & & & & & & & & $09 / 15 / 92$ & 119.44 & -- & $\mathrm{S}$ \\
\hline & & & & & & & & $12 / 07 / 92$ & 119.45 & - & $\mathrm{S}$ \\
\hline & & & & & & & & $05 / 05 / 93$ & 119.60 & -- & $\mathrm{S}$ \\
\hline & & & & & & & & $03 / 30 / 94$ & 119.44 & -- & $\mathrm{S}$ \\
\hline & & & & & & & & $06 / 15 / 94$ & 119.46 & -- & $\mathrm{S}$ \\
\hline & & & & & & & & $09 / 09 / 94$ & 119.37 & -- & $\mathrm{S}$ \\
\hline 170 & 184 & N11 E66 15CA1 & 384849114330701 & 384849 & 1143307 & 200 & 6,000 & $01 / 01 / 81$ & -- & $\mathrm{D}$ & -- \\
\hline \multirow[t]{5}{*}{171} & 184 & N11 E66 23AB1 & 384831114314301 & 384831 & 1143143 & 102 & 5,840 & $07 / 01 / 80$ & 47 & - & $\mathrm{R}$ \\
\hline & & & & & & & & $01 / 01 / 81$ & 48 & -- & $\mathbf{R}$ \\
\hline & & & & & & & & $03 / 01 / 81$ & 47 & -- & $\mathrm{R}$ \\
\hline & & & & & & & & $04 / 20 / 83$ & 48.00 & -- & $\mathrm{S}$ \\
\hline & & & & & & & & $03 / 08 / 90$ & 45.65 & -- & $\mathrm{S}$ \\
\hline
\end{tabular}


Table 1. Ground-water data for small-diameter monitoring wells - Continued

\begin{tabular}{|c|c|c|c|c|c|c|c|c|c|c|c|}
\hline \multirow[b]{2}{*}{$\begin{array}{l}\text { Map } \\
\text { no. } \\
\text { (pl. 1) }\end{array}$} & \multicolumn{3}{|c|}{ U.S. Geological Survey site designations } & \multirow[b]{2}{*}{ Latitude } & \multirow[b]{2}{*}{ Longitude } & \multirow[b]{2}{*}{$\begin{array}{c}\text { Well } \\
\text { depth } \\
\text { (feet) }\end{array}$} & \multirow{2}{*}{$\begin{array}{l}\text { Land- } \\
\text { surface } \\
\text { altitude } \\
\text { (feet above } \\
\text { sea level) }\end{array}$} & \multicolumn{4}{|c|}{ Water level } \\
\hline & & Local well number & $\begin{array}{c}\text { Standard } \\
\text { identification }\end{array}$ & & & & & Date $^{1}$ & $\begin{array}{c}\text { Feet } \\
\text { below } \\
\text { land } \\
\text { surface }^{2}\end{array}$ & $\begin{array}{l}\text { Site } \\
\text { status }\end{array}$ & Methoo \\
\hline \multirow[t]{3}{*}{171} & 184 & N11 E66 23AB1 & 384831114314301 & 384831 & 1143143 & 102 & 5,840 & $09 / 29 / 91$ & 46.77 & -- & $\mathrm{S}$ \\
\hline & & & & & & & & $09 / 17 / 92$ & 47.07 & -- & $\mathrm{S}$ \\
\hline & & & & & & & & $06 / 16 / 93$ & 47.86 & -- & $\mathrm{S}$ \\
\hline \multirow[t]{20}{*}{172} & 184 & N11 E68 19DCDC1 & 384745114224401 & 384741 & 1142243 & 200 & 5,906 & $01 / 01 / 81$ & 94 & -- & $\mathbf{R}$ \\
\hline & & & & & & & & $03 / 01 / 81$ & 94 & -- & $\mathbf{R}$ \\
\hline & & & & & & & & $04 / 19 / 83$ & 94.60 & -- & $\mathrm{S}$ \\
\hline & & & & & & & & $03 / 08 / 90$ & 93.1 & -- & $\mathrm{T}$ \\
\hline & & & & & & & & $09 / 28 / 91$ & 94.83 & -- & $\mathrm{S}$ \\
\hline & & & & & & & & $03 / 24 / 92$ & 95.20 & -- & $\mathrm{S}$ \\
\hline & & & & & & & & $06 / 22 / 92$ & 95.44 & -- & $\mathrm{S}$ \\
\hline & & & & & & & & $09 / 15 / 92$ & 95.80 & -- & $\mathrm{S}$ \\
\hline & & & & & & & & $12 / 07 / 92$ & 96.20 & -- & $\mathrm{S}$ \\
\hline & & & & & & & & $05 / 05 / 93$ & 96.60 & -- & $S$ \\
\hline & & & & & & & & $03 / 30 / 94$ & 97.43 & -- & $\mathrm{S}$ \\
\hline & & & & & & & & $06 / 15 / 94$ & 97.59 & -- & $\mathrm{S}$ \\
\hline & & & & & & & & $09 / 22 / 94$ & 97.68 & - & $S$ \\
\hline & & & & & & & & $12 / 13 / 94$ & 97.77 & -- & $\mathrm{S}$ \\
\hline & & & & & & & & $03 / 29 / 95$ & 97.98 & -- & $\mathrm{S}$ \\
\hline & & & & & & & & $07 / 06 / 95$ & 98.12 & -- & $\mathrm{S}$ \\
\hline & & & & & & & & $10 / 18 / 95$ & 98.09 & -- & $\mathrm{S}$ \\
\hline & & & & & & & & $01 / 17 / 96$ & 98.11 & -- & $\mathrm{S}$ \\
\hline & & & & & & & & $03 / 19 / 96$ & 97.97 & -- & $\mathrm{S}$ \\
\hline & & & & & & & & $05 / 21 / 96$ & 97.90 & -- & $\mathrm{S}$ \\
\hline \multirow[t]{2}{*}{173} & 184 & N13 E66 13CCAA1 & 385913114310401 & 385913 & 1143104 & 132 & 5,978 & $11 / 01 / 80$ & -- & D & -- \\
\hline & & & & & & & & $04 / 20 / 83$ & -- & D & -- \\
\hline \multirow[t]{4}{*}{174} & 184 & N13 E67 15DCDC1 & 385906114260501 & 385906 & 1142605 & 160 & 5,886 & $07 / 01 / 80$ & 90 . & -- & $\mathbf{R}$ \\
\hline & & & & & & & & $01 / 01 / 81$ & 94 & -- & $\mathbf{R}$ \\
\hline & & & & & & & & $03 / 01 / 81$ & 94 & -- & $\mathbf{R}$ \\
\hline & & & & & & & & $04 / 20 / 83$ & 95.11 & -- & $\mathrm{S}$ \\
\hline \multirow[t]{20}{*}{175} & 184 & N14 E66 24BDDD1 & 390352114305401 & 390352 & .1143054 & 160 & 5,840 & $01 / 01 / 81$ & 39 & -- & $\mathbf{R}$ \\
\hline & & & & & & & & $03 / 01 / 81$ & 39 & -- & $\mathbf{R}$ \\
\hline & & & & & & & & $04 / 21 / 83$ & 38.92 & -- & $\mathrm{S}$ \\
\hline & & & & & & & & $09 / 28 / 91$ & 35.94 & -- & $\mathrm{S}$ \\
\hline & & & & & & & & $03 / 17 / 92$ & 35.92 & -- & $\mathrm{S}$ \\
\hline & & & & & & & & $06 / 23 / 92$ & 36.05 & -- & $\mathrm{S}$ \\
\hline & & & & & & & & $09 / 16 / 92$ & 36.22 & -- & $\mathrm{S}$ \\
\hline & & & & & & & & $12 / 08 / 92$ & 37.01 & -- & $\mathrm{S}$ \\
\hline & & & & & & & & $04 / 07 / 93$ & 36.27 & -- & $\mathrm{S}$ \\
\hline & & & & & & & & $06 / 15 / 93$ & 36.25 & -- & $\mathrm{T}$ \\
\hline & & & & & & & & $06 / 22 / 93$ & 36.32 & -- & $\mathrm{S}$ \\
\hline & & & & & & & & $09 / 21 / 93$ & 36.44 & -- & $S$ \\
\hline & & & & & & & & $12 / 20 / 93$ & 36.57 & -- & $\mathrm{S}$ \\
\hline & & & & & & & & $03 / 25 / 94$ & 36.59 & -- & $\mathrm{S}$ \\
\hline & & & & & & & & $06 / 14 / 94$ & 36.63 & -- & $\mathrm{S}$ \\
\hline & & & & & & & & 09/07/94 & 36.77 & -- & $\mathrm{S}$ \\
\hline & & & & & & & & $12 / 13 / 94$ & 36.60 & -- & $\mathrm{S}$ \\
\hline & & & & & & & & $03 / 29 / 95$ & 36.95 & -- & $\mathrm{S}$ \\
\hline & & & & & & & & $07 / 06 / 95$ & 37.04 & -- & $\mathrm{S}$ \\
\hline & & & & & & & & $10 / 17 / 95$ & 37.20 & -- & $\mathrm{S}$ \\
\hline
\end{tabular}


Table 1. Ground-water data for small-diameter monitoring wells - Continued

\begin{tabular}{|c|c|c|c|c|c|c|c|c|c|c|c|}
\hline \multirow[b]{2}{*}{$\begin{array}{c}\text { Map } \\
\text { no. } \\
\text { (pl. 1) }\end{array}$} & \multicolumn{3}{|c|}{ U.S. Geological Survey site designations } & \multirow[b]{2}{*}{ Latitude } & \multirow[b]{2}{*}{ Longitude } & \multirow[b]{2}{*}{$\begin{array}{c}\text { Well } \\
\text { depth } \\
\text { (feet) }\end{array}$} & \multirow{2}{*}{$\begin{array}{l}\text { Land- } \\
\text { surface } \\
\text { altitude } \\
\text { (feet above } \\
\text { sea level) }\end{array}$} & \multicolumn{4}{|c|}{ Water level } \\
\hline & & Local well number & $\begin{array}{c}\text { Standard } \\
\text { identification }\end{array}$ & & & & & Date $^{1}$ & $\begin{array}{c}\text { Feet } \\
\text { below } \\
\text { land } \\
\text { surface }^{2}\end{array}$ & $\begin{array}{l}\text { Site } \\
\text { status }\end{array}$ & Method \\
\hline \multirow[t]{3}{*}{175} & 184 & N14 E66 24BDDD1 & 390352114305401 & 390352 & 1143054 & 160 & 5,840 & $01 / 17 / 96$ & 37.15 & -- & $\mathrm{S}$ \\
\hline & & & & & & & & $03 / 20 / 96$ & 37.26 & -- & $\mathrm{S}$ \\
\hline & & & & & & & & $05 / 21 / 96$ & 37.27 & -- & $\mathrm{S}$ \\
\hline \multirow[t]{4}{*}{176} & 184 & N15 E67 02DACB1 & 391135114244701 & 391135 & 1142447 & 185 & 5,780 & $07 / 01 / 80$ & 180 & -- & $\mathrm{R}$ \\
\hline & & & & & & & & $01 / 01 / 81$ & 151 & -- & $\mathrm{R}$ \\
\hline & & & & & & & & $03 / 01 / 81$ & 152 & -- & $\mathrm{R}$ \\
\hline & & & & & & & & $04 / 21 / 83$ & 150.44 & -- & $\mathrm{S}$ \\
\hline \multirow[t]{5}{*}{177} & 184 & N15 E67 26CA1 & 390803114251001 & 390803 & 1142527 & 200 & 5,676 & $01 / 01 / 81$ & 25 & - & $\mathrm{R}$ \\
\hline & & & & & & & & $04 / 21 / 83$ & 30.91 & -- & $\mathrm{S}$ \\
\hline & & & & & & & & $03 / 06 / 90$ & 31.9 & -- & $\mathrm{T}$ \\
\hline & & & & & & & & $09 / 28 / 91$ & 35.04 & -- & $\mathrm{S}$ \\
\hline & & & & & & & & $03 / 14 / 94$ & 37.3 & -- & $\mathrm{T}$ \\
\hline \multirow[t]{28}{*}{178} & 195 & N11 E70 35AD1 & 384702114041601 & 384702 & 1140416 & 101 & 5,578 & $01 / 01 / 81$ & 70 & -- & $\mathrm{R}$ \\
\hline & & & & & & & & $09 / 26 / 91$ & 69.28 & -- & $\mathrm{S}$ \\
\hline & & & & & & & & $03 / 25 / 92$ & 68.77 & -- & $\mathrm{S}$ \\
\hline & & & & & & & & $06 / 23 / 92$ & 69.00 & -- & $\mathrm{S}$ \\
\hline & & & & & & & & $09 / 16 / 92$ & 68.84 & -- & $\mathrm{S}$ \\
\hline & & & & & & & & $12 / 08 / 92$ & 68.75 & -- & $\mathrm{S}$ \\
\hline & & & & & & & & $04 / 06 / 93$ & 68.85 & -- & $\mathrm{S}$ \\
\hline & & & & & & & & $06 / 15 / 93$ & 68.79 & -- & $\mathrm{S}$ \\
\hline & & & & & & & & $06 / 29 / 93$ & 69.34 & -- & $\mathrm{S}$ \\
\hline & & & & & & & & $09 / 21 / 93$ & 69.07 & -- & $\mathrm{S}$ \\
\hline & & & & & & & & $12 / 20 / 93$ & 69.00 & -- & $\mathrm{S}$ \\
\hline & & & & & & & & $01 / 19 / 94$ & 69.28 & -- & $\mathrm{S}$ \\
\hline & & & & & & & & $03 / 24 / 94$ & 68.84 & -- & $\mathrm{S}$ \\
\hline & & & & & & & & $03 / 29 / 94$ & 68.8 & -- & $\mathrm{T}$ \\
\hline & & & & & & & & $05 / 11 / 94$ & 68.69 & - & $\mathrm{S}$ \\
\hline & & & & & & & & $06 / 14 / 94$ & 68.87 & -- & $\mathrm{S}$ \\
\hline & & & & & & & & $07 / 19 / 94$ & 68.83 & -- & $\mathrm{S}$ \\
\hline & & & & & & & & $09 / 07 / 94$ & 68.98 & -- & $\mathrm{S}$ \\
\hline & & & & & & & & $09 / 22 / 94$ & 68.93 & -- & $\mathrm{S}$ \\
\hline & & & & & & & & $11 / 04 / 94$ & 68.86 & -- & $\mathrm{S}$ \\
\hline & & & & & & & & $12 / 13 / 94$ & 68.95 & -- & $\mathrm{S}$ \\
\hline & & & & & & & & $03 / 29 / 95$ & 68.68 & -- & $\mathrm{S}$ \\
\hline & & & & & & & & $05 / 17 / 95$ & 68.8 & -- & $\mathrm{T}$ \\
\hline & & & & & & & & $07 / 06 / 95$ & 68.8 & -- & $\mathrm{T}$ \\
\hline & & & & & & & & $10 / 17 / 95$ & 69.0 & -- & $\mathrm{T}$ \\
\hline & & & & & & & & $01 / 17 / 96$ & 69.0 & -- & $\mathrm{T}$ \\
\hline & & & & & & & & $03 / 20 / 96$ & 68.8 & -- & $\mathrm{T}$ \\
\hline & & & & & & & & $05 / 21 / 96$ & 68.8 & -- & $\mathrm{T}$ \\
\hline \multirow[t]{5}{*}{179} & 195 & N11 E70 35BA1 & 384714114051001 & 384714 & 1140510 & 200 & 5,660 & $09 / 01 / 80$ & 143 & -- & $\mathrm{R}$ \\
\hline & & & & & & & & $01 / 01 / 81$ & 144 & -- & $\mathrm{R}$ \\
\hline & & & & & & & & $09 / 26 / 91$ & 141.08 & -- & $\mathrm{S}$ \\
\hline & & & & & & & & $03 / 25 / 92$ & 140.85 & -- & $\mathrm{S}$ \\
\hline & & & & & & & & $06 / 23 / 92$ & 141.09 & - & $\mathrm{S}$ \\
\hline
\end{tabular}


Table 1. Ground-water data for small-diameter monitoring wells - Continued

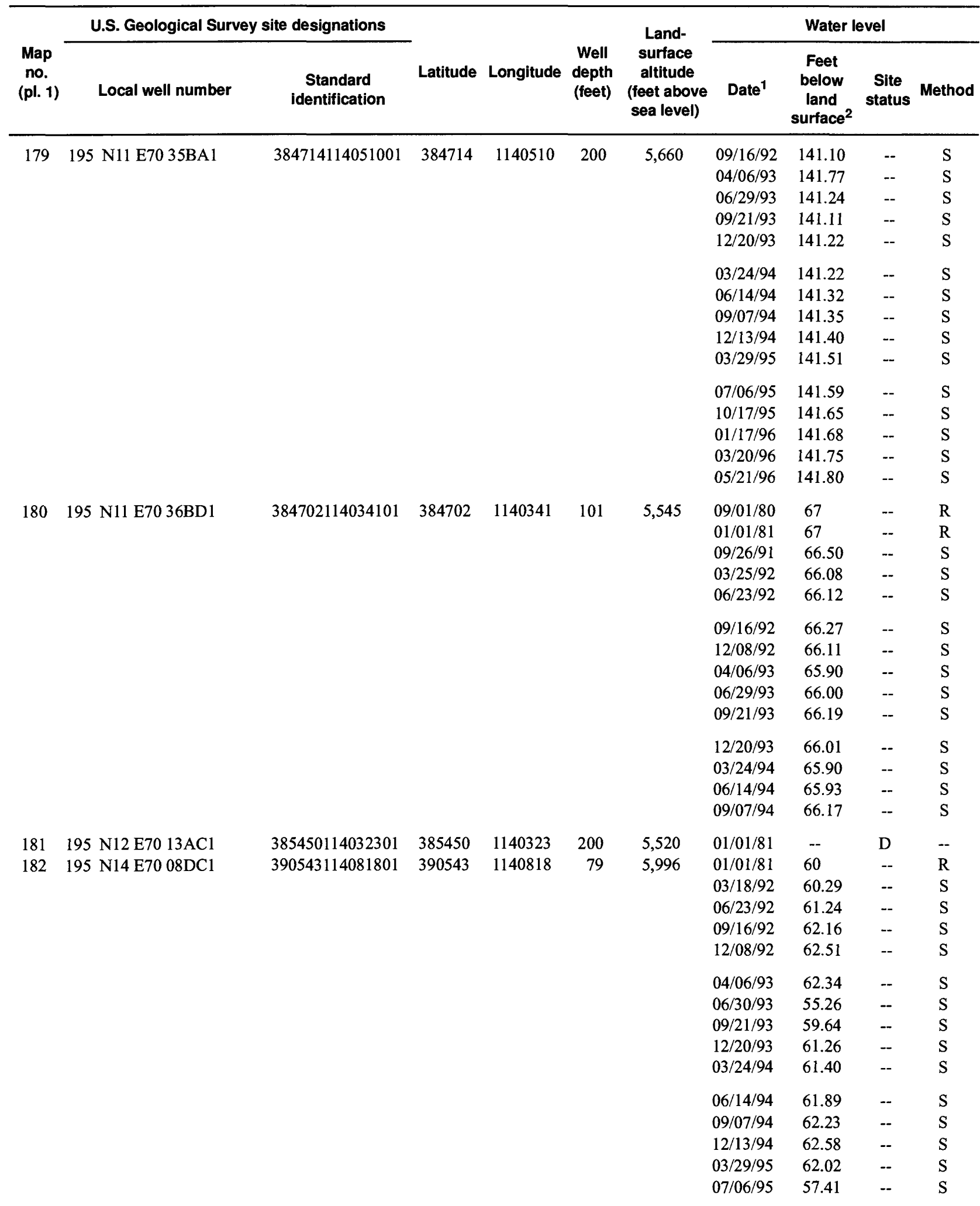


Table 1. Ground-water data for small-diameter monitoring wells - Continued

\begin{tabular}{|c|c|c|c|c|c|c|c|c|c|c|c|}
\hline \multirow[b]{2}{*}{$\begin{array}{l}\text { Map } \\
\text { no. } \\
\text { (pl. 1) }\end{array}$} & \multicolumn{3}{|c|}{ U.S. Geological Survey site designations } & \multirow[b]{2}{*}{ Latitude } & \multirow[b]{2}{*}{ Longitude } & \multirow[b]{2}{*}{$\begin{array}{l}\text { Well } \\
\text { depth } \\
\text { (feet) }\end{array}$} & \multirow{2}{*}{$\begin{array}{c}\text { Land- } \\
\text { surface } \\
\text { altitude } \\
\text { (feet above } \\
\text { sea level) }\end{array}$} & \multicolumn{4}{|c|}{ Water level } \\
\hline & & Local well number & $\begin{array}{c}\text { Standard } \\
\text { identification }\end{array}$ & & & & & Date $^{1}$ & $\begin{array}{c}\text { Feet } \\
\text { below } \\
\text { land } \\
\text { surface }^{2}\end{array}$ & $\begin{array}{l}\text { Site } \\
\text { status }\end{array}$ & Method \\
\hline \multirow[t]{4}{*}{182} & 195 & N14 E70 08DC1 & 390543114081801 & 390543 & 1140818 & 79 & 5,996 & $10 / 17 / 95$ & 57.04 & -- & $\mathrm{S}$ \\
\hline & & & & & & & & $01 / 17 / 96$ & 59.00 & -- & $\mathrm{S}$ \\
\hline & & & & & & & & $03 / 20 / 96$ & 59.89 & -- & $S$ \\
\hline & & & & & & & & $05 / 21 / 96$ & 57.38 & -- & $S$ \\
\hline \multirow[t]{21}{*}{183} & 195 & N15 E70 25DD1 & 390812114033601 & 390812 & 1140336 & 94 & 5,068 & $01 / 01 / 81$ & 14 & -- & $\mathrm{R}$ \\
\hline & & & & & & & & $03 / 01 / 81$ & 14 & -- & $\mathbf{R}$ \\
\hline & & & & & & & & $09 / 26 / 91$ & 10.63 & -- & $S$ \\
\hline & & & & & & & & $03 / 18 / 92$ & 10.57 & - & $\mathrm{S}$ \\
\hline & & & & & & & & $06 / 23 / 92$ & 11.03 & -- & $\mathrm{S}$ \\
\hline & & & & & & & & $09 / 16 / 92$ & 11.23 & -- & $\mathrm{S}$ \\
\hline & & & & & & & & $12 / 08 / 92$ & 11.16 & -- & $S$ \\
\hline & & & & & & & & $04 / 06 / 93$ & 11.00 & -- & $\mathrm{S}$ \\
\hline & & & & & & & & $06 / 29 / 93$ & 11.44 & -- & $\mathrm{S}$ \\
\hline & & & & & & & & $09 / 21 / 93$ & 11.70 & -- & $\mathrm{S}$ \\
\hline & & & & & & & & $12 / 20 / 93$ & 11.56 & - & $\mathrm{S}$ \\
\hline & & & & & & & & $03 / 24 / 94$ & 11.51 & -- & $\mathrm{S}$ \\
\hline & & & & & & & & $06 / 14 / 94$ & 11.91 & -- & $\mathrm{S}$ \\
\hline & & & & & & & & 09/07/94 & 12.26 & -- & $\mathrm{S}$ \\
\hline & & & & & & & & $12 / 13 / 94$ & 12.02 & -- & $\mathrm{S}$ \\
\hline & & & & & & & & $03 / 29 / 95$ & 12.05 & -- & $\mathrm{S}$ \\
\hline & & & & & & & & 07/06/95 & 12.26 & -- & $\mathrm{S}$ \\
\hline & & & & & & & & $10 / 17 / 95$ & 12.58 & -- & $\mathrm{S}$ \\
\hline & & & & & & & & $01 / 17 / 96$ & 12.55 & -- & $\mathrm{S}$ \\
\hline & & & & & & & & $03 / 20 / 96$ & 12.46 & -- & $\mathrm{S}$ \\
\hline & & & & & & & & $05 / 21 / 96$ & 12.60 & -- & $\mathrm{S}$ \\
\hline 184 & 196 & N08 E69 09DA1 & 383404114135201 & 383404 & 1141352 & 100 & 5,760 & $03 / 01 / 81$ & -- & $\mathrm{D}$ & - \\
\hline 185 & 196 & N09 E69 32DA1 & 383548114150101 & 383548 & 1141501 & 200 & 5,950 & $03 / 01 / 81$ & -- & $\mathrm{D}$ & -- \\
\hline \multirow[t]{2}{*}{186} & 196 & N09HE70 33ACl & 383559114072701 & 384108 & 1140739 & 101 & 5,650 & $07 / 01 / 80$ & 75 & -- & $\mathrm{R}$ \\
\hline & & & & & & & & $01 / 01 / 81$ & 77 & -- & $\mathrm{R}$ \\
\hline \multirow[t]{6}{*}{187} & 202 & N01 E67 08BAB1 & 375838114294601 & 375800 & 1142939 & 193 & 5,920 & $07 / 01 / 80$ & 184 & -- & $\mathrm{R}$ \\
\hline & & & & & & & & $01 / 01 / 81$ & -- & $\mathrm{D}$ & -- \\
\hline & & & & & & & & $03 / 04 / 81$ & 171 & -- & $\mathrm{R}$ \\
\hline & & & & & & & & $05 / 05 / 82$ & -- & $\mathrm{O}$ & -- \\
\hline & & & & & & & & $03 / 14 / 85$ & -- & D & -- \\
\hline & & & & & & & & $04 / 06 / 90$ & -- & $\mathrm{D}$ & -- \\
\hline 188 & 202 & N02 E66 13CA1 & 380152114314901 & 380152 & 1143149 & 200 & 5,920 & $01 / 01 / 81$ & -- & $\mathrm{D}$ & -- \\
\hline 189 & 202 & N02 E67 14AA1 & 380219114254701 & 380219 & 1142547 & 100 & 5,720 & $01 / 01 / 81$ & -- & $\mathrm{D}$ & -- \\
\hline 190 & 202 & N02 E67 18BC1 & 380206114305801 & 380206 & 1143058 & 100 & 5,800 & $01 / 01 / 81$ & -- & $\mathrm{D}$ & -- \\
\hline \multirow[t]{5}{*}{191} & 202 & N02 E67 35BCD1 & 375928114263501 & 375928 & 1142635 & 139 & 5,520 & $07 / 01 / 80$ & 56 & -- & $\mathrm{R}$ \\
\hline & & & & & & & & $01 / 01 / 81$ & 56 & -- & $\mathrm{R}$ \\
\hline & & & & & & & & $03 / 04 / 81$ & 56 & -- & $\mathrm{R}$ \\
\hline & & & & & & & & $03 / 14 / 85$ & 55.43 & -- & $\mathrm{S}$ \\
\hline & & & & & & & & $04 / 06 / 90$ & 64.0 & -- & $\mathrm{C}$ \\
\hline 192 & 202 & N02 E68 07BD1 & 380258114240801 & 380258 & 1142408 & 203 & 5,890 & $03 / 01 / 81$ & -- & $\mathrm{D}$ & -- \\
\hline \multirow[t]{2}{*}{193} & 202 & N03 E67 19BA1 & 380640114304101 & 380640 & 1143041 & 188 & 5,758 & $01 / 01 / 81$ & 147 & - & $\mathrm{R}$ \\
\hline & & & & & & & & $11 / 14 / 91$ & 44.52 & -- & $\mathrm{S}$ \\
\hline 194 & 207 & N04 E60 13AD1 & 381234115105601 & 381234 & 1151056 & 165 & 5,210 & $03 / 01 / 81$ & -- & $\mathrm{D}$ & -- \\
\hline
\end{tabular}


Table 1. Ground-water data for small-diameter monitoring wells - Continued

\begin{tabular}{|c|c|c|c|c|c|c|c|c|c|c|c|}
\hline \multirow[b]{2}{*}{$\begin{array}{l}\text { Map } \\
\text { no. } \\
\text { (pl. 1) }\end{array}$} & \multicolumn{3}{|c|}{ U.S. Geological Survey site designations } & \multirow[b]{2}{*}{ Latitude } & \multirow[b]{2}{*}{ Longitude } & \multirow[b]{2}{*}{$\begin{array}{l}\text { Well } \\
\text { depth } \\
\text { (feet) }\end{array}$} & \multirow{2}{*}{$\begin{array}{l}\text { Land- } \\
\text { surface } \\
\text { altitude } \\
\text { (feet above } \\
\text { sea level) }\end{array}$} & \multicolumn{4}{|c|}{ Water level } \\
\hline & & Local well number & $\begin{array}{c}\text { Standard } \\
\text { identification }\end{array}$ & & & & & Date $^{1}$ & $\begin{array}{c}\text { Feet } \\
\text { below } \\
\text { land } \\
\text { surface }^{2}\end{array}$ & $\begin{array}{c}\text { Site } \\
\text { status }\end{array}$ & Method \\
\hline \multirow[t]{7}{*}{195} & 207 & N05 E60 03ABBA1 & 381941115131801 & 381941 & 1151318 & 202 & 5,165 & $11 / 01 / 80$ & 48 & -- & $\mathrm{R}$ \\
\hline & & & & & & & & $09 / 05 / 91$ & 48.13 & -- & $\mathrm{S}$ \\
\hline & & & & & & & & $05 / 03 / 93$ & 48.00 & -- & $\mathrm{S}$ \\
\hline & & & & & & & & $12 / 16 / 93$ & 48.09 & -- & $\mathrm{S}$ \\
\hline & & & & & & & & $03 / 28 / 94$ & 48.08 & -- & $\mathrm{S}$ \\
\hline & & & & & & & & $07 / 06 / 94$ & 48.03 & -- & $\mathrm{S}$ \\
\hline & & & & & & & & $09 / 21 / 94$ & 47.99 & -- & $S$ \\
\hline 196 & 207 & N06 E60 19CA1 & 382151115164501 & 382151 & 1151645 & 200 & 5,360 & $12 / 01 / 80$ & -- & $\mathrm{D}$ & -- \\
\hline \multirow[t]{11}{*}{197} & 207 & N06 E61 27AADC1 & 382111115055901 & 382111 & 1150556 & 96 & 5,210 & $09 / 01 / 80$ & 78 & -- & $\mathrm{R}$ \\
\hline & & & & & & & & $01 / 01 / 81$ & 71 & -- & $\mathrm{R}$ \\
\hline & & & & & & & & $03 / 01 / 81$ & 71 & -- & $\mathrm{R}$ \\
\hline & & & & & & & & $03 / 13 / 85$ & 71.60 & -- & $\mathrm{S}$ \\
\hline & & & & & & & & $03 / 09 / 90$ & 69.9 & -- & $\mathrm{T}$ \\
\hline & & & & & & & & 09/05/91 & 70.87 & -- & $\mathrm{S}$ \\
\hline & & & & & & & & $05 / 03 / 93$ & 69.8 & -- & $\mathrm{T}$ \\
\hline & & & & & & & & $12 / 16 / 93$ & 69.74 & -- & $\mathbf{S}$ \\
\hline & & & & & & & & $03 / 28 / 94$ & 69.67 & -- & $\mathrm{S}$ \\
\hline & & & & & & & & 07/07/94 & 69.60 & -- & $\mathrm{S}$ \\
\hline & & & & & & & & $09 / 21 / 94$ & 69.53 & -- & $\mathrm{S}$ \\
\hline \multirow[t]{2}{*}{198} & 207 & N07 E61 04DDBA1 & 382942115070201 & 382927 & 1150702 & 162 & 5,245 & $01 / 01 / 81$ & 38 & -- & $\mathrm{R}$ \\
\hline & & & & & & & & $09 / 05 / 91$ & 38.03 & -- & $\mathrm{S}$ \\
\hline \multirow[t]{4}{*}{199} & 207 & N07 E61 19BDDC1 & 382718115094901 & 382705 & 1150949 & 101 & 5,245 & $11 / 01 / 80$ & 50 & -- & $\mathrm{R}$ \\
\hline & & & & & & & & $01 / 01 / 81$ & 49 & - & $\mathrm{R}$ \\
\hline & & & & & & & & $03 / 09 / 90$ & 48.5 & -- & $\mathrm{T}$ \\
\hline & & & & & & & & $09 / 04 / 91$ & 49.11 & -- & $\mathrm{S}$ \\
\hline \multirow[t]{4}{*}{200} & 207 & N08 E61 27DDBA1 & 383113115061201 & 383109 & 1150557 & 230 & 5,230 & $12 / 19 / 79$ & 43 & -- & $\mathrm{R}$ \\
\hline & & & & & & & & $03 / 22 / 90$ & 38.45 & -- & $\mathrm{S}$ \\
\hline & & & & & & & & $10 / 12 / 91$ & 39.66 & - & $\mathrm{S}$ \\
\hline & & & & & & & & $07 / 21 / 94$ & 39.81 & -- & $\mathrm{S}$ \\
\hline \multirow[t]{4}{*}{201} & 207 & N08 E61 27DDBA2 & 383113115061202 & 383109 & 1150557 & 410 & 5,230 & $12 / 19 / 79$ & 43 & -- & $\mathrm{R}$ \\
\hline & & & & & & & & $03 / 23 / 90$ & 38.24 & -- & $\mathrm{S}$ \\
\hline & & & & & & & & $10 / 12 / 91$ & 39.46 & -- & $\mathrm{S}$ \\
\hline & & & & & & & & $07 / 21 / 94$ & 39.60 & -- & $S$ \\
\hline 202 & 207 & N08 E62 28AD1 & 383139115002601 & 383139 & 1150026 & 198 & 5,530 & $01 / 01 / 81$ & -- & D & -- \\
\hline \multirow[t]{10}{*}{203} & 207 & N08 E62 30CD1 & 383133115030201 & 383109 & 1150312 & 101 & 5,285 & $09 / 01 / 80$ & 73 & - & $\mathrm{R}$ \\
\hline & & & & & & & & $01 / 01 / 81$ & 64 & -- & $\mathrm{R}$ \\
\hline & & & & & & & & $03 / 01 / 81$ & 65 & -- & $\mathrm{R}$ \\
\hline & & & & & & & & $03 / 22 / 90$ & 91.60 & -- & $\mathrm{S}$ \\
\hline & & & & & & & & $09 / 05 / 91$ & 27.72 & -- & $\mathrm{S}$ \\
\hline & & & & & & & & $10 / 12 / 91$ & 64.45 & -- & $\mathrm{S}$ \\
\hline & & & & & & & & $03 / 03 / 92$ & 64.31 & -- & $\mathrm{S}$ \\
\hline & & & & & & & & $03 / 27 / 92$ & 64.38 & -- & $\mathrm{S}$ \\
\hline & & & & & & & & $06 / 17 / 92$ & 64.39 & -- & $\mathrm{S}$ \\
\hline & & & & & & & & 09/09/92 & 64.42 & -- & $S$ \\
\hline
\end{tabular}


Table 1. Ground-water data for small-diameter monitoring wells - Continued

\begin{tabular}{|c|c|c|c|c|c|c|c|c|c|c|c|}
\hline \multirow[b]{2}{*}{$\begin{array}{c}\text { Map } \\
\text { no. } \\
\text { (pl. 1) }\end{array}$} & \multicolumn{3}{|c|}{ U.S. Geological Survey site designations } & \multirow[b]{2}{*}{ Latitude } & \multirow[b]{2}{*}{ Longitude } & \multirow[b]{2}{*}{$\begin{array}{c}\text { Well } \\
\text { depth } \\
\text { (feet) }\end{array}$} & \multirow{2}{*}{$\begin{array}{c}\text { Land- } \\
\text { surface } \\
\text { altitude } \\
\text { (feet above } \\
\text { sea level) }\end{array}$} & \multicolumn{4}{|c|}{ Water level } \\
\hline & & Local well number & $\begin{array}{c}\text { Standard } \\
\text { identification }\end{array}$ & & & & & Date $^{1}$ & $\begin{array}{c}\text { Feet } \\
\text { below } \\
\text { land } \\
\text { surface }^{2}\end{array}$ & $\begin{array}{l}\text { Site } \\
\text { status }\end{array}$ & Method \\
\hline \multirow[t]{9}{*}{203} & 207 & N08 E62 30CD1 & 383133115030201 & 383109 & 1150312 & 101 & 5,285 & $12 / 30 / 92$ & 64.59 & -- & $\mathrm{S}$ \\
\hline & & & & & & & & $04 / 01 / 93$ & 64.35 & -- & $\mathrm{S}$ \\
\hline & & & & & & & & $06 / 07 / 93$ & 64.40 & -- & $\mathrm{S}$ \\
\hline & & & & & & & & $09 / 08 / 93$ & 64.43 & -- & $\mathrm{S}$ \\
\hline & & & & & & & & $12 / 16 / 93$ & 64.52 & -- & $\mathrm{S}$ \\
\hline & & & & & & & & $03 / 29 / 94$ & 64.50 & -- & $\mathrm{S}$ \\
\hline & & & & & & & & $07 / 06 / 94$ & 64.50 & -- & $\mathrm{S}$ \\
\hline & & & & & & & & $07 / 21 / 94$ & 64.53 & -- & $\mathrm{S}$ \\
\hline & & & & & & & & $09 / 21 / 94$ & 64.56 & -- & $\mathrm{S}$ \\
\hline \multirow[t]{2}{*}{204} & 207 & N10 E60 01CC1 & 384502115111801 & 384502 & 1151118 & 197 & 5,496 & $11 / 01 / 80$ & 181 & -- & $\mathrm{R}$ \\
\hline & & & & & & & & $09 / 05 / 91$ & 178.27 & -- & $\mathrm{S}$ \\
\hline \multirow[t]{2}{*}{205} & 207 & N10 E60 33DA1 & 384060115134401 & 384109 & 1151344 & 200 & 5,453 & $11 / 01 / 80$ & 129 & -- & $\mathrm{R}$ \\
\hline & & & & & & & & $09 / 05 / 91$ & 127.69 & -- & $\mathrm{S}$ \\
\hline \multirow[t]{2}{*}{206} & 207 & N10 E61 13CD1 & 384323115041801 & 384323 & 1150418 & 51 & 5,380 & $10 / 01 / 80$ & 44 & -- & $\mathrm{R}$ \\
\hline & & & & & & & & $01 / 01 / 81$ & 42 & -- & $\mathrm{R}$ \\
\hline \multirow[t]{2}{*}{207} & 207 & N11 E61 27BA1 & 384729115062501 & 384729 & 1150625 & 161 & 5,437 & $01 / 01 / 81$ & 13 & -- & $\mathrm{R}$ \\
\hline & & & & & & & & $09 / 05 / 91$ & 12.15 & -- & $\mathrm{S}$ \\
\hline \multirow[t]{2}{*}{208} & 207 & N13 E62 32DA1 & 385636115012201 & 385636 & 1150122 & 200 & 5,709 & $01 / 01 / 81$ & 159 & -- & $\mathrm{R}$ \\
\hline & & & & & & & & $09 / 05 / 91$ & 157.85 & -- & $\mathrm{S}$ \\
\hline
\end{tabular}

\footnotetext{
${ }^{1}$ For some reported water levels, the exact date of measurement is not known. When only the year of measurement (for example, 1981) is known, the format "01/01/81" is used herein for reported water levels; likewise when only the month and year (for example, November 1980) are known, the format " $11 / 01 / 80 "$ is used.

${ }^{2}$ Water levels listed herein are from data base. Calibrated air-line and reported measurements are entered into the data base to the nearest foot; electric-tape measurements are entered to the nearest tenth of a foot; and steel-tape measurements are entered to the nearest hundredth of a foot.
} 
Table 2. Ground-water data for large-diameter production and observation wells

[Site status: D, site was dry; O, obstructed; P, well was being pumped; V, foreign substance present on surface of water; --, status unknown.

Water-level method: R, reported; S, steel tape; T, electric tape; --, measurement method unknown]

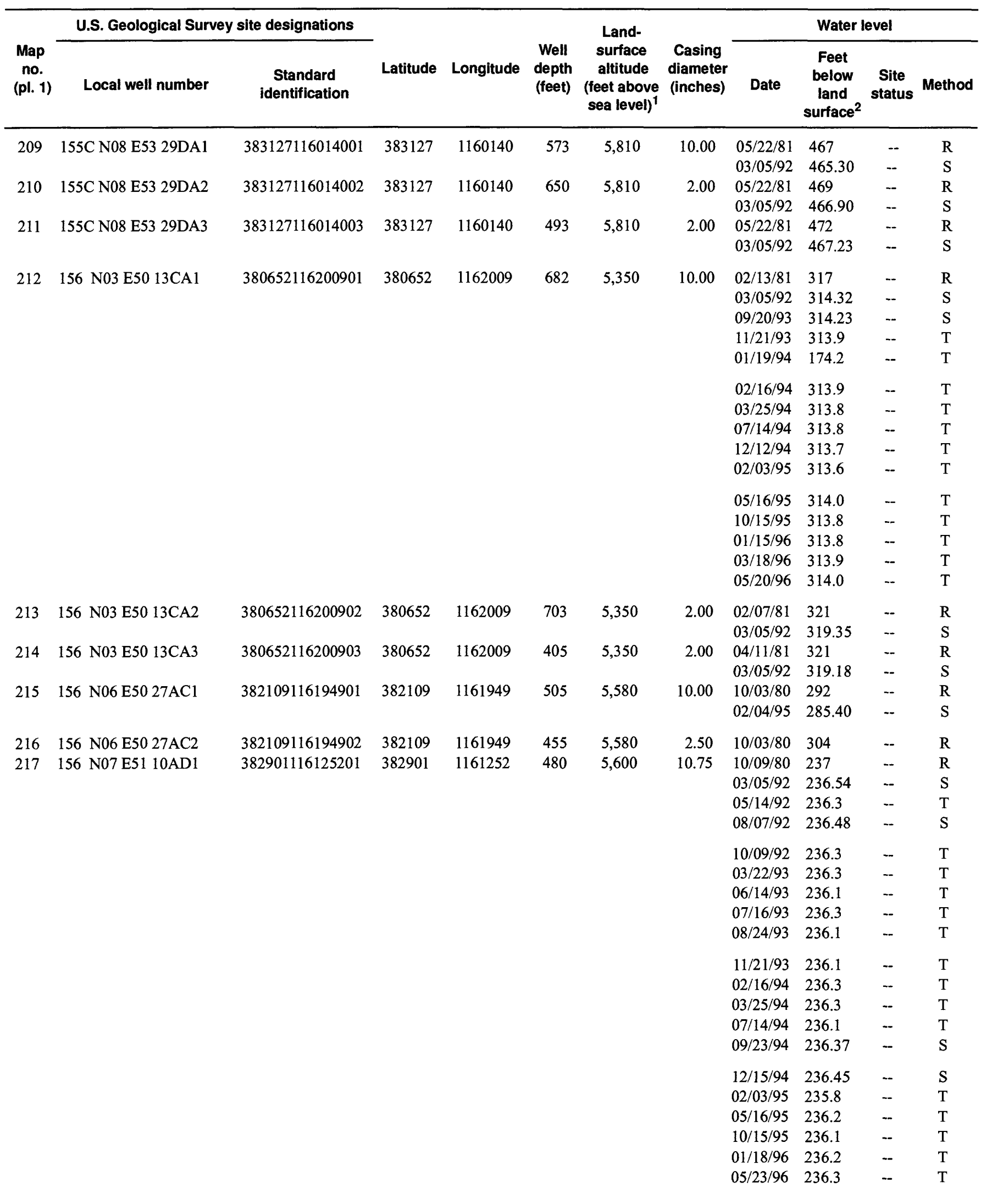


Table 2. Ground-water data for large-diameter production and observation wells - Continued

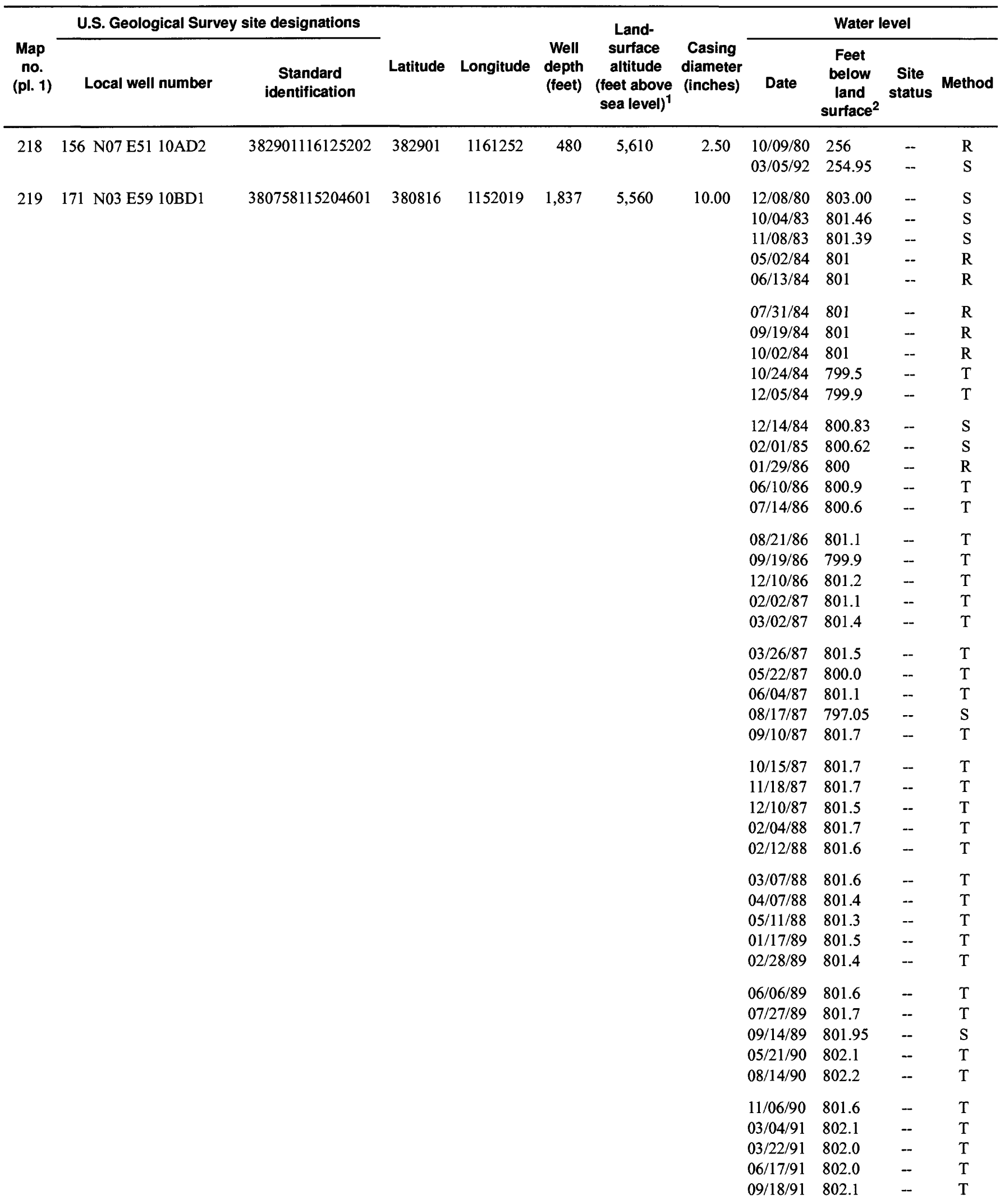


Table 2. Ground-water data for large-diameter production and observation wells - Continued

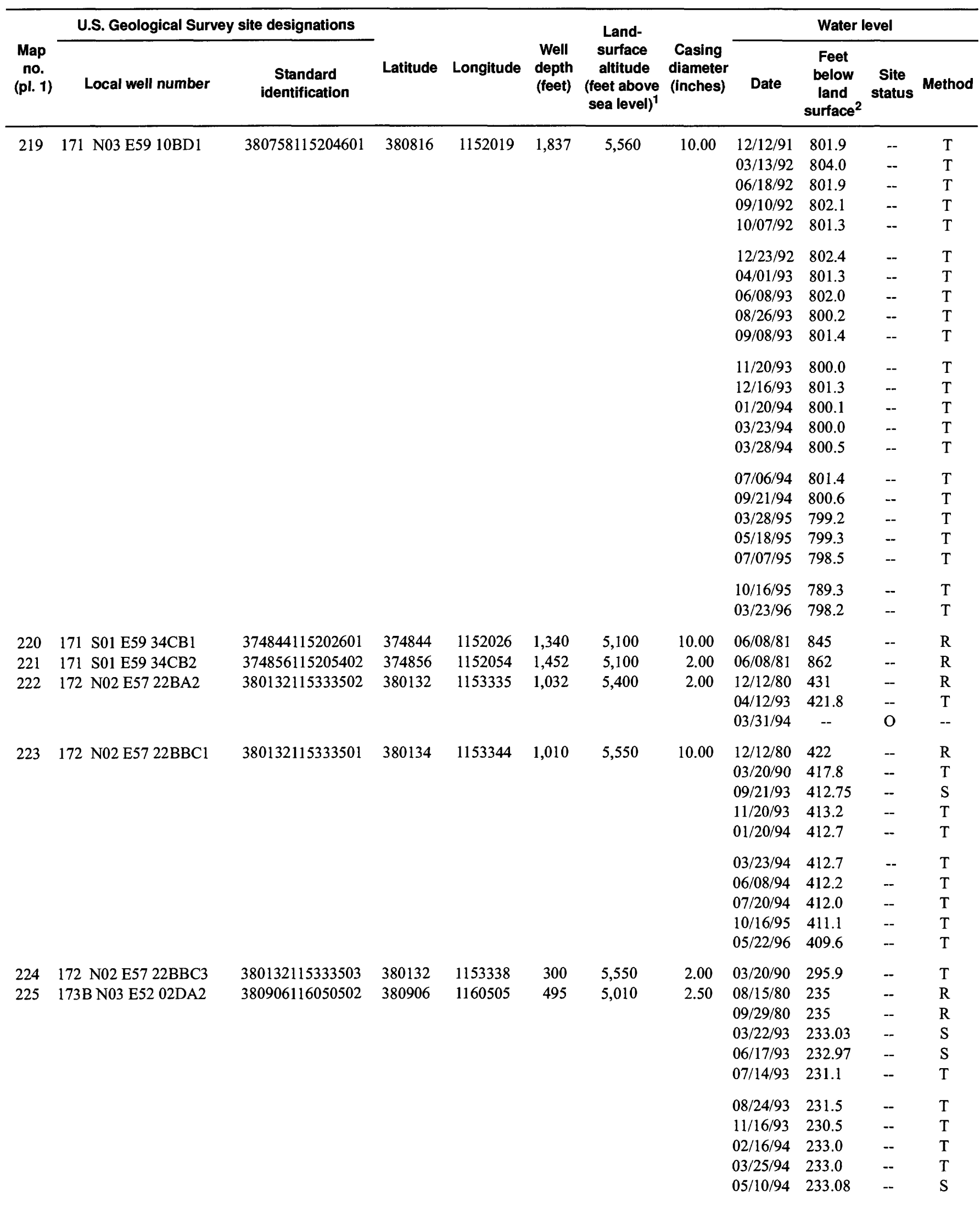


Table 2. Ground-water data for large-diameter production and observation wells - Continued

\begin{tabular}{|c|c|c|c|c|c|c|c|c|c|c|c|}
\hline \multirow{2}{*}{$\begin{array}{c}\text { Map } \\
\text { no. } \\
\text { (pl. 1) }\end{array}$} & \multicolumn{2}{|c|}{ U.S. Geological Survey site designations } & \multirow[b]{2}{*}{ Latitude } & \multirow[b]{2}{*}{ Longitude } & \multirow[b]{2}{*}{$\begin{array}{l}\text { Well } \\
\text { depth } \\
\text { (feet) }\end{array}$} & \multirow{2}{*}{$\begin{array}{c}\text { Land- } \\
\text { surface } \\
\text { altitude } \\
\text { (feet above } \\
\text { sea level) }\end{array}$} & \multirow[b]{2}{*}{$\begin{array}{l}\text { Casing } \\
\text { diameter } \\
\text { (inches) }\end{array}$} & \multicolumn{4}{|c|}{ Water level } \\
\hline & Local well number & $\begin{array}{l}\text { Standard } \\
\text { identification }\end{array}$ & & & & & & Date & $\begin{array}{c}\text { Feet } \\
\text { below } \\
\text { land } \\
\text { surface }\end{array}$ & $\begin{array}{c}\text { Site } \\
\text { status }\end{array}$ & Method \\
\hline \multirow[t]{8}{*}{225} & 173B N03 E52 02DA2 & 380906116050502 & 380906 & 1160505 & 495 & 5,010 & 2.50 & 07/14/94 & 232.8 & -- & $\mathrm{T}$ \\
\hline & & & & & & & & $12 / 12 / 94$ & 233.0 & -- & $\mathrm{T}$ \\
\hline & & & & & & & & $02 / 03 / 95$ & 232.4 & -- & $\mathrm{T}$ \\
\hline & & & & & & & & $05 / 16 / 95$ & 233.0 & -- & $\mathrm{T}$ \\
\hline & & & & & & & & $10 / 15 / 95$ & 232.8 & -- & $\mathrm{T}$ \\
\hline & & & & & & & & $01 / 15 / 96$ & 232.9 & -- & $\mathrm{T}$ \\
\hline & & & & & & & & 03/18/96 & 232.9 & -- & $\mathrm{T}$ \\
\hline & & & & & & & & $05 / 20 / 96$ & 232.8 & -- & $\mathrm{T}$ \\
\hline \multirow[t]{5}{*}{226} & 173B N03 E52 02DADB1 & 380906116050501 & 380906 & 1160505 & 452 & 5,005 & 10.00 & $08 / 25 / 80$ & 230 & - & $\mathbf{R}$ \\
\hline & & & & & & & & 09/29/80 & 233 & -- & $\mathbf{R}$ \\
\hline & & & & & & & & $05 / 14 / 84$ & 233.6 & -- & $\mathrm{T}$ \\
\hline & & & & & & & & $03 / 15 / 90$ & 231.6 & -- & $\mathrm{T}$ \\
\hline & & & & & & & & 07/16/91 & 231.4 & -- & $\mathrm{s}$ \\
\hline \multirow[t]{16}{*}{227} & 173B N10 E58 17BDDC1 & 384342115283501 & 384342 & 1152835 & 600 & 5,135 & 2.50 & $09 / 29 / 80$ & 282 & -- & $\mathrm{R}$ \\
\hline & & & & & & & & $06 / 06 / 84$ & 273.35 & - & $\mathrm{S}$ \\
\hline & & & & & & & & $03 / 06 / 90$ & 268.44 & -- & $\mathrm{S}$ \\
\hline & & & & & & & & 07/16/91 & 272.36 & -- & $\mathrm{s}$ \\
\hline & & & & & & & & $10 / 22 / 91$ & 272.74 & -- & $\mathrm{S}$ \\
\hline & & & & & & & & $12 / 05 / 91$ & 273.73 & -- & $\mathrm{s}$ \\
\hline & & & & & & & & $03 / 22 / 92$ & 274.91 & -- & $\mathrm{S}$ \\
\hline & & & & & & & & $06 / 24 / 92$ & 275.77 & - & $\mathrm{S}$ \\
\hline & & & & & & & & 09/17/92 & 276.72 & - & $\mathrm{S}$ \\
\hline & & & & & & & & $12 / 09 / 92$ & 277.61 & - & $\mathrm{S}$ \\
\hline & & & & & & & & 04/07/93 & 278.67 & - & $\mathrm{s}$ \\
\hline & & & & & & & & $06 / 30 / 93$ & 278.74 & - & $\mathrm{S}$ \\
\hline & & & & & & & & 09/16/93 & 278.92 & -- & $\mathrm{S}$ \\
\hline & & & & & & & & $\begin{array}{l}12 / 21 / 93 \\
03 / 26 / 94\end{array}$ & $\begin{array}{l}279.16 \\
276.9\end{array}$ & $\overline{-}$ & $\begin{array}{l}S \\
T\end{array}$ \\
\hline & & & & & & & & 07/07/94 & 277.68 & .- & $\mathrm{s}$ \\
\hline & & & & & & & & 09/20/94 & 278.20 & -- & $\mathrm{S}$ \\
\hline 228 & 173B N10 E58 17BDDC2 & 384342115283502 & 384342 & 1152835 & 220 & 5,135 & 2.50 & $09 / 21 / 80$ & -- & D & $\mathbf{R}$ \\
\hline \multirow[t]{14}{*}{229} & 173B N10 E58 17CAAB1 & 384338115283601 & 384338 & 1152836 & 581 & 5,135 & 10.00 & $09 / 29 / 80$ & 282 & -- & $\mathrm{R}$ \\
\hline & & & & & & & & 03/07/90 & 268.68 & -- & $\mathrm{S}$ \\
\hline & & & & & & & & $06 / 27 / 91$ & 291.56 & $\mathrm{~V}$ & $\mathrm{~S}$ \\
\hline & & & & & & & & $10 / 21 / 91$ & 288.88 & -- & $\mathrm{s}$ \\
\hline & & & & & & & & $12 / 05 / 91$ & 287.84 & -- & $\mathrm{S}$ \\
\hline & & & & & & & & $03 / 22 / 92$ & 284.57 & -- & $\mathrm{s}$ \\
\hline & & & & & & & & $05 / 12 / 92$ & 275.1 & -- & $\mathrm{T}$ \\
\hline & & & & & & & & $06 / 24 / 92$ & 281.40 & -- & $\mathrm{s}$ \\
\hline & & & & & & & & 08/18/92 & 276.07 & -- & $\mathrm{S}$ \\
\hline & & & & & & & & 09/05/92 & 276.53 & -- & $\mathrm{S}$ \\
\hline & & & & & & & & 09/17/92 & 279.97 & -- & $\mathrm{S}$ \\
\hline & & & & & & & & $12 / 09 / 92$ & 277.16 & -- & $\mathrm{S}$ \\
\hline & & & & & & & & 03/23/93 & 277.6 & -- & $\mathrm{T}$ \\
\hline & & & & & & & & $\begin{array}{l}04 / 07 / 93 \\
06 / 14 / 93\end{array}$ & $\begin{array}{l}279.07 \\
278.2\end{array}$ & -- & $\begin{array}{l}S \\
T\end{array}$ \\
\hline
\end{tabular}


Table 2. Ground-water data for large-diameter production and observation wells - Continued

\begin{tabular}{|c|c|c|c|c|c|c|c|c|c|c|c|}
\hline \multirow[b]{2}{*}{$\begin{array}{c}\text { Map } \\
\text { no. } \\
\text { (pl. 1) }\end{array}$} & \multicolumn{2}{|c|}{ U.S. Geological Survey site designations } & \multirow[b]{2}{*}{ Latitude } & \multirow[b]{2}{*}{ Longitude } & \multirow[b]{2}{*}{$\begin{array}{c}\text { Well } \\
\text { depth } \\
\text { (feet) }\end{array}$} & \multirow{2}{*}{$\begin{array}{c}\text { Land- } \\
\text { surface } \\
\text { altitude } \\
\text { (feet above } \\
\text { sea level) }\end{array}$} & \multirow{2}{*}{$\begin{array}{c}\text { Casing } \\
\text { diameter } \\
\text { (inches) }\end{array}$} & \multicolumn{4}{|c|}{ Water level } \\
\hline & Local well number & $\begin{array}{c}\text { Standard } \\
\text { identification }\end{array}$ & & & & & & Date & $\begin{array}{c}\text { Feet } \\
\text { below } \\
\text { land } \\
\text { surface }^{2}\end{array}$ & $\begin{array}{l}\text { Site } \\
\text { status }\end{array}$ & Method \\
\hline \multirow[t]{21}{*}{229} & 173B N10 E58 17CAAB1 & 384338115283601 & 384338 & 1152836 & 581 & 5,135 & 10.00 & $06 / 30 / 93$ & 278.37 & -- & $\mathrm{S}$ \\
\hline & & & & & & & & $07 / 14 / 93$ & 277.9 & -- & $\mathbf{T}$ \\
\hline & & & & & & & & $08 / 24 / 93$ & 278.0 & -- & $\mathrm{T}$ \\
\hline & & & & & & & & $09 / 16 / 93$ & 278.31 & -- & $\mathrm{S}$ \\
\hline & & & & & & & & $11 / 21 / 93$ & 278.4 & -- & $\mathbf{T}$ \\
\hline & & & & & & & & $12 / 21 / 93$ & 278.50 & - & $\mathrm{S}$ \\
\hline & & & & & & & & $02 / 17 / 94$ & 278.4 & -- & $\mathrm{T}$ \\
\hline & & & & & & & & $03 / 26 / 94$ & 277.1 & -- & $\mathrm{T}$ \\
\hline & & & & & & & & $06 / 07 / 94$ & 277.1 & - & $\mathrm{T}$ \\
\hline & & & & & & & & $07 / 07 / 94$ & 276.90 & -- & $\mathrm{S}$ \\
\hline & & & & & & & & $07 / 20 / 94$ & 277.3 & -- & $\mathrm{T}$ \\
\hline & & & & & & & & $09 / 20 / 94$ & 277.73 & -- & $S$ \\
\hline & & & & & & & & $09 / 23 / 94$ & 277.8 & -- & $\mathbf{T}$ \\
\hline & & & & & & & & $12 / 15 / 94$ & 278.36 & -- & $\mathrm{S}$ \\
\hline & & & & & & & & $02 / 03 / 95$ & 277.6 & - & $\mathrm{T}$ \\
\hline & & & & & & & & $05 / 16 / 95$ & 276.8 & -- & $\mathrm{T}$ \\
\hline & & & & & & & & $05 / 16 / 95$ & 276.8 & -- & $\mathrm{T}$ \\
\hline & & & & & & & & $10 / 15 / 95$ & 277.2 & -- & $\mathrm{T}$ \\
\hline & & & & & & & & $01 / 18 / 96$ & 275.9 & -- & $\mathrm{T}$ \\
\hline & & & & & & & & $03 / 21 / 96$ & 274.5 & -- & $\mathbf{T}$ \\
\hline & & & & & & & & $05 / 23 / 96$ & 274.4 & -- & $\mathrm{T}$ \\
\hline \multirow[t]{25}{*}{230} & 179 N12 E63 $12 \mathrm{AB} 1$ & 385521114503601 & 385521 & 1145036 & 640 & 7,320 & 6.00 & $10 / 13 / 80$ & 414 & -- & $\mathrm{R}$ \\
\hline & & & & & & & & $07 / 27 / 83$ & 414.38 & -- & $\mathrm{S}$ \\
\hline & & & & & & & & $08 / 15 / 83$ & 414.26 & -- & $\mathrm{S}$ \\
\hline & & & & & & & & $09 / 07 / 83$ & 413.18 & -- & $\mathrm{S}$ \\
\hline & & & & & & & & $09 / 15 / 83$ & 413.35 & -- & $\mathrm{S}$ \\
\hline & & & & & & & & $11 / 08 / 83$ & 414.36 & -- & $\mathrm{S}$ \\
\hline & & & & & & & & $12 / 30 / 83$ & 414.80 & -- & $\mathrm{S}$ \\
\hline & & & & & & & & $05 / 11 / 84$ & 413.62 & -- & $\mathrm{S}$ \\
\hline & & & & & & & & $05 / 15 / 84$ & 414.21 & -- & $\mathrm{S}$ \\
\hline & & & & & & & & $06 / 11 / 84$ & 410.40 & -- & $\mathrm{S}$ \\
\hline & & & & & & & & $07 / 31 / 84$ & 410.35 & -- & $\mathrm{S}$ \\
\hline & & & & & & & & $09 / 21 / 84$ & 411.38 & -- & $\mathrm{S}$ \\
\hline & & & & & & & & $10 / 15 / 84$ & 411.56 & -- & $\mathrm{S}$ \\
\hline & & & & & & & & $11 / 03 / 85$ & 412.25 & -- & $\mathrm{S}$ \\
\hline & & & & & & & & $04 / 24 / 86$ & 411.87 & -- & $\mathrm{S}$ \\
\hline & & & & & & & & $08 / 05 / 86$ & 409.72 & - & $\mathrm{S}$ \\
\hline & & & & & & & & $11 / 05 / 86$ & 410.78 & - & $\mathrm{S}$ \\
\hline & & & & & & & & $01 / 14 / 87$ & 411.80 & -- & $\mathrm{S}$ \\
\hline & & & & & & & & $02 / 12 / 87$ & 411.74 & - & $\mathrm{S}$ \\
\hline & & & & & & & & $03 / 25 / 87$ & 412.19 & -- & $\mathrm{S}$ \\
\hline & & & & & & & & $05 / 29 / 87$ & 413.07 & -- & $\mathrm{S}$ \\
\hline & & & & & & & & $07 / 23 / 87$ & 412.68 & -- & $\mathrm{S}$ \\
\hline & & & & & & & & $12 / 08 / 87$ & 415.18 & -- & $\mathrm{S}$ \\
\hline & & & & & & & & $01 / 20 / 88$ & 415.80 & - & $\mathrm{S}$ \\
\hline & & & & & & & & $05 / 18 / 88$ & 417.20 & -- & $\mathrm{S}$ \\
\hline
\end{tabular}


Table 2. Ground-water data for large-diameter production and observation wells - Continued

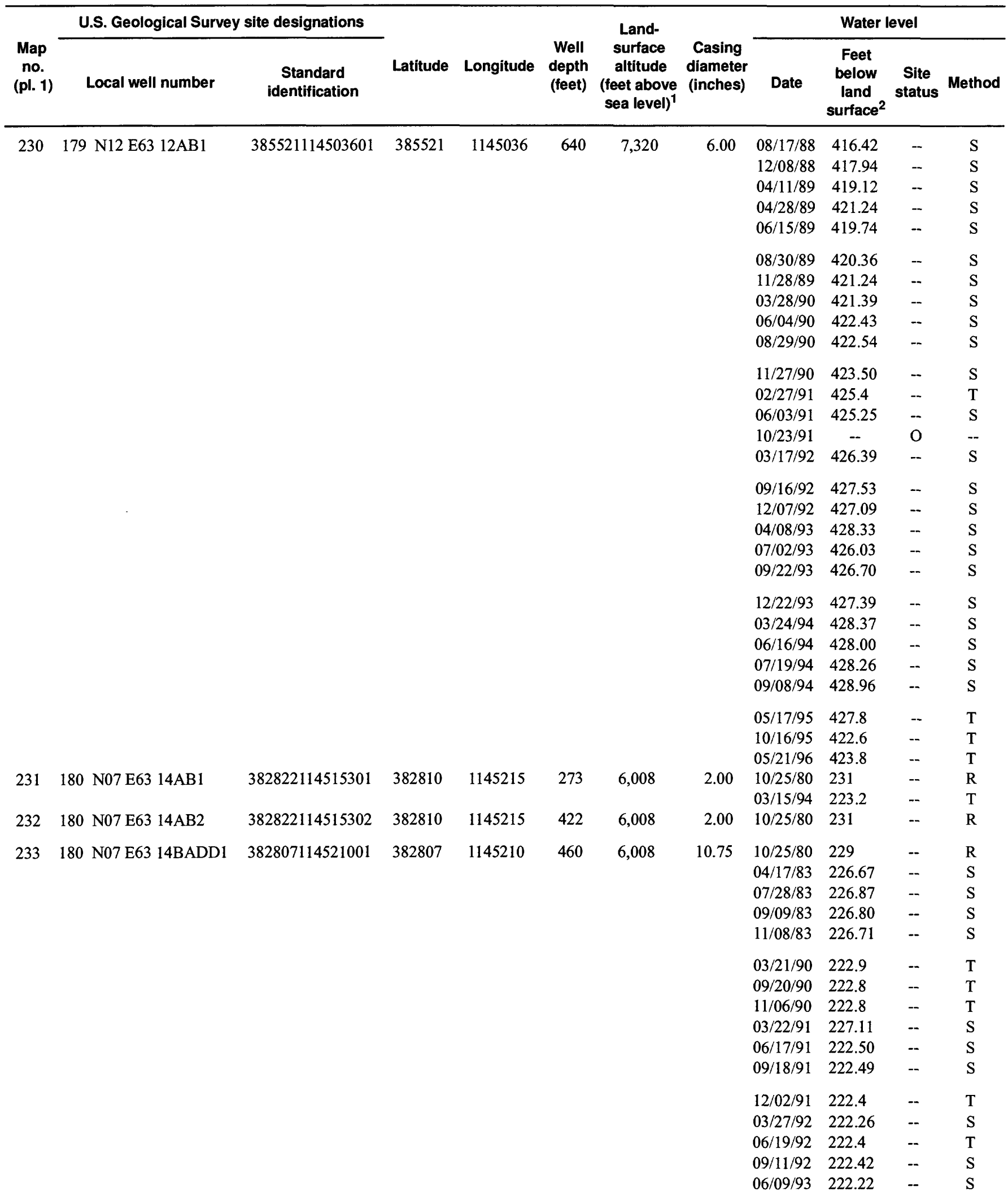


Table 2. Ground-water data for large-diameter production and observation wells -Continued

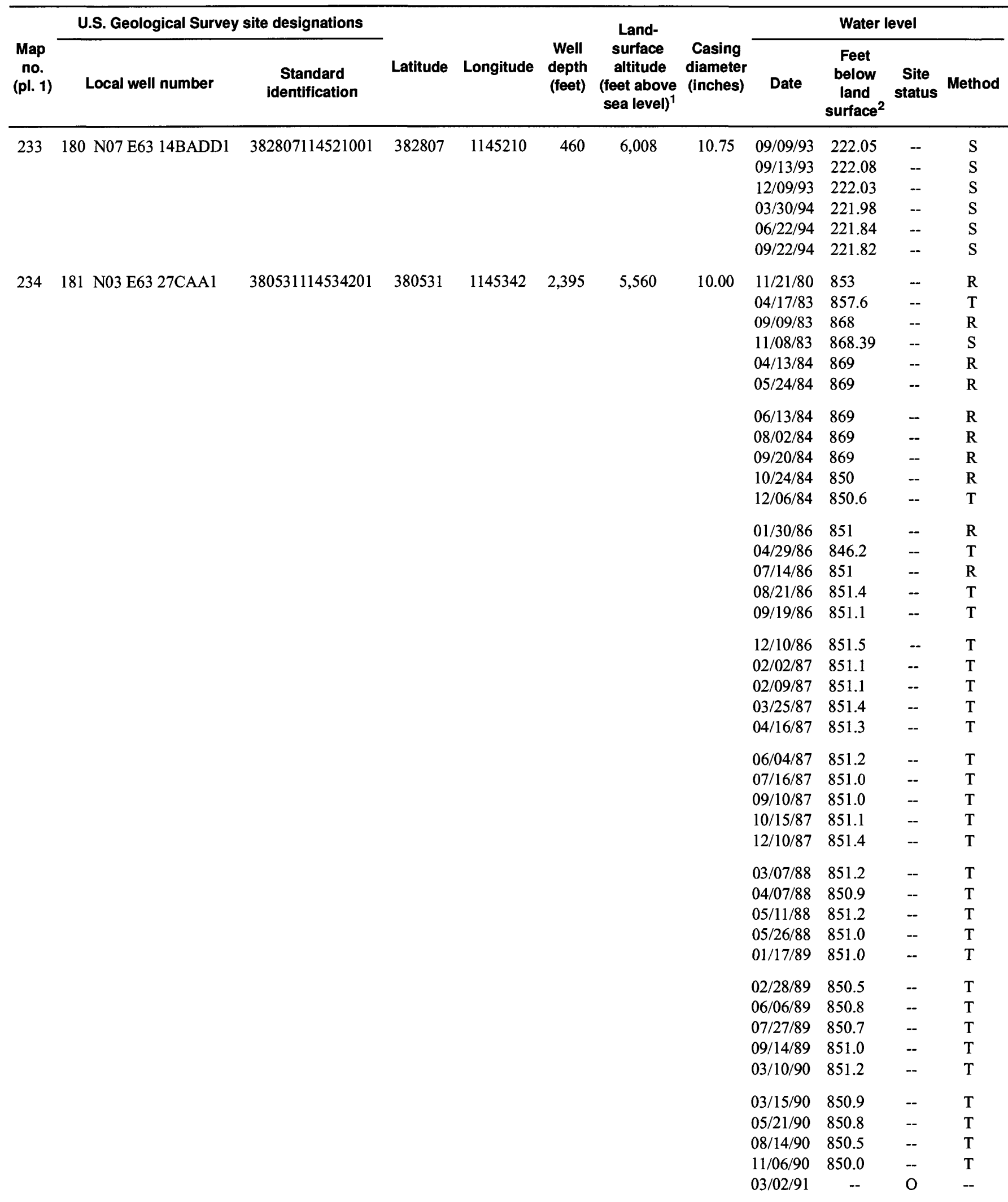


Table 2. Ground-water data for large-diameter production and observation wells - Continued

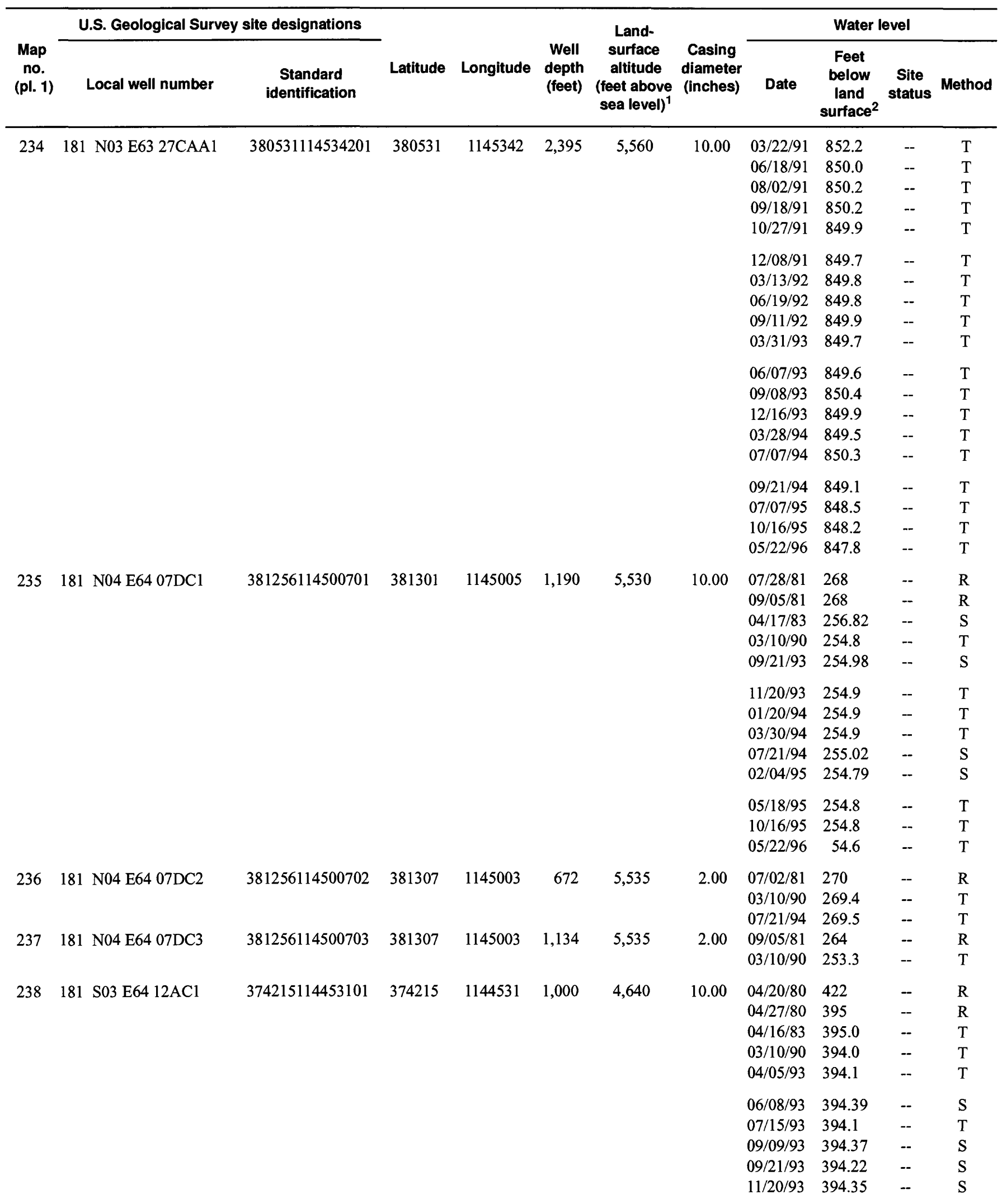


Table 2. Ground-water data for large-diameter production and observation wells - Continued

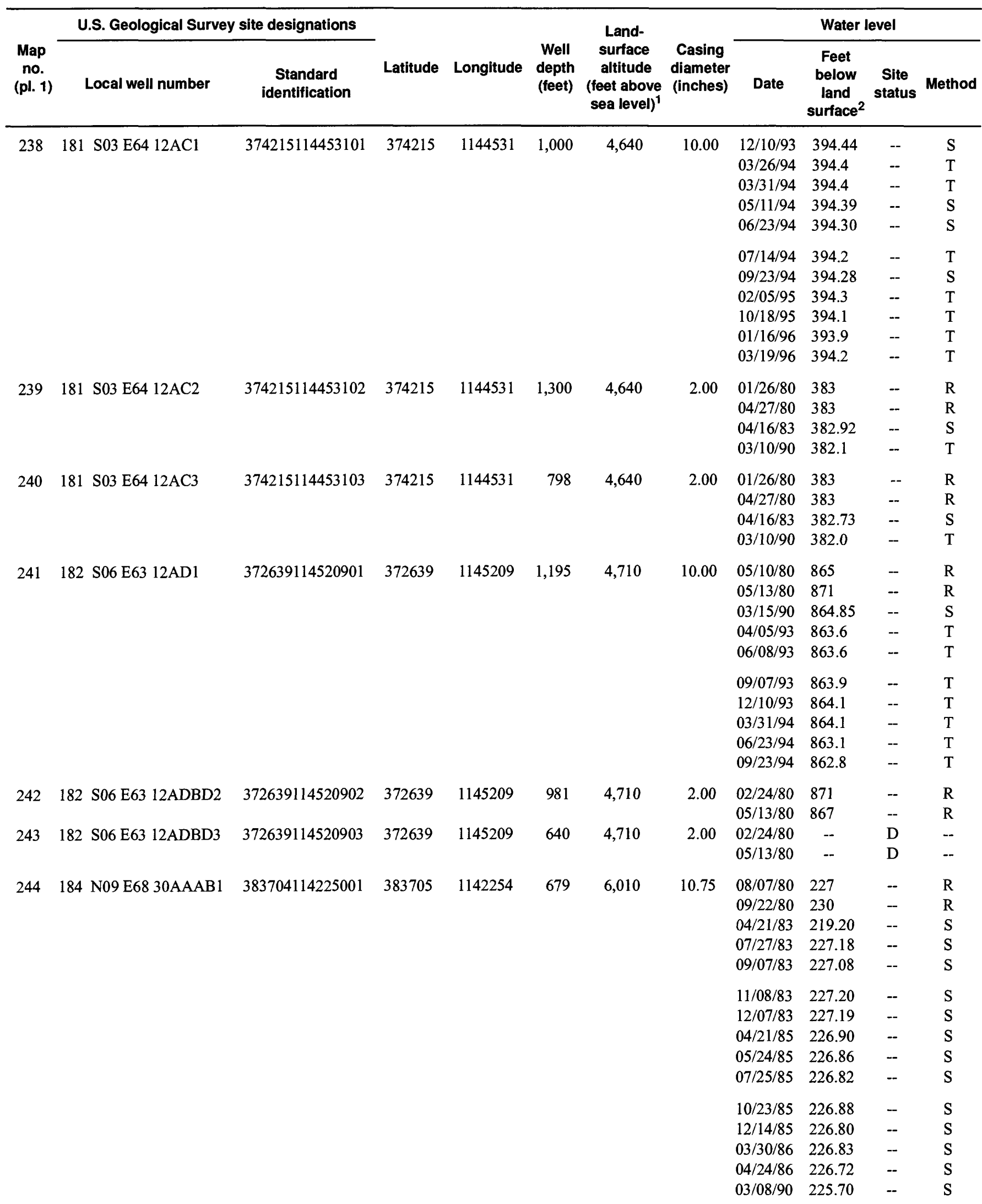


Table 2. Ground-water data for large-diameter production and observation wells -Continued

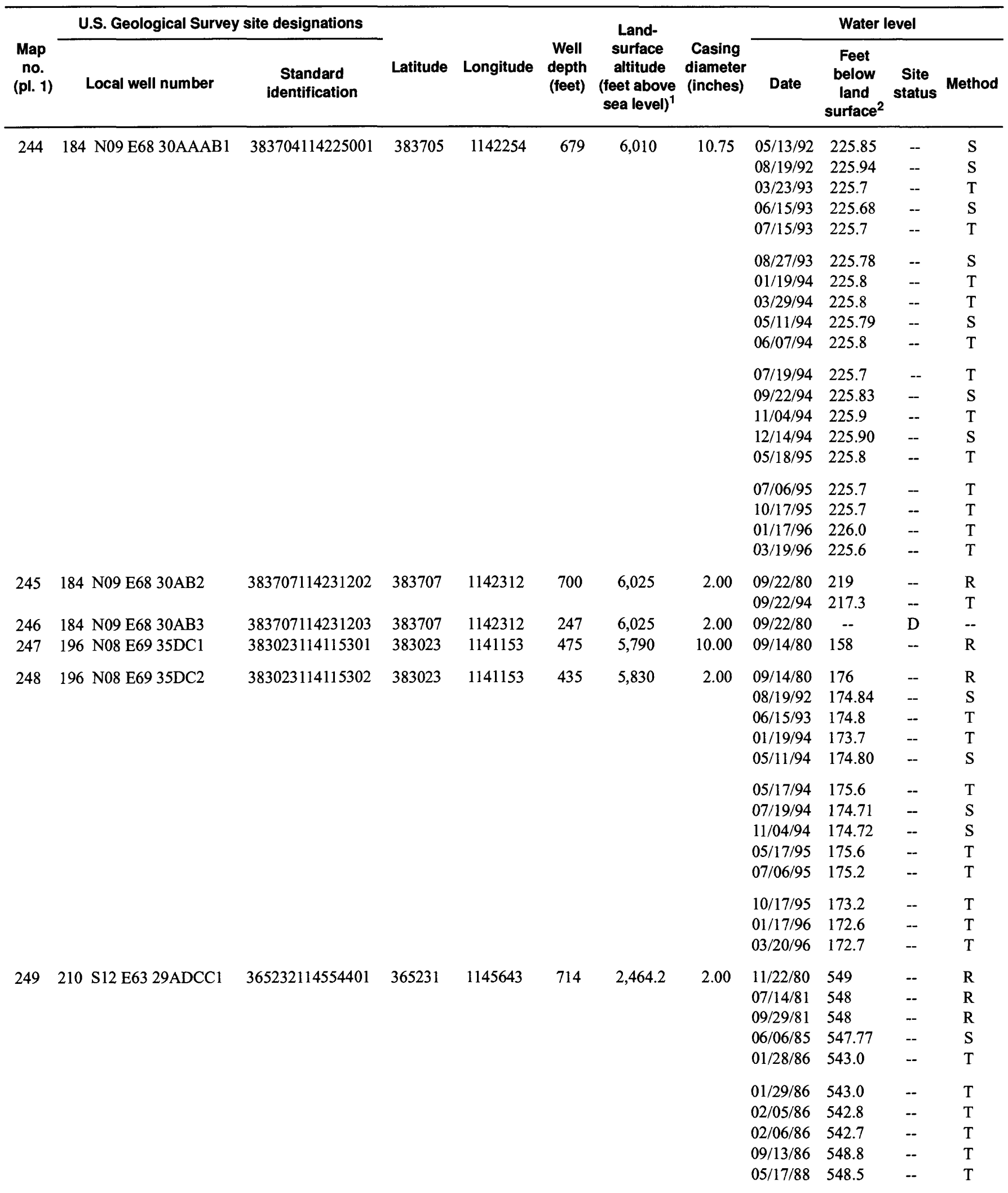


Table 2. Ground-water data for large-diameter production and observation wells - Continued

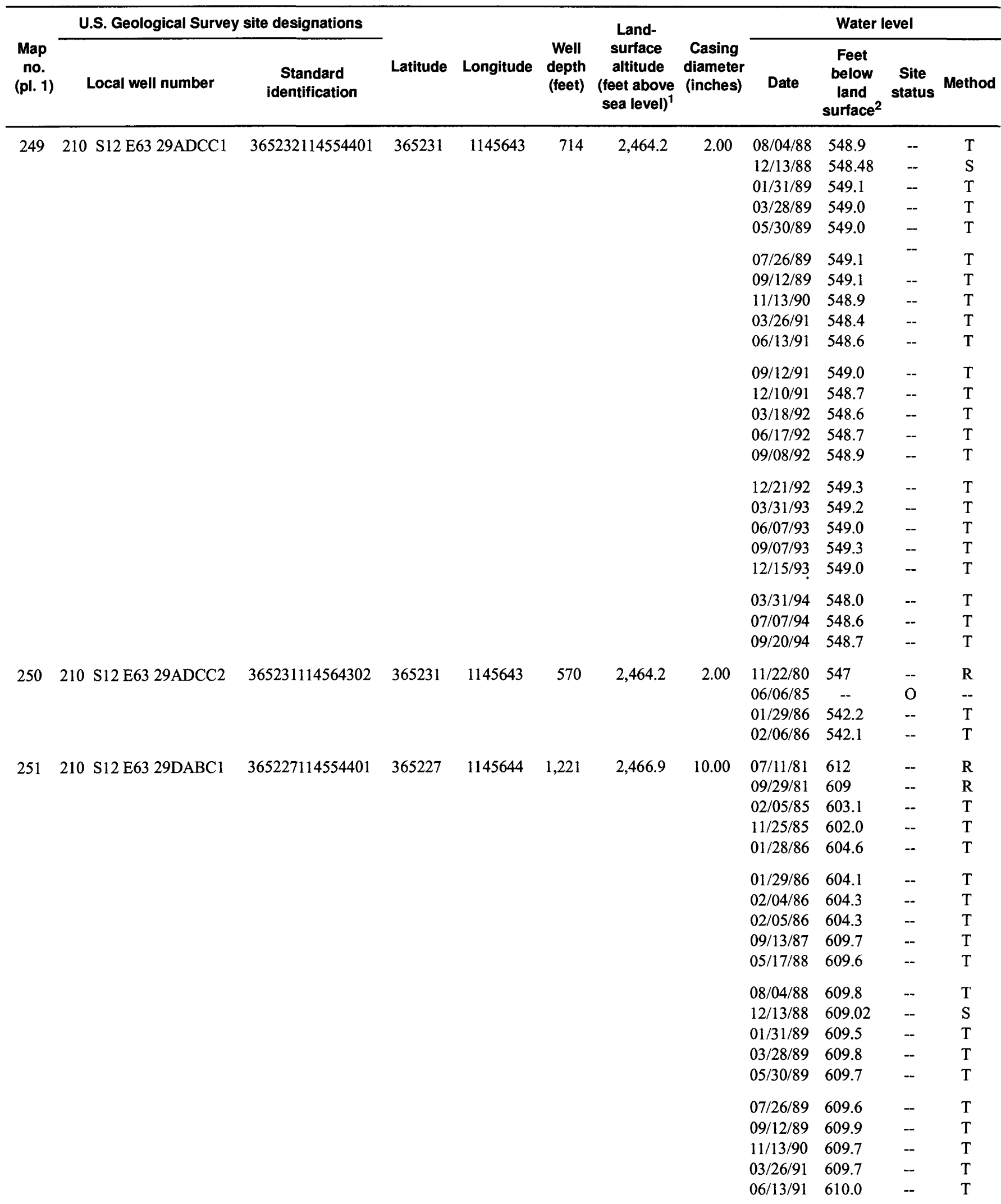


Table 2. Ground-water data for large-diameter production and observation wells - Continued

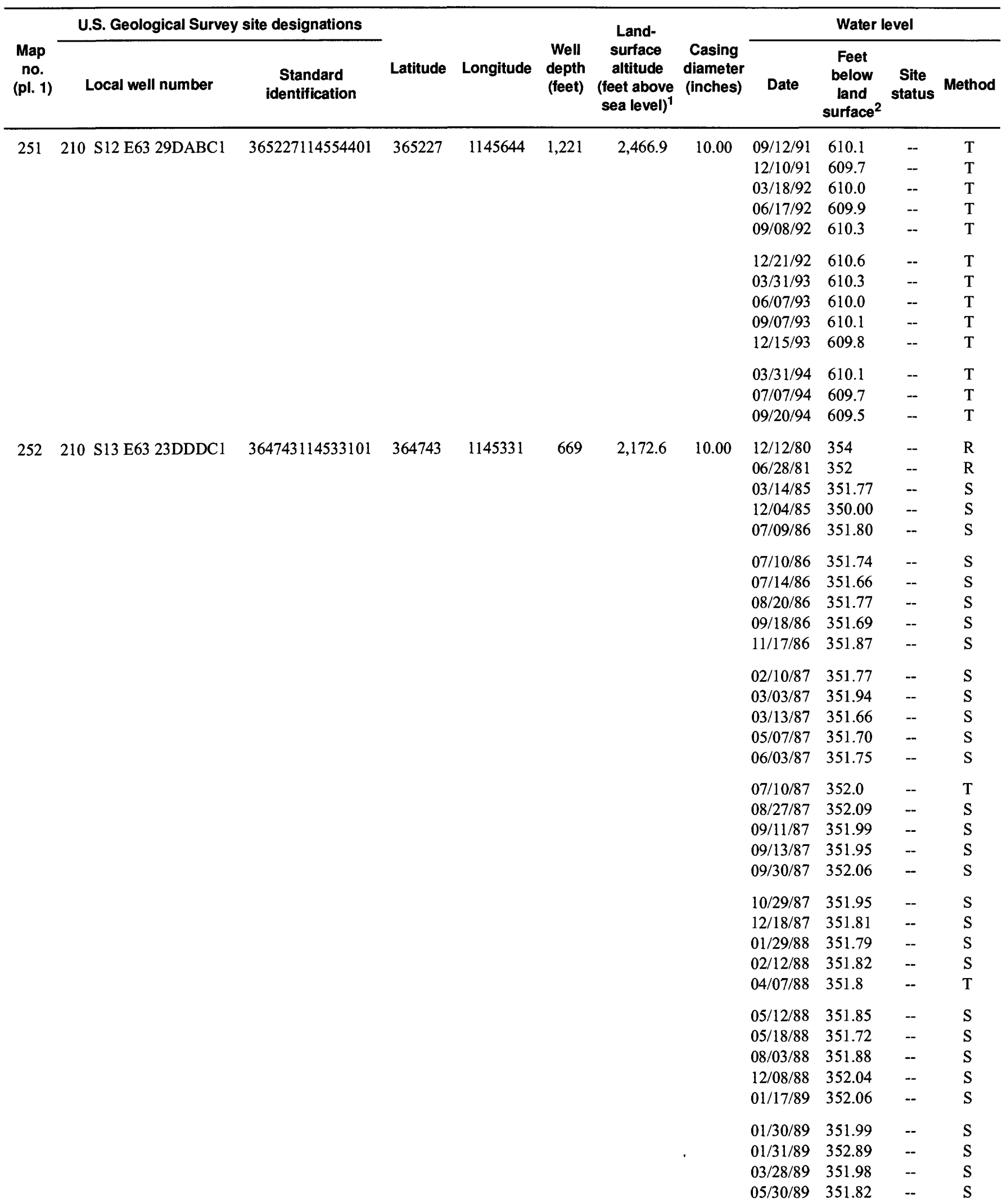


Table 2. Ground-water data for large-diameter production and observation wells - Continued

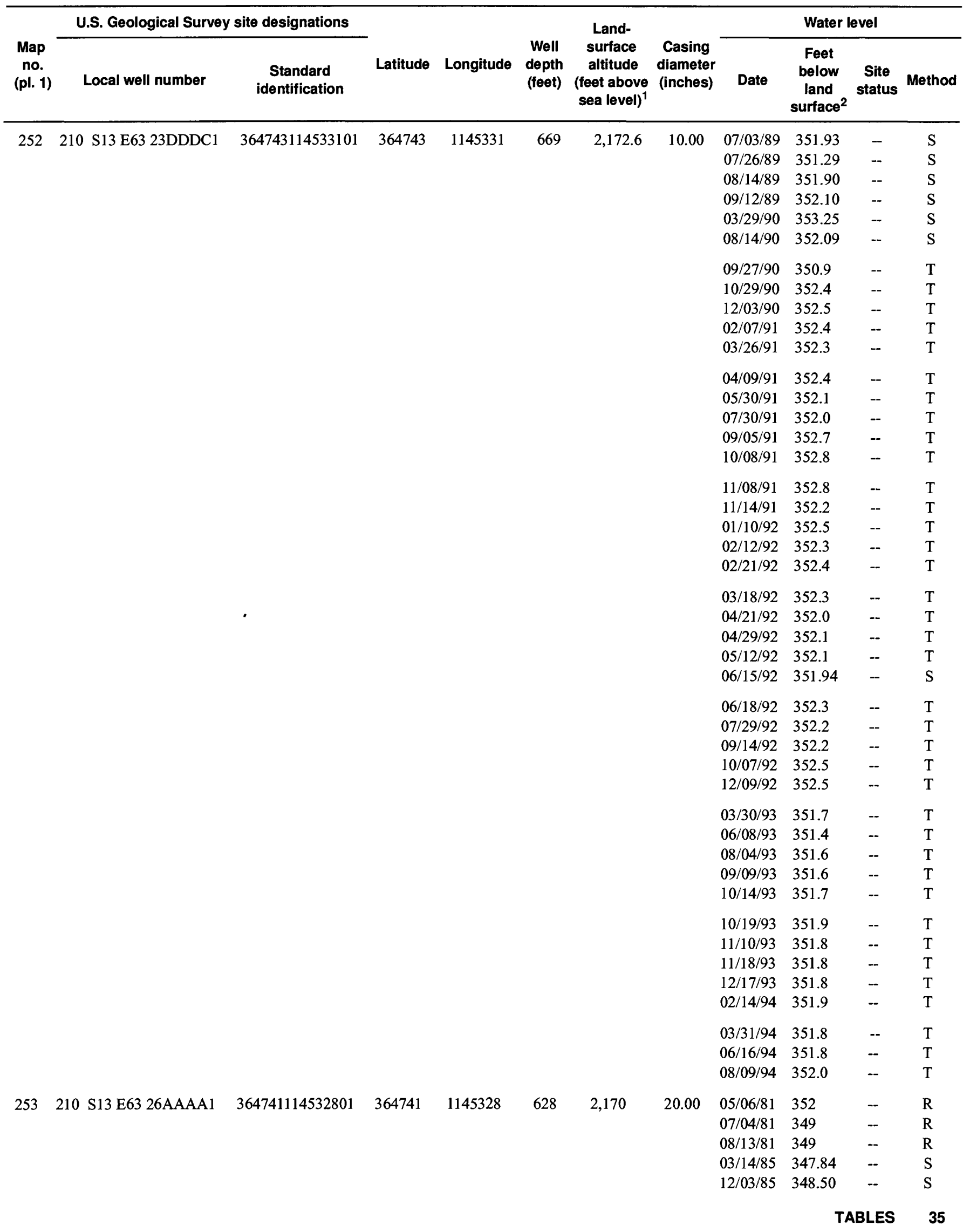


Table 2. Ground-water data for large-diameter production and observation wells - Continued

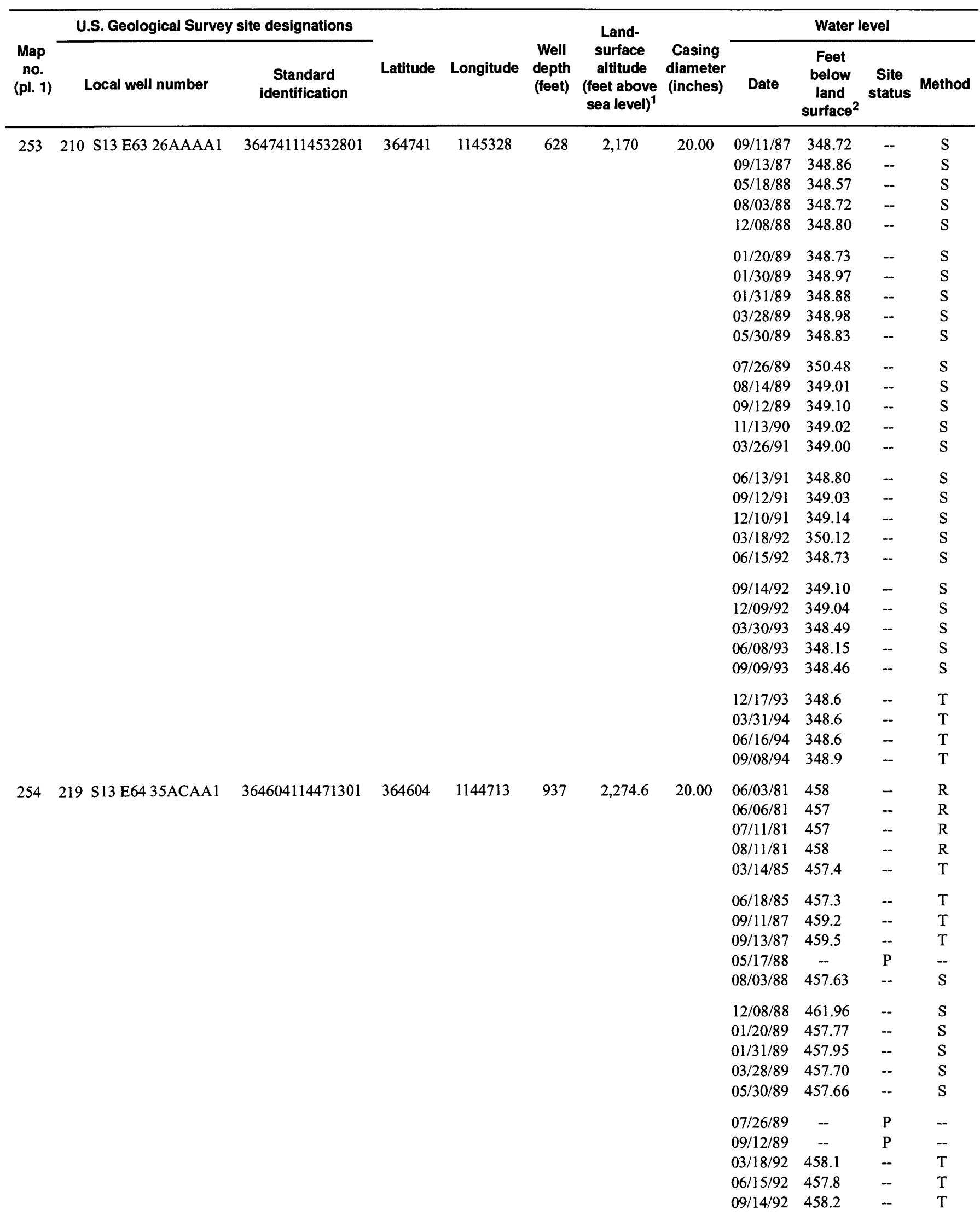


Table 2. Ground-water data for large-diameter production and observation wells - Continued

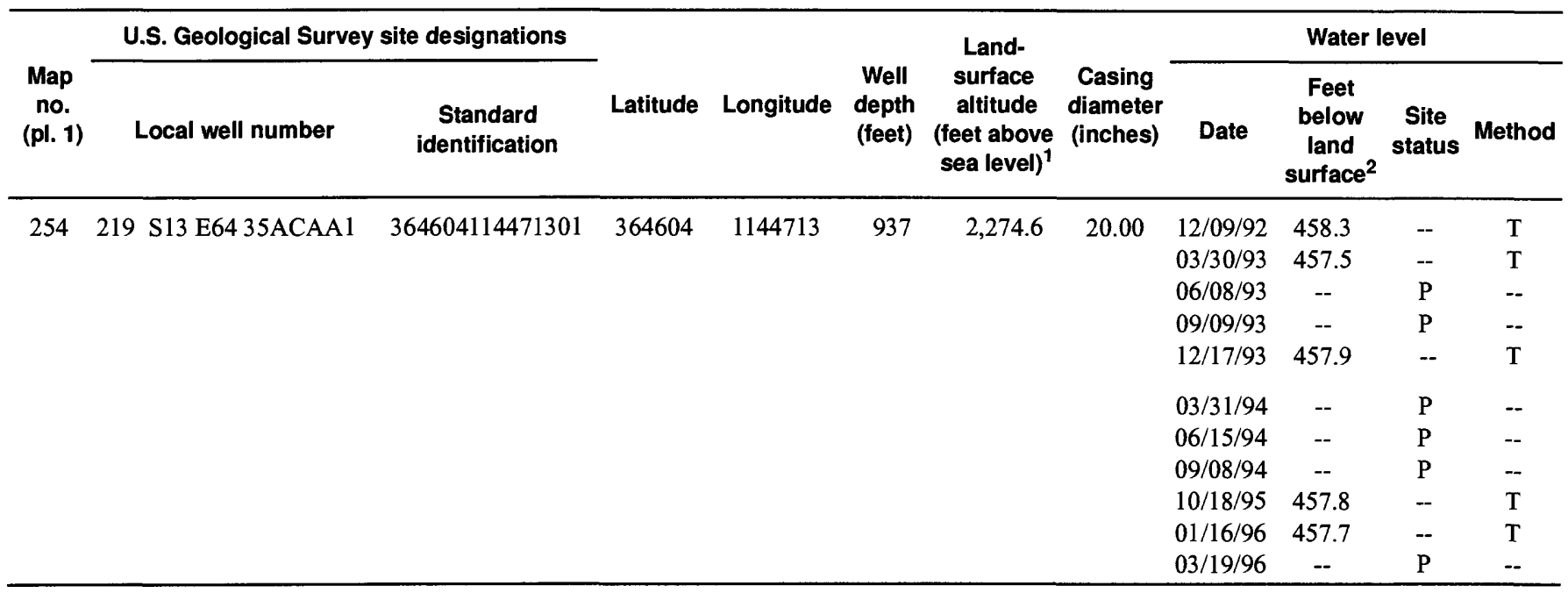

\footnotetext{
${ }^{1}$ Land-surface altitudes recorded to the nearest tenth of a foot have been surveyed from a known reference altitude. All other altitudes are estimated from topographic maps.

${ }^{2}$ Water levels listed herein are from data base. Reported measurements are entered into the data base to the nearest foot; electric-tape measurements are entered to the nearest tenth of a foot; and steel-tape measurements are entered to the nearest hundredth of a foot.
}

Table 3. Monitoring wells with digital recording devices

\begin{tabular}{|c|c|c|c|c|c|c|}
\hline \multirow{2}{*}{$\begin{array}{l}\text { Map } \\
\text { No. } \\
\text { (pl. 1) }\end{array}$} & \multicolumn{2}{|c|}{ U.S. Geological Survey site designations } & \multirow{2}{*}{$\begin{array}{l}\text { Well depth } \\
\text { (feet) }\end{array}$} & \multirow{2}{*}{$\begin{array}{c}\text { Depth to } \\
\text { water }{ }^{1} \\
\text { (feet below } \\
\text { land surface) }\end{array}$} & \multirow{2}{*}{ Recorder } & \multirow{2}{*}{$\begin{array}{c}\text { Date } \\
\text { installed }\end{array}$} \\
\hline & Local well number & $\begin{array}{c}\text { Standard } \\
\text { identification }\end{array}$ & & & & \\
\hline $\begin{array}{l}212 \\
217 \\
219 \\
223 \\
225\end{array}$ & $\begin{array}{l}156 \text { N03 E50 13CA1 } \\
156 \text { N07 E51 10AD1 } \\
171 \text { N03 E59 10BD1 } \\
172 \text { N02 E57 22BBC1 } \\
173 \text { B N03 E52 02DA2 }\end{array}$ & $\begin{array}{l}380652116200901 \\
382901116125201 \\
380758115204601 \\
380132115333501 \\
380906116050502\end{array}$ & $\begin{array}{r}680 \\
480 \\
1,837 \\
1,010 \\
495\end{array}$ & $\begin{array}{l}314.23 \\
236.30 \\
800.00 \\
412.75 \\
231.10\end{array}$ & $\begin{array}{l}\text { TELOG }^{2} \\
\text { TELOG } \\
\text { TUBER }^{3} \\
\text { TELOG } \\
\text { TELOG }\end{array}$ & $\begin{array}{l}09 / 20 / 93 \\
05 / 14 / 92 \\
11 / 20 / 93 \\
09 / 21 / 93 \\
07 / 14 / 93\end{array}$ \\
\hline $\begin{array}{l}229 \\
230 \\
233 \\
234 \\
235\end{array}$ & $\begin{array}{l}\text { 173B N10 E58 17CAAB1 } \\
179 \text { N12 E63 12AB1 } \\
180 \text { N07 E63 14BADD1 } \\
181 \text { N03 E63 27CAA1 } \\
181 \text { N04 E64 07DC1 }\end{array}$ & $\begin{array}{l}384338115283601 \\
385521114503601 \\
382807114521001 \\
380531114534201 \\
381256114500701\end{array}$ & $\begin{array}{r}581 \\
2,447 \\
460 \\
2,395 \\
1,190\end{array}$ & $\begin{array}{l}275.10 \\
428.26 \\
226.80 \\
848.50 \\
254.98\end{array}$ & $\begin{array}{l}\text { TELOG } \\
\text { TELOG } \\
\text { F\&P DIGITAL } \\
\text { TUBER } \\
\text { TELOG }\end{array}$ & $\begin{array}{l}05 / 12 / 92 \\
07 / 19 / 94 \\
10 / 01 / 83 \\
07 / 07 / 95 \\
09 / 21 / 93\end{array}$ \\
\hline $\begin{array}{l}238 \\
244 \\
178 \\
248 \\
252 \\
254\end{array}$ & $\begin{array}{l}181 \text { S03 E64 12AC1 } \\
184 \text { N09 E68 30AAAB1 } \\
195 \text { N11 E70 35AD1 } \\
196 \text { N08 E69 35DC2 } \\
210 \text { S13 E63 23DDDC1 } \\
219 \text { S13 E64 35ACAA1 }\end{array}$ & $\begin{array}{l}374215114453101 \\
383704114225001 \\
384702114041601 \\
383023114115302 \\
364743114533101 \\
364604114471301\end{array}$ & $\begin{array}{r}1,000 \\
679 \\
101 \\
435 \\
669 \\
934\end{array}$ & $\begin{array}{r}394.22 \\
225.85 \\
68.79 \\
173.70 \\
350.90 \\
457.80\end{array}$ & $\begin{array}{l}\text { TELOG } \\
\text { TELOG } \\
\text { TELOG } \\
\text { TUBER } \\
\text { TELOG } \\
\text { TELOG }\end{array}$ & $\begin{array}{l}09 / 21 / 93 \\
05 / 13 / 92 \\
06 / 16 / 93 \\
01 / 19 / 94 \\
09 / 27 / 90 \\
07 / 14 / 94\end{array}$ \\
\hline
\end{tabular}

\footnotetext{
${ }^{1}$ Depth to water when recorder was installed.

2 TELOG is manufactured by Telog Instrument Co., Henrietta, N.Y.

3 TUBER is manufactured by Geoguard Inc., Medina, N.Y.

${ }^{4}$ F\&P DIGITAL is manufactured by Fischer and Porter, Co., Warminster, Penn.

${ }^{5}$ No measurement was made when recorder was installed. Well was being pumped. Depth to water is for October $18,1995$.
} 